\title{
Search for dark matter produced in association with a Standard Model Higgs boson decaying into $b$-quarks using the full Run 2 dataset from the ATLAS detector
}

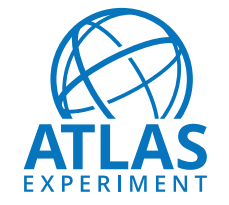

\section{The ATLAS collaboration}

\author{
E-mail: atlas.publications@cern.ch
}

ABSTRACT: The production of dark matter in association with Higgs bosons is predicted in several extensions of the Standard Model. An exploration of such scenarios is presented, considering final states with missing transverse momentum and $b$-tagged jets consistent with a Higgs boson. The analysis uses proton-proton collision data at a centre-of-mass energy of $13 \mathrm{TeV}$ recorded by the ATLAS experiment at the LHC during Run 2, amounting to an integrated luminosity of $139 \mathrm{fb}^{-1}$. The analysis, when compared with previous searches, benefits from a larger dataset, but also has further improvements providing sensitivity to a wider spectrum of signal scenarios. These improvements include both an optimised event selection and advances in the object identification, such as the use of the likelihood-based significance of the missing transverse momentum and variable-radius track-jets. No significant deviation from Standard Model expectations is observed. Limits are set, at 95\% confidence level, in two benchmark models with two Higgs doublets extended by either a heavy vector boson $Z^{\prime}$ or a pseudoscalar singlet $a$ and which both provide a dark matter candidate $\chi$. In the case of the two-Higgs-doublet model with an additional vector boson $Z^{\prime}$, the observed limits extend up to a $Z^{\prime}$ mass of $3 \mathrm{TeV}$ for a mass of $100 \mathrm{GeV}$ for the dark matter candidate. The two-Higgs-doublet model with a dark matter particle mass of $10 \mathrm{GeV}$ and an additional pseudoscalar $a$ is excluded for masses of the $a$ up to $520 \mathrm{GeV}$ and $240 \mathrm{GeV}$ for $\tan \beta=1$ and $\tan \beta=10$ respectively. Limits on the visible cross-sections are set and range from to $0.05 \mathrm{fb}$ to $3.26 \mathrm{fb}$, depending on the missing transverse momentum and $b$-quark jet multiplicity requirements.

KEYWORDS: Dark matter, Hadron-Hadron scattering (experiments)

ArXiv EPrint: 2108.13391 


\section{Contents}

1 Introduction 1

2 ATLAS detector 4

3 Data and simulated event samples 5

4 Object definitions $\quad 6$

5 Event selection $\quad 9$

5.1 Common selections 10

$\begin{array}{ll}5.2 \text { Signal regions } & 10\end{array}$

$\begin{array}{lll}5.2 .1 & \text { Resolved regions } & 10\end{array}$

5.2.2 Merged regions 11

$\begin{array}{lll}5.3 & \text { Background modelling and control regions } & 12\end{array}$

6 Statistical analysis $\quad 13$

$\begin{array}{lll}7 & \text { Systematic uncertainties } & 15\end{array}$

8 Results $\quad 16$

9 Conclusion $\quad 23$

The ATLAS collaboration $\quad 33$

\section{Introduction}

Various astrophysical observations based on gravitational interactions [1, 2] strongly support the existence of dark matter (DM) which interacts through neither the strong nor the electromagnetic force. However, the Standard Model of particle physics (SM) provides no suitable DM candidate particle. There are many complementary search strategies for DM including direct-detection [3-7] and indirect-detection experiments [8] as well as searches at particle colliders.

Since DM particles do not interact electromagnetically or strongly even direct-detection experiments have low efficiencies. Therefore, instead of attempting to detect them directly, any DM particles produced in proton-proton collisions at the Large Hadron Collider [9] (LHC) would be deduced from an imbalance in the transverse momentum measured in that collision event $\left(E_{\mathrm{T}}^{\mathrm{miss}}\right)$. This means that DM particles can only be detected if they are produced in association with visible particles. When there is only one such particle, this gives rise to event topologies referred to as 'mono- $X$ ' final states, where $X$ refers to the visible particle. Prominent examples of these topologies are the mono-jet [10-12], mono- $Z / W[13-15]$ and mono-photon [16-18] final states. 


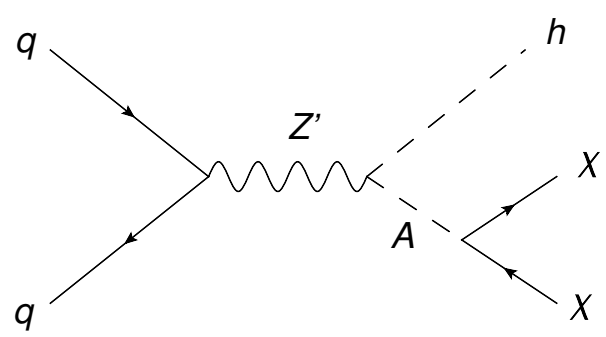

Figure 1. Feynman diagram for the production of the mono-Higgs signature in the $Z^{\prime}-2 H D M$.

Mono- $X$ topologies are typically dominated by cases where the visible particle is produced from initial-state radiation (ISR), as the couplings between the SM particle $X$ and the initial-state quarks or gluons are much larger than its couplings to the final-state DM particles. A counterexample is the case where the visible particle is a SM Higgs boson (named the mono-Higgs signature). Given that the coupling of the Higgs boson to light quarks and gluons is highly suppressed, a Higgs boson is more likely to be produced through final-state radiation (FSR) or as part of the same process that produces the DM particles. This means that this topology is only sensitive to models where the Higgs boson couples directly to DM or some other beyond-the-SM (BSM) particle involved in DM production. However, in these cases the DM-SM interaction is probed directly [19], potentially providing more information about the structure of the DM-SM coupling in the event of a discovery. There are also many models where the coupling between BSM particles and the Higgs boson is enhanced, for instance models where DM is connected to electroweak symmetry breaking $[20,21]$ or where DM particles couple to the SM only through the Higgs sector (Higgs portal models) [22]. These features make the mono-Higgs signature an important part of the LHC DM search programme.

The two-Higgs-doublet model (2HDM) [23] extends the SM with a second Higgs doublet. This predicts a total of five Higgs bosons after mixing: two charged scalars $H^{ \pm}$, two neutral CP-even scalars $h$ and $H$, and one neutral CP-odd scalar $A$. Two simplified benchmark signal models are used: the $Z^{\prime}-2 \mathrm{HDM}[24]$ and $2 \mathrm{HDM}+a[25,26]$. In all models considered here the mass of the lighter neutral CP-even scalar $h$ is required to match that of the Higgs boson observed at the LHC and the Yukawa couplings are defined according to the Type II 2HDM.

The $Z^{\prime}-2 \mathrm{HDM}$ has an additional heavy vector boson, denoted $Z^{\prime}$, whose coupling to quarks $g_{Z^{\prime}}$ and mass $m_{Z^{\prime}}$ are free parameters. The production mechanism for the monoHiggs signature is shown in figure 1. DM is introduced into the model as a new fermion which couples to the CP-odd scalar $A$ with a coupling strength denoted by $g_{\chi}$. The coupling strength and the DM particle mass $m_{\chi}$ are treated as free parameters. This model is used mainly as a benchmark for high-mass resonances.

The $2 \mathrm{HDM}+a$ scenario is the simplest renormalisable and gauge-invariant extension of a simplified pseudoscalar mediator model. It adds a new pseudoscalar singlet which mediates the interactions between the SM and a singlet fermion $\chi$ identified as the DM candidate. The coupling between the pseudoscalar and DM singlets, denoted $y_{\chi}$, and the 


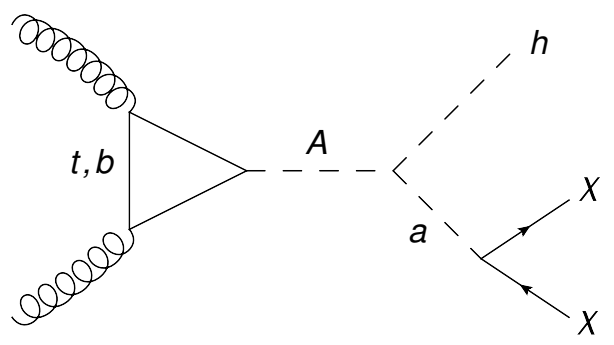

(a)

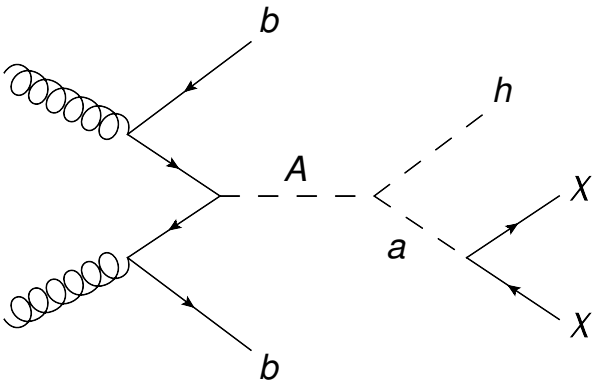

(b)

Figure 2. Feynman diagrams for the main production mechanisms of the mono-Higgs signature in the $2 \mathrm{HDM}+a$ scenario: (a) gluon-gluon fusion and (b) $b$-associated production.

mass of the DM $m_{\chi}$ are free parameters. This singlet mixes with the pseudoscalar $A$ from the two Higgs doublets, with the mixing angle $\theta$ and the mass of the resulting pseudoscalar $a$ being free parameters of the model. A major advantage of the $2 \mathrm{HDM}+a$ scenario over simpler models is that it generates a wider variety of experimental signatures which can provide complementary exclusion regions from different types of experiment. There are two main production mechanisms for the mono-Higgs signature in this model, as shown in figure 2 .

In the type-II $2 \mathrm{HDM}$ considered in this paper the coupling between down-type quarks and the $A$ boson scales with $\tan \beta$, the ratio of the vacuum expectation values of the two Higgs doublets. This means that for low $\tan \beta$ values $(\tan \beta \lesssim 5)$ the gluon-gluon fusion $(g g \mathrm{~F})$ mechanism shown in figure 2(a) dominates, whereas for higher $\tan \beta$ values the $b$ associated production $(b b A)$ shown in figure $2(b)$ is dominant. Signal grids are generated where each of the two production mechanisms are used exclusively. For each grid a $\tan \beta$ value is chosen that ensures that the corresponding production mechanism is dominant: $\tan \beta=1$ for the $g g \mathrm{~F}$ grid and $\tan \beta=10$ for the $b b A$ grid.

Similar analyses were performed using data taken during the years 2015-2016 by ATLAS [27] and CMS [28]. Other mono-Higgs analyses were also performed on the same datasets in final states where the Higgs boson decays into a pair of photons in ATLAS [29] or either a pair of photons, a $\tau^{+} \tau^{-}$pair in CMS [30] or a pair of $W$ or $Z$ bosons [28]. Beyond the large increase in integrated luminosity (from $36 \mathrm{fb}^{-1}$ to $139 \mathrm{fb}^{-1}$ ) a number of analysis improvements extend the sensitivity beyond the previous ATLAS search.

In the previous ATLAS search, events were required to have either one or two $b$-jets, whereas in this search the events are divided into regions with either exactly two or at least three $b$-jets. Introducing the exclusive three $b$-jet category improves the sensitivity to the $2 \mathrm{HDM}+a b b A$ production mechanism which was not considered in the previous ATLAS search, while the one $b$-jet category does not provide any significant improvement due to large backgrounds with high uncertainties.

The analysis also benefits from using particle-flow objects for jet reconstruction [31], neural-network based $b$-jet [32] and $\tau$-lepton [33] identification, and variable-radius trackjets [34] to identify boosted Higgs boson candidates. In order to reduce backgrounds containing fake $E_{\mathrm{T}}^{\mathrm{miss}}$ the likelihood-based $E_{\mathrm{T}}^{\mathrm{miss}}$ significance $\mathcal{S}[35]$ is used. The event selections were reoptimised resulting in an improved sensitivity, especially to highly boosted signals. 


\section{ATLAS detector}

The ATLAS detector [36] at the LHC covers nearly the entire solid angle around the collision point. ${ }^{1}$ It consists of an inner tracking detector surrounded by a thin superconducting solenoid, electromagnetic and hadronic calorimeters, and a muon spectrometer incorporating three large superconducting toroidal magnets.

The inner-detector system (ID) is immersed in a $2 \mathrm{~T}$ axial magnetic field and provides charged-particle tracking in the range $|\eta|<2.5$. The high-granularity silicon pixel detector covers the vertex region and typically provides four measurements per track, the first hit normally being in the insertable B-layer installed before Run 2 [37, 38]. It is followed by the silicon microstrip tracker, which usually provides eight measurements per track. These silicon detectors are complemented by the transition radiation tracker (TRT), which enables radially extended track reconstruction up to $|\eta|=2.0$. The TRT also provides electron identification information based on the fraction of hits (typically 30 in total) above a higher energy-deposit threshold corresponding to transition radiation.

The calorimeter system covers the pseudorapidity range $|\eta|<4.9$. Within the region $|\eta|<3.2$, electromagnetic calorimetry is provided by barrel and endcap high-granularity lead/liquid-argon (LAr) calorimeters, with an additional thin LAr presampler covering $|\eta|<1.8$ to correct for energy loss in material upstream of the calorimeters. Hadronic calorimetry is provided by the steel/scintillator-tile calorimeter, segmented into three barrel structures within $|\eta|<1.7$, and two copper/LAr hadronic endcap calorimeters. The solid angle coverage is completed with forward copper/LAr and tungsten/LAr calorimeter modules optimised for electromagnetic and hadronic measurements, respectively.

The muon spectrometer (MS) comprises separate trigger and high-precision tracking chambers measuring the deflection of muons in a magnetic field generated by the superconducting air-core toroids. The field integral of the toroids ranges between 2.0 and $6.0 \mathrm{Tm}$ across most of the detector. A set of precision chambers covers the region $|\eta|<2.7$ with three layers of monitored drift tubes, complemented by cathode-strip chambers in the forward region, where the background is highest. The muon trigger system covers the range $|\eta|<2.4$ with resistive-plate chambers in the barrel, and thin-gap chambers in the endcap regions.

Interesting events are recorded by the first-level trigger system implemented in custom hardware, followed by selections made by algorithms implemented in software in the highlevel trigger [39]. The first-level trigger reduces the output event rate from the $40 \mathrm{MHz}$ bunch crossing rate to below $100 \mathrm{kHz}$, which the high-level trigger further reduces in order to record events to disk at about $1 \mathrm{kHz}$.

An extensive software suite [40] is used for real and simulated data reconstruction and analysis, for operation and in the trigger and data acquisition systems of the experiment.

\footnotetext{
${ }^{1}$ ATLAS uses a right-handed coordinate system with its origin at the nominal interaction point (IP) in the centre of the detector and the $z$-axis along the beam pipe. The $x$-axis points from the IP to the centre of the LHC ring, and the $y$-axis points upwards. Cylindrical coordinates $(r, \phi)$ are used in the transverse plane, $\phi$ being the azimuthal angle around the $z$-axis. The pseudorapidity is defined in terms of the polar angle $\theta$ as $\eta=-\ln \tan (\theta / 2)$. Angular distance is measured in units of $\Delta R \equiv \sqrt{(\Delta \eta)^{2}+(\Delta \phi)^{2}}$.
} 


\section{Data and simulated event samples}

This search uses $139 \mathrm{fb}^{-1}$ of proton-proton collision data recorded by the ATLAS detector at a centre-of-mass energy of $13 \mathrm{TeV}$ during the years 2015-2018 (Run 2). The uncertainty in the total integrated luminosity for the full Run 2 dataset is $1.7 \%$ [41], obtained using the LUCID-2 detector [42] for the primary luminosity measurements. All events used are required to pass basic data-quality requirements which ensure that all components of the ATLAS detector were functioning correctly [43]. Events selected for the analysis search regions were collected by the primary $E_{\mathrm{T}}^{\text {miss }}$ triggers [44], which select those that have a large transverse momentum imbalance. The algorithms used to calculate the imbalance, and the thresholds required, varied during the data-taking. The 'primary' $E_{\mathrm{T}}^{\text {miss }}$ trigger in a run is the most efficient available $E_{\mathrm{T}}^{\text {miss }}$ trigger, in all cases reaching full efficiency by an offline $E_{\mathrm{T}}^{\text {miss }}$ value of approximately $200 \mathrm{GeV}$.

Simulated event samples corresponding to the $Z^{\prime}-2 \mathrm{HDM}$ signal were generated at leading order (LO) in QCD in the 5-flavour scheme using MADGRAPH5_aMC@NLO v2.6.5 [45] interfaced to Pythia 8.240 [46] using a set of tuned parameters called the A14 tune [47]. Following the recommendations of the ATLAS-CMS Dark Matter Forum [48] the coupling of the $Z^{\prime}$ boson to quarks was fixed to $g_{Z^{\prime}}=0.8$, the mass of the DM candidate was set to $m_{\chi}=100 \mathrm{GeV}$, the $A \chi \bar{\chi}$ coupling $g_{\chi}$ was set to $1, \tan \beta$ was set to 1 , and the alignment limit, i.e. $\sin (\beta-\alpha)=1$, was assumed, where $\alpha$ is the mixing angle between the two CP-even Higgs bosons. The samples were generated with varying $m_{Z^{\prime}}$ between 600 and $3600 \mathrm{GeV}$ and $m_{A}$ between 300 and $1300 \mathrm{GeV}$.

Simulated event samples corresponding to the $2 \mathrm{HDM}+a$ signal were generated at $\mathrm{LO}$ using MadGraph5_aMC@NLO v2.6.7 interfaced to Pythia 8.244 with the A14 tune. Samples were generated separately for the $g g \mathrm{~F}$ and $b b A$ production modes, with the former generated in the 4-flavour scheme setting $\tan \beta=1$ and the latter in the 5 -flavour scheme setting $\tan \beta=10$. Following the recommendations of the LHC Dark Matter Working Group [25], the mass of the DM candidate was set to $m_{\chi}=10 \mathrm{GeV}$, the Yukawa coupling between the DM candidate and the pseudoscalar $a$ was set to $y_{\chi}=1$ and the Higgs quartic couplings were set to $\lambda_{3}=\lambda_{P 1}=\lambda_{P 2}=3$. The chosen value of $m_{\chi}$ ensures that the $a \rightarrow \chi \bar{\chi}$ branching ratio is significant for all values of $m_{a}$ used. The pseudoscalar mixing angle was set to $\sin \theta=0.35$ and the alignment limit was assumed. The samples were generated with varying $m_{A}$ between 250 and $2000 \mathrm{GeV}$ and $m_{a}$ between 100 and $600 \mathrm{GeV}$.

For all signal samples the masses of the heavy Higgs bosons were considered degenerate $\left(m_{A}=m_{H}=m_{H^{ \pm}}\right)$. The mass of the lightest CP-even Higgs boson was set to match that of the Higgs boson discovered at the LHC, i.e. $m_{h}=125 \mathrm{GeV}$ [49].

Background events from the production of a single weak vector boson $(V=W, Z)$ in association with jets or of a pair of weak bosons (diboson) were simulated using Sherpa 2.2.1 [50], with Sherpa 2.2.2 used for $g g$-initiated diboson production. For the $V+$ jets samples, next-to-leading-order (NLO) matrix elements for up to two jets and LO matrix elements for up to four jets were calculated using the Comix [51] and OpenLoops $[52,53]$ libraries. For the $q \bar{q}$-initiated diboson samples, NLO matrix elements for up to one additional jet and LO matrix elements for up to three additional jets were used, 
while the $g g$-initiated processes were generated using LO matrix elements for up to one additional jet. The samples were matched with the SHERPA parton shower [54] using the MEPS@NLO prescription [55-58].

Samples corresponding to the top-quark pair $(t \bar{t})$, single-top-quark (s-, t- and $W t$ channels) and $t \bar{t} h$ processes were generated using PowhEGBox v2 [59-66] with $h_{\text {damp }}$ set to $1.5 m_{\text {top }}$ and $m_{\text {top }}=172.5 \mathrm{GeV}$. The $h_{\text {damp }}$ parameter regulates the transverse momentum $\left(p_{\mathrm{T}}\right)$ of the high- $p_{\mathrm{T}}$ emission against which the $t \bar{t}$ system recoils. The inclusive cross-section for these processes were corrected to next-to-next-to-leading-order (NNLO) plus next-tonext-to-leading-logarithm (NNLL) accuracy for $t \bar{t}$ [67-73], to NLO+NNLL accuracy for $W t[74,75]$, to NLO accuracy for s- and t-channel single top-quark production $[74,75]$ and to NLO QCD+electroweak (EW) accuracy for $t \bar{t} h$ [76]. The diagram removal scheme [77] was used in the $W t$ samples to avoid double counting contributions from $t \bar{t}$ processes.

The $W / Z+h$ samples were generated using PowhegBox v2. The cross-sections of the $q \bar{q}$-initiated processes were calculated at NNLO QCD and NLO EW accuracy, using the Powheg MiNLO procedure [78, 79]. The cross-sections of the $g g$-initiated processes were calculated at NLO+next-to-leading-logarithm (NLL) accuracy in QCD [80-82].

The $t \bar{t} V$ samples were generated using MADGRAPH5_aMC@NLO v2.3.3 at NLO. The cross-sections were calculated at NLO QCD and EW accuracies as provided by ref. [76].

All samples were generated using the NNPDF3.0NLO parton distribution function (PDF) set [83] apart from the SHERPA $W / Z+$ jets and diboson samples, which were generated using the NNPDF3.0NNLO PDF set along with a dedicated tune developed by the SHERPA authors, and the t-channel single-top-quark production samples, which were generated using the NNPDF3.0NLONF4 PDF set. The PowhEGBox samples were interfaced with PYTHIA 8.230 for the parton shower and hadronisation. Of these, the $t \bar{t}$, single-top-quark and $t \bar{t} h$ samples used the NNPDF2.3LO PDF set [83] and the A14 tune [84], while the $W / Z+h$ samples used the CTEQ6L1 PDF set [85] and the AZNLO tune [86]. For the PowhegBox top-quark samples the decays of $b$ - and $c$-hadrons were simulated using EvtGen 1.6.0 [87]. The $t \bar{t} V$ samples were interfaced with PyтніA 8.210 using the same tune and PDF set as the PowhegBox $t \bar{t}$, single-top-quark and $t \bar{t} h$ samples, and using EvTGen 1.2.0 for the decays of $b$ - and $c$-hadrons.

In order to simulate the effect of additional $p p$ collisions in the same and neighbouring bunch crossings (pile-up) all samples were overlaid with multiple $p p$ collisions simulated with Pythia 8.186 using the NNPDF2.3LO PDF set and the A3 tune [88]. The response of the detector was modelled with a detector simulation [89] based on GEANT4 [90].

\section{Object definitions}

Primary vertex. Primary vertices are constructed using at least two ID tracks with $p_{\mathrm{T}}>500 \mathrm{MeV}$ [91]. The primary vertex with the largest sum of squared track transverse momenta $\left(\sum p_{\mathrm{T}}^{2}\right)$ is selected as the hard-scatter vertex, henceforth only referred to as the primary vertex.

Jets. Jets are reconstructed using the anti- $k_{t}$ algorithm $[92,93]$. The analysis considers three types of jets to better match the different event topologies. Small-radius (small- 
$R$ ) jets are constructed from particle-flow objects formed from ID tracks and calorimeter energy clusters [31] using a radius parameter of $R=0.4$. This radius parameter is designed to capture jets initiated by a gluon, light quark or $b$-quark. Small- $R$ jets are classified as central $(|\eta|<2.5)$ or forward $(2.5<|\eta|<4.5)$. Central small- $R$ jets are required to have $p_{\mathrm{T}}>20 \mathrm{GeV}$ and forward small- $R$ jets $p_{\mathrm{T}}>30 \mathrm{GeV}$. In order to remove the impact of jets predominantly formed from particles from pile-up vertices, central small- $R$ jets are required to pass the 'Tight' jet vertex tagger (JVT) [94] working point (WP). ${ }^{2}$

In topologies where the Higgs boson decay $h \rightarrow b \bar{b}$ cannot be resolved into two small- $R$ jets, large-radius (large- $R$ ) jets with a radius parameter of $R=1.0$ are used, constructed from calorimeter energy clusters calibrated using the local hadronic cell weighting (LCW) scheme [95]. This radius parameter is chosen so that a single large- $R$ jet should capture all jets produced in the decay of a boosted heavy object, such as a Higgs boson. To reduce the impact of pile-up, these jets are then 'trimmed', removing any $R=0.2$ subjets which have less than $5 \%$ of the original jet energy [96]. In order to identify subjets originating from $b$-hadrons within the large- $R$ jets, jets are also constructed from ID tracks, using a variant of the anti- $k_{t}$ algorithm with a radius parameter that shrinks as the $p_{\mathrm{T}}$ of the proto-jet increases [34]. These are referred to as variable-radius (variable- $R$ ) track-jets and are matched to the large- $R$ jets by ghost association [97]. The radius parameter is set to $R=30 \mathrm{GeV} / p_{\mathrm{T}}$, with minimum and maximum values of 0.02 and 0.4 , respectively. The reduced radius at high $p_{\mathrm{T}}$ allows the algorithm to reconstruct separate jets from closely spaced $b$-hadrons, such as in highly boosted $h \rightarrow b \bar{b}$ decays.

Both the small- $R$ and large- $R$ jet energies are calibrated using a sequence of simulationderived corrections. Small- $R$ jets additionally have an area-based energy subtraction applied to reduce the impact of pile-up, as well as a series of additional data-derived corrections [98].

Central small- $R$ jets and variable- $R$ track-jets containing $b$-hadrons are identified using the DL1 tagger [32]. This multivariate algorithm uses the impact parameters of ID tracks as well as information about secondary vertices and reconstructed flight paths of $b$ - and $c$-hadrons within the jet. For both classes of jet a WP is chosen which tags jets containing $b$-hadrons with $77 \%$ efficiency in $t \bar{t}$ events. The decays of these $b$-hadrons can produce muons which are vetoed when building particle-flow objects and therefore not included in the energies of either the small- or large- $R$ jets. In order to correct for this, the fourmomenta of non-isolated muons falling inside these jet cones can be added into the jet, improving the resolution of their four-momenta. For small- $R$ (large- $R$ ) jets, this is done for the muon (two muons) closest to the jet axis. This correction is only used when calculating the mass of the Higgs boson candidate $m_{h}$ and was shown in ref. [99] to improve the resolution of this measurement. Correcting $m_{h}$ in this way improves the ability of the fit to separate the signal from the major backgrounds.

Leptons. Leptons are divided into 'baseline' and 'signal' categories. Events with baseline leptons are vetoed in the analysis search regions and signal leptons are used to define control regions to constrain background components.

\footnotetext{
${ }^{2}$ The JVT selection is applied to jets with $20 \mathrm{GeV}<p_{\mathrm{T}}<60 \mathrm{GeV}$ and $|\eta|<2.4$.
} 
Electrons are reconstructed from a track which is coincident with a cluster built from energy deposits in the calorimeter [100]. They are then identified using a multivariate likelihood technique, using several features including the shape of the measured shower, the track quality and the distribution of energy within the calorimeter [101]. For this analysis, the 'LooseAndBLayer' WP [101] is used for both the signal and baseline electrons. Isolation selections are also applied to distinguish between electrons produced in the initial collision or decays of $W / Z$ bosons or $\tau$-leptons (prompt) and those produced in decays of other objects [101]. Requirements are placed on the energy of calorimeter clusters and the $p_{\mathrm{T}}$ of tracks measured in isolation cones around the electron. For signal electrons with $p_{\mathrm{T}}<200 \mathrm{GeV}$ and all baseline electrons the total energy of clusters within $\Delta R=0.2$ of the electron, excluding the electron cluster, must be less than $20 \%$ of the $p_{\mathrm{T}}$ of the electron. The total $p_{\mathrm{T}}$ of tracks matched to the primary vertex that lie within a cone whose size is set to the smaller of $\Delta R=10 \mathrm{GeV} / p_{\mathrm{T}}$ and 0.2 , excluding the electron track, must be less than $15 \%$ of the $p_{\mathrm{T}}$ of the electron. For signal electrons with $p_{\mathrm{T}}>200 \mathrm{GeV}$ the total energy of clusters within $\Delta R=0.2$ of the electron is required to be less than the smaller of $0.015 \times p_{\mathrm{T}}$ and $3.5 \mathrm{GeV}$. For these electrons, no track-based isolation selection is applied. Both the baseline and signal electrons are required to have $|\eta|<2.47$. Baseline electrons are required to have $p_{\mathrm{T}}>7 \mathrm{GeV}$ and signal electrons $p_{\mathrm{T}}>27 \mathrm{GeV}$. In order to ensure that they are compatible with the primary vertex, the track from which the electron is reconstructed is required to have $\sigma\left(d_{0}\right)<5$ and $\left|z_{0} \sin \theta\right|<0.5 \mathrm{~mm}$, where $\sigma\left(d_{0}\right)$ is the significance of the transverse impact parameter, $z_{0}$ is the longitudinal impact parameter, and $\theta$ is the polar angle of the track.

Muons are reconstructed by matching track segments formed in the MS to a track from the ID [102]. Identification is performed through selections on the qualities of the tracks used in the reconstruction, as well as their compatibility, for example, in the measurements of $p_{\mathrm{T}}$ in the MS and ID. Similarly to electrons, isolation selections are also applied. Baseline muons are required to pass the 'Loose' identification WP and signal muons are required to pass the 'Medium' identification WP [102]. For baseline muons, the total energy of clusters within $\Delta R=0.2$ of the muon is required to be less than $30 \%$ of the $p_{\mathrm{T}}$ of the muon. For signal muons, the total $p_{\mathrm{T}}$ of tracks within $\Delta R=0.2$ of the muon's primary track is required to be less than $1.25 \mathrm{GeV}$. Both the baseline and signal muons are required to satisfy $|\eta|<2.5, \sigma\left(d_{0}\right)<3$ and $\left|z_{0} \sin \theta\right|<0.5 \mathrm{~mm}$. Baseline muons are required to have $p_{\mathrm{T}}>7 \mathrm{GeV}$ and signal muons $p_{\mathrm{T}}>25 \mathrm{GeV}$.

Hadronically decaying $\tau$-lepton reconstruction is seeded from $R=0.4$ anti- $k_{t}$ jets built using the LCW-calibrated clusters [103]. As hadronic $\tau$-lepton decays yield either one or three charged pions the jets are required to have either one or three tracks within $\Delta R=0.2$ of the jet axis. A recurrent neural network (RNN) classifier is used to identify the $\tau$-leptons [33]. The inputs to the RNN are built from the clusters and tracks associated with the $\tau$-lepton. All $\tau$-leptons are required to pass the 'VeryLoose' WP [33] and have $|\eta|<2.5$ and $p_{\mathrm{T}}>20 \mathrm{GeV}$. As there is no dedicated $\tau$-lepton control region, no signal $\tau$-lepton selection is defined.

Overlap removal. In order to avoid the same detector signals being interpreted as different objects, an overlap removal procedure is applied as follows. If any object is rejected 
at one step it is not considered in later steps. First, if any two electrons share a track the electron with the lower $p_{\mathrm{T}}$ is removed. Next, any $\tau$-leptons within $\Delta R=0.2$ of an electron or muon are removed. Then, any electrons which share a track with a muon are removed. If any small- $R$ jet is within $\Delta R=0.2$ of an electron it is removed, and then any electron within a cone of $p_{\mathrm{T}}$-dependent size around a small- $R$ jet is removed. If any small- $R$ jet with fewer than three tracks has an associated muon or is within $\Delta R=0.2$ of one it is removed, and then any muons within a cone of $p_{\mathrm{T}}$-dependent size around a small- $R$ jet are removed. Next, any small- $R$ jets within $\Delta R=0.2$ of a $\tau$-lepton are removed. Finally, any large- $R$ jets within $\Delta R=1.0$ of an electron are removed.

Track-jets do not participate in the overlap removal as they are only used for $b$-tagging.

Missing transverse momentum. The missing transverse momentum (with magnitude $E_{\mathrm{T}}^{\mathrm{miss}}$ [104]) is defined as the negative vector sum of the transverse momenta of all the observable objects in the event, plus a soft term including ID tracks matched to the primary vertex but not to any of the other objects. The $E_{\mathrm{T}}^{\text {miss }}$ reconstruction uses the baseline electrons and muons as well as all small- $R$ jets, and employs a separate overlap removal procedure which takes into account detector signals from each object included [104]. In control regions a modified definition of $E_{\mathrm{T}}^{\text {miss }}$ is used in which electrons and muons are treated as invisible, $E_{\mathrm{T} \text {, lep. invis. }}^{\text {miss }}$ to imitate the kinematics of the $Z \rightarrow \nu \bar{\nu}$ background process.

Object mismeasurements, especially of jets, are the main source of fake $E_{\mathrm{T}}^{\text {miss }}$. Therefore, the $E_{\mathrm{T}}^{\text {miss }}$ significance $(\mathcal{S})[35]$ is defined to assess the likelihood that the $E_{\mathrm{T}}^{\text {miss }}$ is really due to invisible particles or is more likely to come from mismeasurements. It is calculated using the expected resolutions of all objects which enter the $E_{\mathrm{T}}^{\text {miss }}$ calculation and the correlations between them.

\section{Event selection}

The basic target final-state topology is a Higgs boson decaying into two $b$-quarks produced with a significant imbalance in the measured transverse momentum. Events are divided into non-overlapping regions designed either to be enriched in the signal process (signal regions) or in a significant background process (control regions). Control regions differ from signal regions primarily through requiring the presence of one or two lepton(s), whereas signal regions veto events containing baseline leptons.

As the angle between the two $b$-jets produced in the Higgs boson decay is inversely proportional to the $p_{\mathrm{T}}$ of the Higgs boson, in cases where the Higgs boson is significantly boosted it can become difficult to reconstruct the two $b$-quarks as separate jets. This motivates splitting the analysis into 'resolved' regions in which the decay products of the Higgs boson are reconstructed as two separate jets and 'merged' regions in which the entire Higgs boson decay is reconstructed as a single jet.

In $b$-associated production within the $2 \mathrm{HDM}+a$ benchmark model, the Higgs boson and DM particles are produced with an extra pair of $b$-quarks from gluon splitting. Therefore, to enhance sensitivity to these models, all regions are further split into those requiring exactly two $b$-jets and those requiring $\geq 3 b$-jets (referred to as $2 b$-tag and $\geq 3 b$-tag, respectively). 


\subsection{Common selections}

Events which do not have a reconstructed primary vertex are rejected. Events are also rejected if found to contain any jets with properties consistent with beam-induced backgrounds, cosmic-ray showers or noisy calorimeter cells [105].

Events are required to have $E_{\mathrm{T}}^{\text {miss }}>150 \mathrm{GeV}$ and are vetoed if they contain a baseline $\tau$-lepton. In order to further reduce the background from $\tau$-lepton decays, events are also vetoed if they have any small- $R$ jets with $\Delta \phi\left(\right.$ jet, $\left.E_{\mathrm{T}}^{\text {miss }}\right)<22.5^{\circ}$ where the track multiplicity in the jet is between 1 and 4 . This selection is referred to as the 'extended $\tau$-lepton veto'. A further source of background is $E_{\mathrm{T}}^{\text {miss }}$ arising from either leptonic heavyflavour decays in a jet or a jet which is severely mismeasured. In these cases, the $E_{\mathrm{T}}^{\text {miss }}$ tends to be aligned with the jet; therefore, events where any of the up to three leading small- $R$ jets have $\Delta \phi\left(\right.$ jet, $\left.E_{\mathrm{T}}^{\mathrm{miss}}\right)<20^{\circ}$ are rejected. For control regions, these requirements use $E_{\mathrm{T} \text {, lep. invis. }}^{\text {miss }}$ rather than $E_{\mathrm{T}}^{\mathrm{miss}}$.

Only loose selections are placed on the mass of the Higgs boson candidate $\left(m_{h}\right.$, defined in the following sections) because it is used as the discriminating variable for the final fit. The range is $50 \mathrm{GeV}<m_{h}<280 \mathrm{GeV}$ for the resolved regions and $50 \mathrm{GeV}<m_{h}<270 \mathrm{GeV}$ for the merged regions, with the lower limit chosen to be the lowest calibrated large- $R$ jet mass and the upper limit chosen to be significantly larger than the Higgs boson mass, with the precise value being determined by the $m_{h}$ binning used in the fit, which depends on the available sample size.

\subsection{Signal regions}

The signal region selections for both the merged and resolved regions are summarised in table 1. All signal region events are required to have passed the primary $E_{\mathrm{T}}^{\text {miss }}$ trigger [44]. In order to reduce the contribution of SM processes producing $E_{\mathrm{T}}^{\text {miss }}$ through the decay $W \rightarrow \ell \nu$, events are rejected if they contain any baseline electron or muon.

\subsubsection{Resolved regions}

The resolved regions are defined by selecting events with $E_{\mathrm{T}}^{\text {miss }}<500 \mathrm{GeV}$. Events in the resolved regions are required to have at least two $b$-tagged small- $R$ jets, with the two with the highest $p_{\mathrm{T}}$ forming the Higgs boson candidate. The combined $p_{\mathrm{T}}$ of this two-jet system $\left(p_{\mathrm{T}_{h}}\right)$ is required to be greater than $100 \mathrm{GeV}$ and its mass is corrected for nearby muons as described in section 4 to form $m_{h}$.

The dominant background in the resolved region is $t \bar{t}$ production where one top quark decays leptonically, but the lepton is either not reconstructed or not correctly identified. In these cases, all the $E_{\mathrm{T}}^{\mathrm{miss}}$ in the event (beyond that from mismeasurement) originates from the decay of one of the two $W$ bosons, and therefore the transverse mass of the $E_{\mathrm{T}}^{\text {miss }}$ and the corresponding $b$-jet should be approximately bounded from above by the top-quark mass. Here the transverse mass is defined as

$$
m_{\mathrm{T}}^{b, \min / \max }=\sqrt{2 p_{\mathrm{T}}^{b, \min / \max } E_{\mathrm{T}}^{\mathrm{miss}}\left(1-\cos \Delta \phi\left(p_{\mathrm{T}}^{b, \min / \max }, E_{\mathrm{T}}^{\mathrm{miss}}\right)\right)}
$$


where $p_{\mathrm{T}}^{b, \min }$ and $p_{\mathrm{T}}^{b, \max }$ are respectively defined as the $p_{\mathrm{T}}$ of the $b$-jet which is closest to $(\min )$ or furthest from $(\max )$ the $E_{\mathrm{T}}^{\text {miss }}$ in $\phi$. Events are required to satisfy $m_{\mathrm{T}}^{b, \min }>$ $170 \mathrm{GeV}$ and $m_{\mathrm{T}}^{b, \max }>200 \mathrm{GeV}$.

In order to suppress contributions from multijet backgrounds, the object-based $E_{\mathrm{T}}^{\mathrm{miss}}$ significance is required to satisfy $\mathcal{S}>12$. After this selection, data-driven estimates of the remaining multijet contribution to the signal regions were found to be substantially smaller than the expected statistical uncertainty of the data so the impact of multijet processes is not included in the background estimation. The studied signal models typically have fewer reconstructed jets than the dominant backgrounds. Therefore, events with exactly two $b$-tagged jets ( $2 b$-tag) are required to have at most four small- $R$ jets, where only central jets are counted. For events with at least three $b$-tagged jets ( $\geq 3 b$-tag), this requirement is relaxed to at most five small- $R$ jets to ensure a sufficient sample size in the corresponding control regions.

The $2 b$-tag and $\geq 3 b$-tag selections are split into three $E_{\mathrm{T}}^{\text {miss }}$ bins: $150 \mathrm{GeV}<E_{\mathrm{T}}^{\text {miss }}<$ $200 \mathrm{GeV}, 200 \mathrm{GeV}<E_{\mathrm{T}}^{\text {miss }}<350 \mathrm{GeV}$ and $350 \mathrm{GeV}<E_{\mathrm{T}}^{\text {miss }}<500 \mathrm{GeV}$. In the highest $E_{\mathrm{T}}^{\text {miss }}$ bin the requirement on $p_{\mathrm{T} h}$ is tightened to being greater than $300 \mathrm{GeV}$. This leads to six resolved signal regions: one corresponding to each combination of $E_{\mathrm{T}}^{\text {miss }}$ bin and number of $b$-tagged jets.

The $E_{\mathrm{T}}^{\text {miss }}$ triggers become fully efficient at an offline $E_{\mathrm{T}}^{\text {miss }}$ value close to $200 \mathrm{GeV}$, but the analysis also uses events in the range $150 \mathrm{GeV}<E_{\mathrm{T}}^{\text {miss }}<200 \mathrm{GeV}$. In order to correct for Monte Carlo (MC) mismodelling of the $E_{\mathrm{T}}^{\text {miss }}$ trigger response, the trigger efficiency must be measured in both data and simulation and scale factors calculated to correct the simulation. Given that the $E_{\mathrm{T}}^{\text {miss }}$ triggers use calorimeter information only, muons are treated as almost invisible particles, meaning that $E_{\mathrm{T}}^{\text {miss }}$ trigger efficiencies can be measured using events selected by single-muon triggers [106]. The scale factors are calculated as a function of $E_{\mathrm{T}}^{\text {miss }}$ lep, invis in a region whose selection matches the $150 \mathrm{GeV}<E_{\mathrm{T}}^{\text {miss }}<200 \mathrm{GeV}$

and $2 b$-tag region except that all $E_{\mathrm{T}}^{\text {miss }}$ selections are dropped, exactly one $b$-tagged jet is required and exactly one signal muon is required. Events containing electrons are still vetoed. The scale factors have values in the range $0.95-1.0$.

\subsubsection{Merged regions}

The merged regions are defined by selecting events with $E_{\mathrm{T}}^{\text {miss }}>500 \mathrm{GeV}$. At least one large- $R$ jet is required, and the two leading variable- $R$ track-jets associated with the leading large- $R$ jet are required to be $b$-tagged. This large- $R$ jet is defined to be the Higgs boson candidate and its mass is corrected for nearby muons as described in section 4 to form $m_{h}$. Events are separated into those which have no additional $b$-tagged variable- $R$ track-jets ( $2 b$-tag selection) and those which have at least one such $b$-tagged variable- $R$ track-jet not associated with the Higgs boson candidate ( $\geq 3$ b-tag selection).

The merged $2 b$-tag selection is split into two $E_{\mathrm{T}}^{\text {miss }}$ bins, $500 \mathrm{GeV}<E_{\mathrm{T}}^{\text {miss }}<750 \mathrm{GeV}$ and $E_{\mathrm{T}}^{\text {miss }}>750 \mathrm{GeV}$, while no further splitting is done for the $\geq 3 b$-tag selection. 


\begin{tabular}{|c|c|}
\hline \multicolumn{1}{|c|}{ Resolved } & Merged \\
\hline \multicolumn{2}{|c|}{ Primary $E_{\mathrm{T}}^{\text {miss }}$ trigger } \\
\hline \multicolumn{2}{|c|}{$E_{\mathrm{T}}^{\text {miss }}>150 \mathrm{GeV}$} \\
\hline \multicolumn{2}{|c|}{ Lepton veto \& extended $\tau$-lepton veto } \\
\hline \multicolumn{2}{|c|}{$\Delta \phi\left(\right.$ jet $\left._{1,2,3}, E_{\mathrm{T}}^{\text {miss }}\right)>20^{\circ}$} \\
\hline \multicolumn{2}{|c|}{$E_{\mathrm{T}}^{\text {miss }}>500 \mathrm{GeV}$} \\
\hline$E_{\mathrm{T}}^{\text {miss }}<500 \mathrm{GeV}$ & At least 1 large- $R$ jet \\
\hline At least 2 small- $R$ jets & - \\
\hline At least $2 b$-tagged small- $R$ jets & At least $2 b$-tagged associated variable- $R$ track-jets \\
\hline$p_{\mathrm{T} h}>100 \mathrm{GeV}$ if $E_{\mathrm{T}}^{\text {miss }}<350 \mathrm{GeV}$ & - \\
$p_{\mathrm{T} h}>300 \mathrm{GeV}$ if $E_{\mathrm{T}}^{\text {miss }}>350 \mathrm{GeV}$ & - \\
\hline$m_{\mathrm{T}}^{b, \text { min }}>170 \mathrm{GeV}$ & - \\
\hline$m_{\mathrm{T}}^{b, \text { max }}>200 \mathrm{GeV}$ & \\
\hline $\mathcal{S}>12$ & $50 \mathrm{GeV}<m_{h}<270 \mathrm{GeV}$ \\
\hline$N_{\text {small- } R \text { jets }} \leq 4$ if $2 b$-tag & - \\
$N_{\text {small- } R \text { jets }} \leq 5$ if $\geq 3 b$-tag & \\
\hline $50 \mathrm{GeV}<m_{h}<280 \mathrm{GeV}$ & \\
\hline
\end{tabular}

Table 1. Summary of selections used to define the signal regions used in the analysis. The kinematic variables are defined in the text.

\subsection{Background modelling and control regions}

The dominant backgrounds in the signal regions consist of $t \bar{t}$ and $W / Z$ bosons produced in association with heavy-flavour jets. The $W / Z+$ jets backgrounds are subdivided according to the true flavour of the jets that constitute the Higgs boson candidate. ${ }^{3}$ If the flavour of either (or both) those two jets is a $b$-quark, the event is considered to be $W / Z+\mathrm{HF}$ background, where HF stands for Heavy Flavour. In the $2 b$-tag resolved regions the dominant backgrounds are $t \bar{t}$ and $Z+\mathrm{HF}$, with the latter becoming more important as the $E_{\mathrm{T}}^{\text {miss }}$ increases. The $2 b$-tag merged regions are dominated by $Z+\mathrm{HF}$. Both the resolved and merged $\geq 3 b$-tag regions are dominated by $t \bar{t}$, where the extra $b$-jet typically is a mistagged jet originating from a hadronic $W$ boson decay. At higher $E_{\mathrm{T}}^{\text {miss }}$ values the $Z+\mathrm{HF}$ background becomes important again.

The $t \bar{t}, W+\mathrm{HF}$ and $Z+\mathrm{HF}$ contributions are modelled using simulation with their normalisations corrected from data by using background-enriched control regions besides the signal regions. Smaller backgrounds are taken directly from simulation. These include the production of a $W$ or $Z$ boson in association with light jets or at most one jet containing a $c$-hadron, single top-quark production (dominated by production in association with a $W$

\footnotetext{
${ }^{3}$ Simulated jets are labelled according to which hadrons with $p_{\mathrm{T}}>5 \mathrm{GeV}$ are found within a cone of size $\Delta R=0.3$ around the jet axis. If a $b$-hadron is found the jet is labelled as a $b$-jet. If no $b$-hadron is found, but a $c$-hadron is present, then the jet is labelled as a $c$-jet. Otherwise the jet is labelled as a light jet. The flavour of the two leading $b$-tagged track-jets is used in the merged region.
} 
boson) and diboson processes. Small contributions also arise from $t \bar{t}$ processes in association with vector bosons or a Higgs boson. Another background contribution stems from vectorboson production in association with a Higgs boson $(V h)$, which mimics the signal due to the presence of a Higgs boson peak in association with jets. In the case where the vector boson is a $Z$ boson decaying to neutrinos this is an irreducible background. Similarly, the diboson decay $Z Z \rightarrow b \bar{b} \nu \nu$ is nearly irreducible due to small difference in $Z$ and $h$ mass peaks (compared to the Higgs candidate mass resolution). The multijet background is negligible in all regions after the requirements on the object-based $E_{\mathrm{T}}^{\mathrm{miss}}$ significance $S$ and on $\Delta \phi\left(\right.$ jet, $\left.E_{\mathrm{T}}^{\mathrm{miss}}\right)$, and thus not further considered. The total background estimates in all regions, including uncertainties, are determined in a simultaneous fit to all regions, which is described in section 6 .

Top-quark pair production and $W+\mathrm{HF}$ processes contribute in the signal regions if leptons in the decays are either not identified or outside the kinematic acceptance. The main contribution arises from decays involving hadronically decaying $\tau$-leptons. As the shape of the event variables is the same for all lepton flavours, the kinematic phase space of the signal region can be closely approximated by control regions requiring an isolated signal muon (1-muon control regions). In this case, to better approximate the signal regions

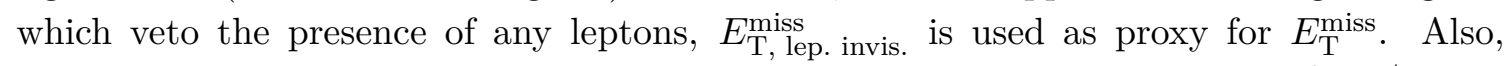
any other variable using $E_{\mathrm{T}}^{\mathrm{miss}}$ in its calculation, e.g. $E_{\mathrm{T}}^{\mathrm{miss}}$ significance and $m_{\mathrm{T}}^{\mathrm{b}, \min / \mathrm{max}}$, is constructed using $E_{\mathrm{T} \text {, lep. invis. }}^{\text {miss }}$ This ensures that the $E_{\mathrm{T}}^{\text {miss }}$-related quantities in the control regions correspond to those in the signal regions. Otherwise, the 1-muon control regions are defined by the same criteria as the signal regions.

As the momentum of the $Z$ boson does not depend on its decay mode, the main background in the signal regions, $Z \rightarrow \nu \nu$ in association with heavy-flavour jets, can be closely modelled by $Z \rightarrow \ell^{+} \ell^{-}$events. This means that the normalisation of $Z \rightarrow \nu \nu+\mathrm{HF}$ contribution can be corrected by measuring $Z \rightarrow \ell^{+} \ell^{-}+$HF events. To select these events, control regions requiring exactly two baseline electrons or muons with opposite charge are defined (2-lepton control regions). These events are collected using primary triggers selecting an isolated electron or muon [106, 107]. Further, one of the electrons or muons is required to be a signal electron or muon with $p_{\mathrm{T}}>27 \mathrm{GeV}$ or $p_{\mathrm{T}}>25 \mathrm{GeV}$, respectively. The invariant mass of the leptons is required to be consistent with the mass of the $Z$ boson within $10 \mathrm{GeV}$. While keeping all other criteria of the signal regions, an additional criterion of $S<5$ is imposed to suppress a remaining contribution of $t \bar{t}$ processes. To be similar to the signal regions, $E_{\mathrm{T}}^{\text {miss }}$ lep. invis. is used as proxy for $E_{\mathrm{T}}^{\text {miss }}$ and in the calculation of any other variable using $E_{\mathrm{T}}^{\mathrm{miss}}$.

\section{$6 \quad$ Statistical analysis}

A binned profile likelihood fit $[108,109]$ is used to obtain background estimates and check the compatibility of the data with the background-only hypothesis as well as to extract upper limits at $95 \%$ confidence level (CL) on the signal cross-section. The likelihood function is constructed from a product of Poisson probability functions based on the expected signal and background yields in every region considered in the fit, as shown in table 2. It contains 


\begin{tabular}{|c|c|c|c|}
\hline & 0 lepton & 1 muon & 2 leptons \\
\hline Aim & Signal regions & $t \bar{t}$ and $W+\mathrm{HF}$ control region & $Z+\mathrm{HF}$ control regions \\
\hline Fitted observable & $m_{h}$ distribution & $\begin{array}{l}\text { Muon charge }(2 \text { b-tag }) \\
\text { Yields }(\geq 3 b \text {-tag })\end{array}$ & Yields \\
\hline$b$-tag multiplicities & \multicolumn{3}{|c|}{$\begin{array}{l}\text { resolved (small- } R \text { jets): } 2, \geq 3 \\
\text { merged (variable- } R \text { track-jets): } \\
\text { e } h \text { candidate), } \geq 3 \text { ( } 2 \text { inside } h \text { candidate })\end{array}$} \\
\hline$E_{\mathrm{T}}^{\text {miss }}$ proxy & $E_{\mathrm{T}}^{\mathrm{miss}}$ & $E_{\mathrm{T}, \text { lep. invis. }}^{\text {miss }}$ & $E_{\mathrm{T}, \text { lep. invis. }}^{\text {miss }}$ \\
\hline Bins in $E_{\mathrm{T}}^{\text {miss }}$ proxy & \multicolumn{3}{|c|}{$\begin{array}{c}2 b \text {-tag merged signal regions (0 lepton): }[500,750) \text { and }[750, \infty) \mathrm{GeV} \\
\text { Other merged regions: }[500, \infty) \mathrm{GeV}\end{array}$} \\
\hline
\end{tabular}

Table 2. Event categories entering the combined fit of the model to the data. The discriminant $m_{h}$ denotes the mass of the light Higgs boson candidate and corresponds either to the dijet mass $m_{j j}$ in the regions selecting two small- $R$ jets or to the large- $R$ jet mass $m_{J}$ for the regions requiring a large- $R$ jet. "Yields" refers to the number of events in a given region.

the parameter of interest, $\mu$, which multiplies the signal cross-section, as well as floating normalisation factors controlling the background normalisations. Systematic uncertainties are included in the likelihood by nuisance parameters (NP) $\theta$ which are parameterised by Gaussian or log-normal priors.

Four normalisation factors are used for the backgrounds, scaling the $t \bar{t}$ and $W / Z+\mathrm{HF}$ processes. Of those, two normalisation factors are used for $Z$ boson production in association with two $b$-tagged jets or at least three $b$-tagged jets. In the $2 b$-tag and $\geq 3 b$-tag selections, $t \bar{t}$ and $W+\mathrm{HF}$ are normalised by a single parameter each, as the production mechanism for $t \bar{t}$ is the same in the two categories and the contribution of $W+\mathrm{HF}$ is minor in the $\geq 3 b$-tag selection.

The likelihood includes all control and signal regions, as detailed in table 2, where these regions are binned to increase the sensitivity and improve the determination of the normalisation factors for $t \bar{t}$ and $W / Z+\mathrm{HF}$. The signal regions are binned in the invariant mass of the Higgs boson candidate as defined in section 5. The chosen binning in the signal regions is optimised to obtain the best expected sensitivity to the signal models addressed in this paper, while also keeping statistical uncertainties in each bin low.

The 1-muon control regions are split in positive or negative muon charge in the case of the $2 b$-tag regions. This improves the separation between $t \bar{t}$ and $W+\mathrm{HF}$ processes, as $W+\mathrm{HF}$ processes exhibit a charge asymmetry in $p p$ collisions. Only inclusive event yields are used in the other regions, which are dominated by $t \bar{t}$, and for the 2-lepton control regions.

The nominal fit results are obtained by maximising the likelihood function with respect to all parameters. Two different fit configurations are used: in the background-only profile likelihood fit, the parameters are determined in a fit to data assuming the presence of no 
signal. The second fit configuration instead allows for the presence of a specific signal, and is referred to as the model-dependent fit.

The test statistic $q_{\mu}$ is constructed using the profile likelihood [110]: $q_{\mu}=$ $-2 \ln \left(\mathcal{L}\left(\mu, \hat{\theta_{\mu}}\right) / \mathcal{L}(\hat{\mu}, \hat{\theta})\right)$, where $\hat{\mu}$ and $\hat{\theta}$ are the parameters that maximise the likelihood, and $\hat{\hat{\theta}}_{\mu}$ are the nuisance parameter values that maximise the likelihood for a given $\mu$. This test statistic is used to measure the compatibility of the background-only model with the observed data and to derive exclusion intervals using the $\mathrm{CL}_{s}$-prescription [111].

\section{Systematic uncertainties}

Signal and background expectations are subject to statistical, detector-related and theoretical uncertainties, which are all included in the likelihood as nuisance parameters. Detector-related and theoretical uncertainties may affect the overall normalisation and/or shape of the simulated background and signal event distributions.

Detector-related uncertainties are dominated by contributions from the jet reconstruction. Uncertainties in the jet energy scale (JES) for small- $R$ jets [98] arise from the calibration of the scale of the jet and are derived as function of the jet $p_{\mathrm{T}}$, and also $\eta$. Further contributions emerge from the jet flavour composition and the pile-up conditions. The 'category reduction' scheme as described in ref. [98] with 29 nuisance parameters is used. Uncertainties in the jet energy resolution (JER) depend on the jet $p_{\mathrm{T}}$ and $\eta$ and arise both from the method used to derive the jet resolution and from the difference between simulation and data [98], and they are included with eight nuisance parameters. Similarly, uncertainties in the jet energy resolution for large- $R$ jets arise from the calibration, the flavour composition and the topology dependence [112]. Further uncertainties are considered for the large- $R$ jet mass scale [112] and resolution [113].

Uncertainties due to the $b$-tagging efficiency for heavy-flavour jets, including $c$-flavour jets, are derived from $t \bar{t}$ data $[114,115]$ and are represented by four nuisance parameters. Uncertainties are also considered for mistakenly $b$-tagging a light-flavour jet, with nine nuisance parameters. These are estimated using a method similar to that in ref. [116].

Uncertainties in the modelling of $E_{\mathrm{T}}^{\mathrm{miss}}$ are evaluated by considering the uncertainties affecting the jets included in the calculation and the uncertainties in soft term's scale and resolution [117]. The pile-up in simulation is matched to the conditions in data by a reweighting factor. An uncertainty of $4 \%$ is assigned to this reweighting factor. The uncertainty in the combined 2015-2018 integrated luminosity is $1.7 \%$ [41], obtained using the LUCID-2 detector [42] for the primary luminosity measurements.

Scale factors, including their uncertainties, are calculated specifically for this analysis to correct the efficiency of $E_{\mathrm{T}}^{\text {miss }}$ triggers in simulation to that in data. The uncertainties in the scale factors are at most $1 \%-2 \%$ for low $E_{\mathrm{T}}^{\text {miss }}$ values.

In the regions requiring the presence of leptons, uncertainties in the lepton identification and lepton energy/momentum scale and resolution are included. These are derived using simulated and measured events with $Z \rightarrow \ell^{+} \ell^{-}, J / \psi \rightarrow \ell^{+} \ell^{-}$and $W \rightarrow \ell \nu$ decays $[100,102]$. 
Modelling uncertainties impact the shape of the $m_{h}$ distribution, the relative acceptance between different $E_{\mathrm{T}}^{\text {miss }}$ and $b$-tag multiplicity bins and between signal and control regions as well as the overall normalisation of the samples that are not freely floating in the fit.

The theoretical uncertainties are dominated by modelling uncertainties in the $t \bar{t}$ and $Z+\mathrm{HF}$ backgrounds. For the $t \bar{t}$ and $W t$ processes, the impact of the choice of parton shower and hadronisation model is evaluated by comparing the sample from the nominal generator set-up with a sample interfaced to HeRwig $7.04[118,119]$. To assess the uncertainty in the matching of NLO matrix elements to the parton shower, the PowHEGBox sample is compared with a sample of events generated with MADGRAPH5_aMC@NLO v2.6.2. For the $W t$ process, the nominal sample is compared with an alternative sample generated using the diagram subtraction scheme $[77,84]$ instead of the diagram removal scheme to estimate the uncertainty arising from the interference with $t \bar{t}$ production.

For the $V+$ jet processes, uncertainties arising from the modelling of the parton shower and the matching scheme are evaluated by comparing the nominal samples with samples generated with MADGRAPH5_aMC@NLO v2.2.2. For the diboson processes, the uncertainties associated with the modelling of the parton shower, the hadronisation and the underlying event are derived using alternative samples generated with PowHEGBox [6062] and interfaced to Pythia 8.186 [120] or Herwig++.

For all MC samples, the uncertainties due to missing higher orders are estimated by a variation of the renormalisation and factorisation scales by a factor of two, while the PDF and $\alpha_{\mathrm{s}}$ uncertainties are calculated using the PDF4LHC prescription [121].

Table 3 gives the impact of the different sources of systematic uncertainties for selected signal models as evaluated in different model-dependent fits. The signal models with lower masses illustrate the impact of the systematic uncertainties in the resolved regions, while the models with larger mediator masses are more impacted by the merged regions. The theoretical uncertainties in the modelling of the $t \bar{t}$ background, the experimental uncertainties in the calibration of jets and the limited MC sample size show the largest impact.

\section{Results}

The post-fit background yields are determined in a background-only profile likelihood fit to data in all regions. Figure 3 shows the yields in the 1-muon and 2-lepton control regions. The post-fit normalisation factors for $t \bar{t}$ and for $W+\mathrm{HF}$ are found to be $0.93 \pm 0.08$ and $0.95 \pm 0.14$, respectively. For $Z$ boson production in association with two (at least three) heavy-flavour jets the normalisation factors are determined to $1.41 \pm 0.09(1.85 \pm 0.24)$. An upward scaling of the $Z+\mathrm{HF}$ background relative to the simulation was also observed in other studies [122], and was attributed to an underestimation of the $g \rightarrow b \bar{b}$ rate in SHERPA. A larger scaling is observed in the region with $\geq 3 b$-tagged jets, dominated by processes with more $g \rightarrow b \bar{b}$ splittings. The uncertainty on the $Z+$ HF normalisation factor increases in the $\geq 3 b$-tag region due to lower statistical precision and the smaller contribution of the $Z+\mathrm{HF}$ background. 


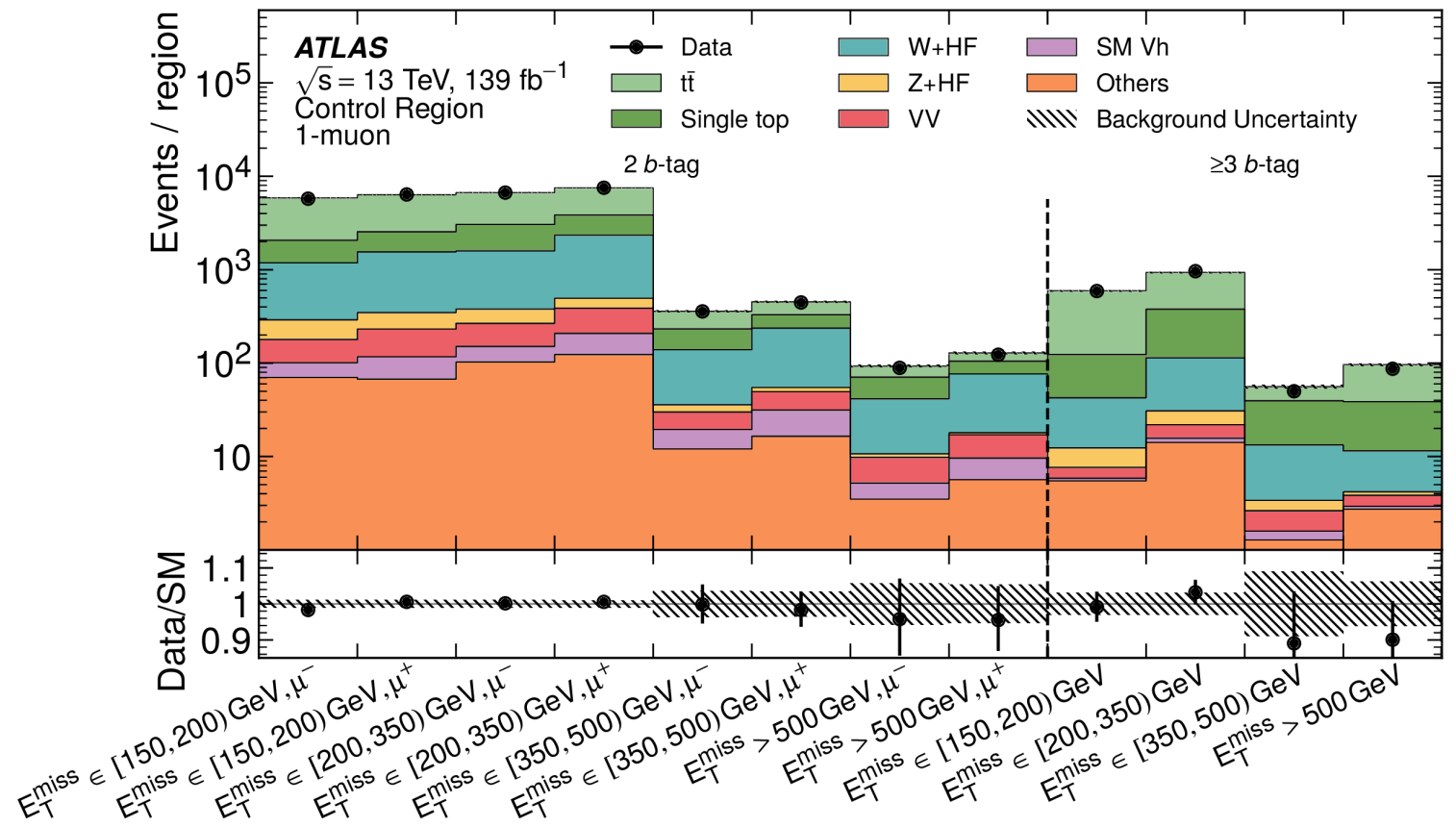

(a)

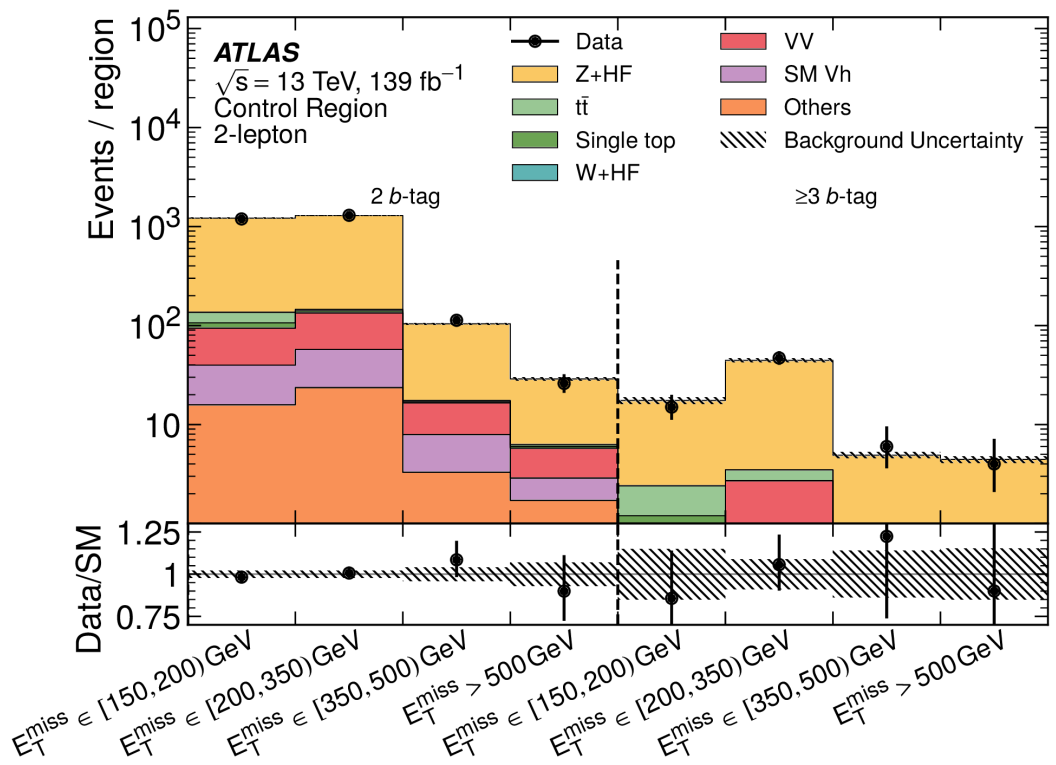

(b)

Figure 3. Yields in the resolved and merged (a) 1-muon control regions and (b) 2-lepton control regions. The top panel compares the fitted background yields with data, while the bottom panel indicates the ratio of the observed data to the predicted Standard Model backgrounds. The different control region bins included in the fit are indicated on the $x$-axis by first giving the range in $E_{\mathrm{T}}^{\text {miss }}$ and then the sign of the muon charge (where applicable). 


\begin{tabular}{|l|ccc|}
\hline \multirow{3}{*}{ Source of uncertainty } & \multicolumn{3}{|c|}{ Fractional squared uncertainty in $\mu$} \\
\cline { 2 - 4 } & \multicolumn{3}{|c|}{$Z^{\prime}$-2HDM signals, $\left(m_{Z}^{\prime}, m_{A}\right)[\mathrm{GeV}]$} \\
& $(800,500)$ & $(1400,1000)$ & $(2800,300)$ \\
\hline$Z+\mathrm{HF}$ normalisation & 0.11 & 0.03 & $<0.01$ \\
$W+\mathrm{HF}$ normalisation & 0.02 & 0.01 & $<0.01$ \\
$t \bar{t}$ normalisation & 0.16 & 0.04 & $<0.01$ \\
$Z$ modelling uncertainties & 0.02 & 0.07 & $<0.01$ \\
$W$ modelling uncertainties & $<0.01$ & 0.01 & $<0.01$ \\
$t \bar{t}$ modelling uncertainties & 0.13 & 0.05 & $<0.01$ \\
Single- $t$ modelling uncertainties & 0.18 & 0.02 & $<0.01$ \\
Other modelling uncertainties & 0.05 & 0.01 & $<0.01$ \\
Jets & 0.20 & 0.06 & 0.01 \\
-tagging & 0.01 & 0.01 & 0.04 \\
$E_{\mathrm{T}}^{\text {miss }}$ soft term and pile-up & $<0.01$ & $<0.01$ & $<0.01$ \\
Other experimental systematic uncertainties & 0.01 & $<0.01$ & $<0.01$ \\
Signal systematic uncertainties & $<0.01$ & $<0.01$ & $<0.01$ \\
MC sample size & 0.08 & 0.07 & 0.11 \\
\hline Statistical uncertainty & 0.27 & 0.61 & 0.79 \\
Total systematic uncertainties & 0.73 & 0.39 & 0.21 \\
\hline
\end{tabular}

Table 3. Relative importance of the different sources of uncertainty for different $Z^{\prime}$-2HDMs, with the masses of the $Z^{\prime}$ boson and the $A$ boson given in the second row, expressed as fractional impact on the signal strength parameter. The fractional impact is calculated by considering the square of the uncertainty in the signal strength parameter arising from a given group of uncertainties (as listed in the left column of the table), divided by the square of the total uncertainty in the signal strength parameter. Due to correlations, the sum of the different impacts of systematic uncertainties might not add up to the total impact of all systematic uncertainties.

The distributions of the Higgs boson candidate mass $m_{h}$ after the background-only fit are shown in figures 4 and 5 . The signal to background ratio is higher in the $\geq 3 b$-tag signal regions, because the $2 \mathrm{HDM}+a$ signal model is shown with $\tan \beta=10$, where $b$-associated production dominates. Tables 4 and 5 present the background estimates in comparison with the observed data. No significant deviation from SM expectations is observed, with the largest deficit corresponding to a local significance of $2.3 \sigma$, and the largest excess amounting to $1.6 \sigma$. Figure 6 summarises the total yields in the signal regions as a function of $E_{\mathrm{T}}^{\mathrm{miss}}$. The background prediction from simulation is scaled upwards in the fit for lower $E_{\mathrm{T}}^{\text {miss }}$ values, while the simulation agrees better with the data for large $E_{\mathrm{T}}^{\text {miss }}$ values.

The results are interpreted as exclusion limits at 95\% CL in the $Z^{\prime}-2 \mathrm{HDM}$ and the $2 \mathrm{HDM}+a$ scenarios in figures 7 and 8 . Considering the $Z^{\prime}$-2HDM case, $Z^{\prime}$ masses up to 3 $\mathrm{TeV}$ are excluded for $A$ masses of $300 \mathrm{GeV}$ at $95 \% \mathrm{CL}$. The exclusion boundaries for the $2 \mathrm{HDM}+a$ scenario extend up to $m_{a}=520 \mathrm{GeV}$ for $m_{A}=1.25 \mathrm{TeV}$ for $g g \mathrm{~F}$ production and $\tan \beta=1$. This is an improvement of about $200 \mathrm{GeV}$ in $m_{a}$ on previous results [123], which reinterpreted the earlier $h(\rightarrow b \bar{b})+E_{\mathrm{T}}^{\text {miss }}$ analysis using $36.1 \mathrm{fb}^{-1}[27]$. 


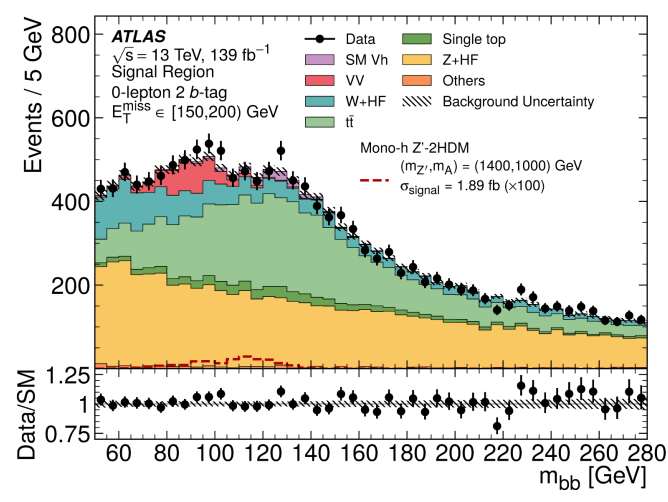

(a)

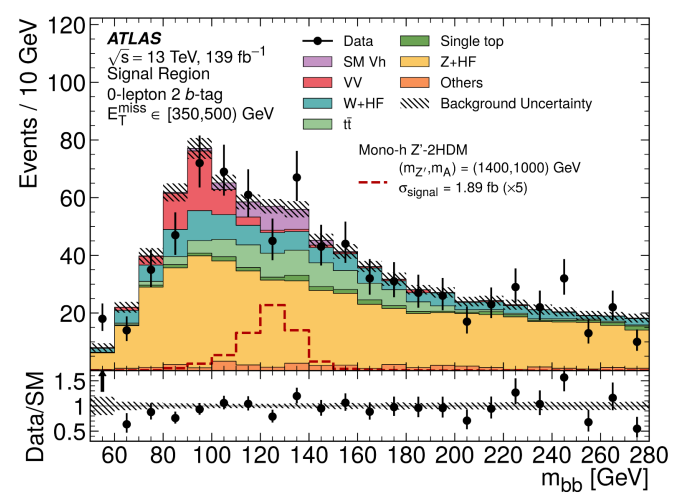

(c)

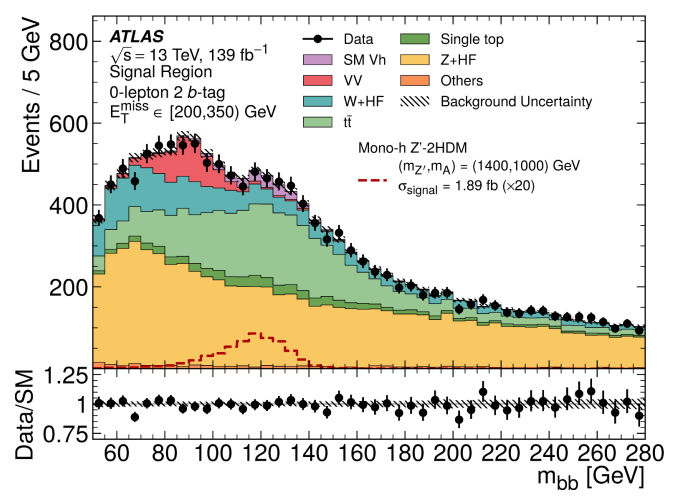

(b)

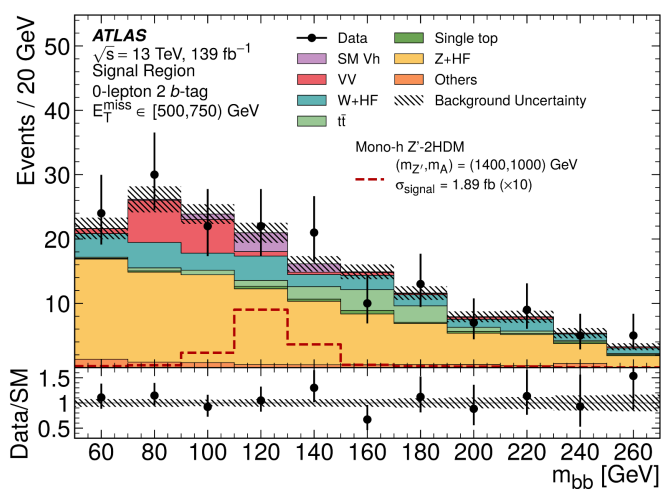

(d)

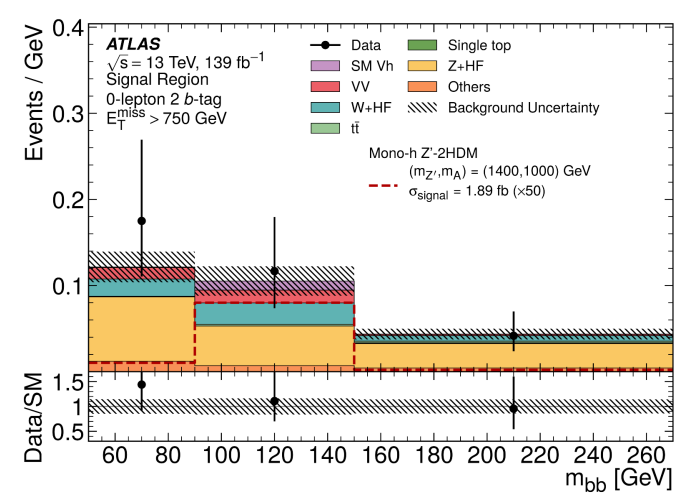

(e)

Figure 4. Distributions of the Higgs boson candidate mass in the $2 b$-tag signal regions for different $E_{\mathrm{T}}^{\text {miss }}$ ranges. The top panel comparies the fitted background yields with data, while the bottom panel indicates the ratio of the observed data to the predicted Standard Model backgrounds. The background yields are obtained in a background-only fit to data. An example signal model from the $Z^{\prime}-2 \mathrm{HDM}$ with parameters $\left(m_{Z^{\prime}}, m_{A}\right)=(1400 \mathrm{GeV}, 1000 \mathrm{GeV})$ and a cross-section of $1.89 \mathrm{fb}$ is displayed for comparison. The signal is scaled by the factors indicated in the legend for better visibility. 


\begin{tabular}{|l|c|c|c|c|c|}
\hline $2 b$-tag signal regions & {$[150,200) \mathrm{GeV}$} & {$[200,350) \mathrm{GeV}$} & {$[350,500) \mathrm{GeV}$} & {$[500,750) \mathrm{GeV}$} & $>750 \mathrm{GeV}$ \\
$E_{\mathrm{T}}^{\text {miss }}$ range & $6470 \pm 310$ & $7200 \pm 310$ & $507 \pm 26$ & $94 \pm 7$ & $9.2 \pm 1.8$ \\
$Z+\mathrm{HF}$ & $72 \pm 15$ & $137 \pm 29$ & $18 \pm 4$ & $4.5 \pm 1.0$ & $1.17 \pm 0.30$ \\
$Z+$ light jets & $1590 \pm 210$ & $1760 \pm 230$ & $106 \pm 14$ & $25 \pm 4$ & $3.1 \pm 0.6$ \\
$W+\mathrm{HF}$ & $86 \pm 35$ & $92 \pm 35$ & $14 \pm 5$ & $1.6 \pm 0.6$ & $0.21 \pm 0.09$ \\
$W+$ light jets & $570 \pm 260$ & $570 \pm 260$ & $21 \pm 10$ & $2.6 \pm 1.9$ & $0.10 \pm 0.16$ \\
Single top-quark & $4680 \pm 290$ & $3280 \pm 240$ & $76 \pm 9$ & $11.4 \pm 1.6$ & $0.38 \pm 0.08$ \\
$t \bar{t}$ & $450 \pm 50$ & $600 \pm 60$ & $56 \pm 7$ & $15.2 \pm 1.9$ & $1.61 \pm 0.29$ \\
Diboson & $151 \pm 10$ & $202 \pm 12$ & $26.6 \pm 1.8$ & $5.6 \pm 0.5$ & $0.68 \pm 0.12$ \\
$V h$ & $7.6 \pm 0.4$ & $11.8 \pm 0.5$ & $0.45 \pm 0.06$ & $0.286 \pm 0.029$ & $0.035 \pm 0.006$ \\
$t \bar{t}+V / h$ & $14070 \pm 110$ & $13860 \pm 100$ & $825 \pm 19$ & $160 \pm 8$ & $16.7 \pm 1.9$ \\
\hline Total background & 14259 & 13724 & 799 & 168 & 19 \\
\hline Data & & &
\end{tabular}

Table 4. Background yields in comparison with data in the $2 b$-tag signal regions for different $E_{\mathrm{T}}^{\text {miss }}$ ranges after a background-only fit to data. Statistical and systematic uncertainties are reported together.

\begin{tabular}{|l|c|c|c|c|}
\hline$\geq 3$-tag signal regions & {$[150,200) \mathrm{GeV}$} & {$[200,350) \mathrm{GeV}$} & {$[350,500) \mathrm{GeV}$} & $>500 \mathrm{GeV}$ \\
$E_{\mathrm{T}}^{\text {miss }}$ range & $102 \pm 15$ & $278 \pm 28$ & $26.4 \pm 3.5$ & $15.6 \pm 1.9$ \\
$Z+\mathrm{HF}$ & $0.6 \pm 0.4$ & $2.9 \pm 0.8$ & $0.34 \pm 0.12$ & $0.46 \pm 0.12$ \\
$Z+$ light jets & $21 \pm 4$ & $47 \pm 9$ & $4.2 \pm 0.9$ & $2.4 \pm 0.4$ \\
$W+\mathrm{HF}$ & $0.01 \pm 0.04$ & $1.7 \pm 0.9$ & $0.8 \pm 0.4$ & $0.031 \pm 0.026$ \\
$W+$ light jets & $276 \pm 19$ & $252 \pm 22$ & $5.1 \pm 0.7$ & $17.9 \pm 1.8$ \\
$t \bar{t}$ & $23 \pm 11$ & $55 \pm 25$ & $2.9 \pm 1.4$ & $3.4 \pm 1.7$ \\
Single top-quark & $4.8 \pm 1.4$ & $12.9 \pm 2.2$ & $1.8 \pm 0.4$ & $1.26 \pm 0.31$ \\
Diboson & $0.65 \pm 0.28$ & $2.9 \pm 0.5$ & $0.40 \pm 0.08$ & $0.230 \pm 0.025$ \\
$V h$ & $1.78 \pm 0.17$ & $3.89 \pm 0.26$ & $0.371 \pm 0.035$ & $0.78 \pm 0.08$ \\
$t \bar{t}+V / h$ & $430 \pm 15$ & $656 \pm 21$ & $42 \pm 4$ & $42.0 \pm 2.8$ \\
\hline Total background & 408 & 658 & 42 & 46 \\
\hline Data & \multicolumn{3}{|c}{} \\
\hline
\end{tabular}

Table 5. Background yields in comparison with data in the $\geq 3 b$-tag signal regions for different $E_{\mathrm{T}}^{\text {miss }}$ ranges after a background-only fit to data. Statistical and systematic uncertainties are reported together.

The higher exclusion limit at high $m_{A}$, low $m_{a}$, is due to an increase of the cross-section of the $a \rightarrow a h$ process, without resonant $A$ production. It should be noted that with the exact parameter choices adopted in this analysis, the aah coupling becomes larger than $4 \pi$ for $m_{A} \gtrsim 1750 \mathrm{GeV}$. Moreover, as discussed in refs. [25, 26] values of $m_{A} \gtrsim 1250 \mathrm{GeV}$ (for $\tan \beta=1$ ) or $m_{A} \gtrsim 2150 \mathrm{GeV}$ (for $\tan \beta=10$ ) would not be consistent with the requirement of having a bounded-from-below scalar potential, given the parameter choices discussed in this paper. These constraints can be relaxed substantially if the quartic couplings assume a value closer to the perturbativity limit and also in more general $2 \mathrm{HDMs}$ containing additional couplings as discussed in refs. [124, 125]. Therefore, the above should not be considered as a strong requirement for the validity of the model predictions. At 


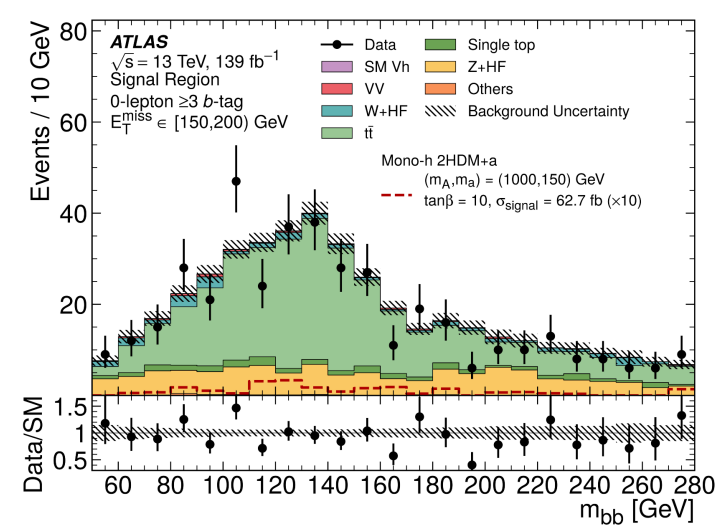

(a)

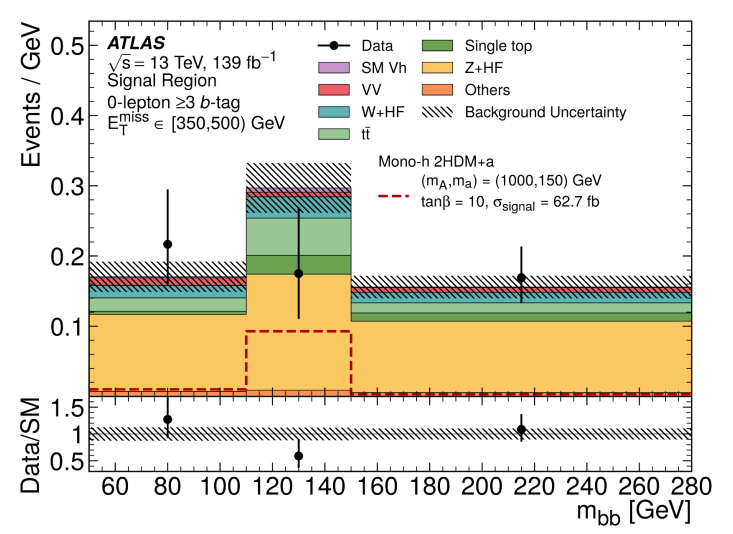

(c)

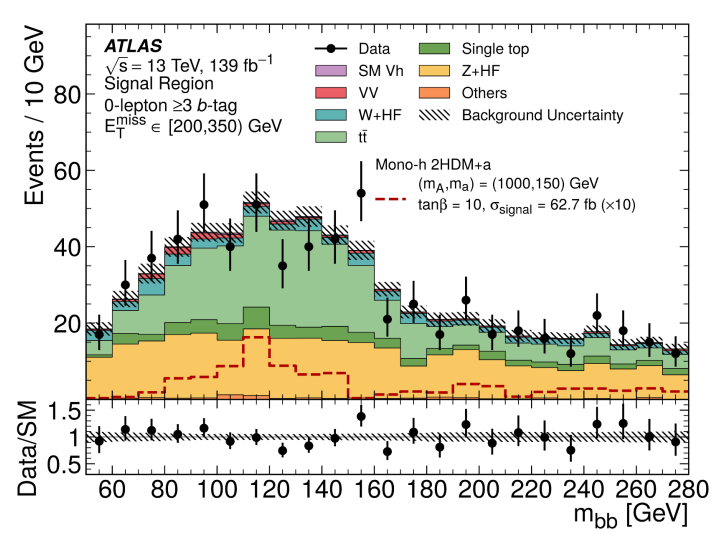

(b)

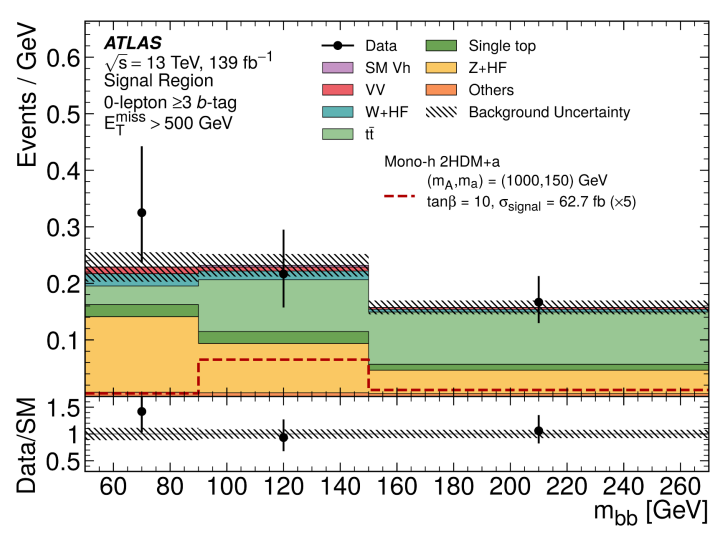

(d)

Figure 5. Distributions of the Higgs boson candidate mass in the $\geq 3 b$-tag signal regions. The top panel compares the fitted background yields with data, while the bottom panel indicates the ratio of the observed data to the predicted Standard Model backgrounds. The background yields are obtained in a background-only fit to data. An example signal model from the $2 \mathrm{HDM}+a$ with $b b A$ production and with parameters $\left(m_{A}, m_{a}\right)=(1000 \mathrm{GeV}, 150 \mathrm{GeV}), \tan \beta=10$, and a cross-section of $62.7 \mathrm{fb}$ is displayed for comparison. The signal is scaled by the factors indicated in the legend for better visibility.

high $m_{A}$ the width of the additional Higgs bosons grows substantially and the theoretical predictions are subject to additional theoretical uncertainties associated with the treatment of the width. Exclusion limits are therefore not shown in the region of very large widths $\left(m_{A}>2200 \mathrm{GeV}\right)$.

In the case of $b b A$ production and $\tan \beta=10$, the exclusion limits extend up to $m_{a}=240 \mathrm{GeV}$ for $m_{A}=900 \mathrm{GeV}$. The inclusion of the $\geq 3 b$-tag tag region helps to increase the sensitivity relative to the $2 b$-tag tag region by about $30-70 \%$. The difference between observed and expected limits arises from data deficits in the $\geq 3 b$-tag region, especially the deficit around the Higgs boson peak in the $E_{\mathrm{T}}^{\text {miss }} \in[350,500) \mathrm{GeV}$ region, as shown in figure 5 . The $2 \mathrm{HDM}+a$ scenario with $b b A$ production and $\tan \beta=10$ is considered for the signatures discussed in this paper for the first time. 


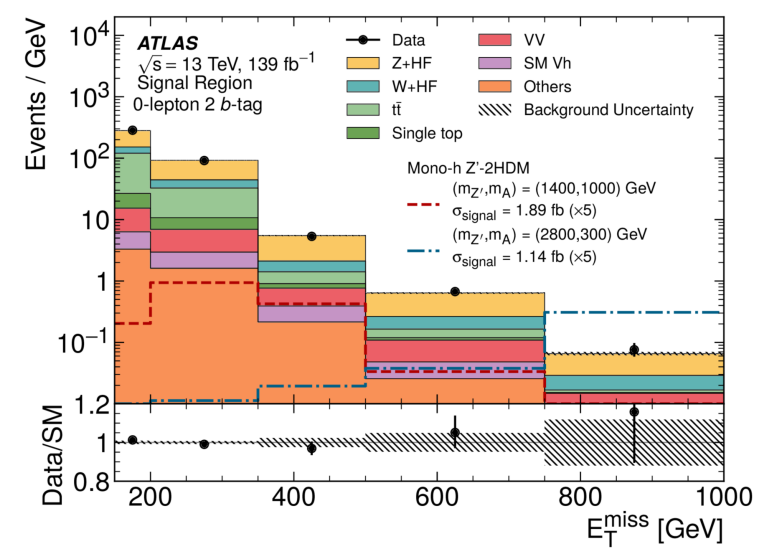

(a)

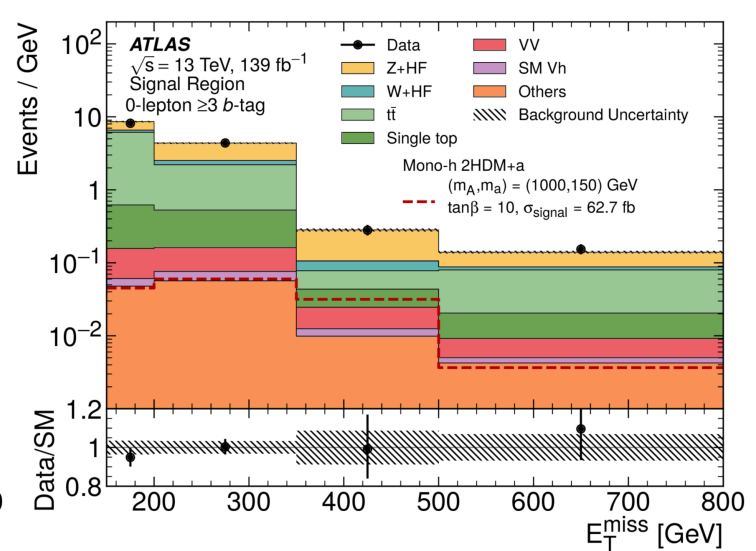

(b)

Figure 6. $E_{\mathrm{T}}^{\text {miss }}$ distributions after requiring (a) two $b$-tagged jets or (b) at least three $b$-tagged jets. The background yields are obtained in a background-only fit to data. Each bin corresponds to one signal region, where the bins at lower $E_{\mathrm{T}}^{\text {miss }}$ use small- $R$ jets and the ones with larger $E_{\mathrm{T}}^{\text {miss }}$ select events with at least one large- $R$ jet. The rightmost bin includes all events with $E_{\mathrm{T}}^{\text {miss }}$ above the range shown on the plots. The bottom panel compares the data with background estimates as obtained from the fit. Example signal models are overlaid in the top panel. In plot (a) two $Z^{\prime}$-2HDM points are shown, one with parameters $\left(m_{Z^{\prime}}, m_{A}\right)=(1400 \mathrm{GeV}, 1000 \mathrm{GeV})$ and a cross-section of $1.89 \mathrm{fb}$ and the other with parameters $\left(m_{Z^{\prime}}, m_{A}\right)=(2800 \mathrm{GeV}, 300 \mathrm{GeV})$ and a cross-section of $1.14 \mathrm{fb}$. Both signals are scaled up by a factor of 5 for better visibility. In plot (b) a $2 \mathrm{HDM}+a$ is shown with parameters $\left(m_{A}, m_{a}\right)=(1000 \mathrm{GeV}, 150 \mathrm{GeV}), \tan \beta=10$ and a cross-section of $62.7 \mathrm{fb}$.

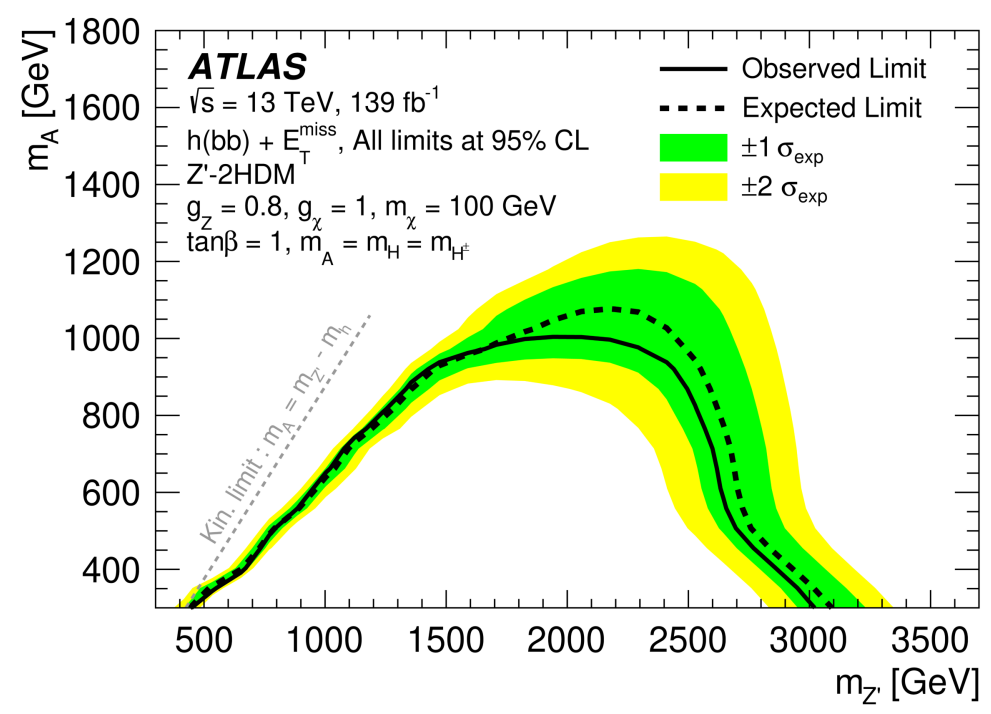

Figure 7. Exclusion limits in the $Z^{\prime}-2 \mathrm{HDM}$ model. The solid black line shows the observed limit at $95 \% \mathrm{CL}$, the dashed black line the expected limit. The green band gives the $\pm 1 \sigma$ uncertainties of the expected limit, the yellow band the $\pm 2 \sigma$ uncertainties. 


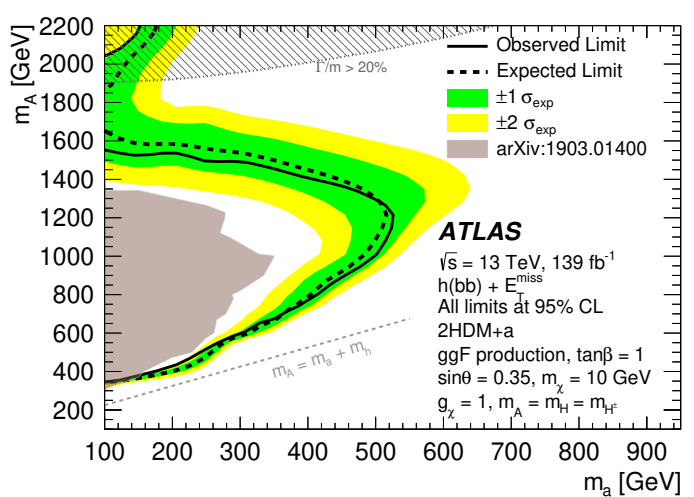

(a)

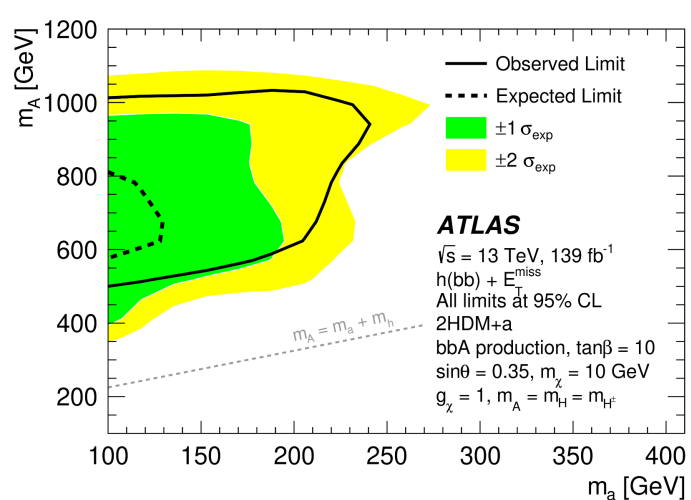

(b)

Figure 8. Exclusion limits for the $2 \mathrm{HDM}+a$ signal with (a) $\tan \beta=1$ and $g g \mathrm{~F}$ production and with (b) $\tan \beta=10$ and $b b A$ production. The solid black line shows the observed limit at $95 \%$ $\mathrm{CL}$, the dashed black line the expected limit. The green band gives the $\pm 1 \sigma$ uncertainties of the expected limit, the yellow band the $\pm 2 \sigma$ uncertainties. The hashed area in plot (a) indicates the region where the width of at least one of the Higgs bosons $A, H$ or $H^{ \pm}$or of the pseudoscalar $a$ is above $20 \%$ of its mass. Extending the exclusion into this region would require additional assumptions within the $2 \mathrm{HDM}+a$ model, and as such limits are not shown beyond $2.2 \mathrm{TeV}$.

Figure 9 displays the upper limits on the visible cross-section, defined as

$$
\sigma_{\mathrm{vis}, h(b \bar{b})+\mathrm{DM}} \equiv \sigma_{h+\mathrm{DM}} \times \mathscr{B}(h \rightarrow b \bar{b}) \times(\mathscr{A} \times \varepsilon)
$$

where $(\mathscr{A} \times \varepsilon)$ with the acceptance $\mathscr{A}$ and the reconstruction efficiency $\varepsilon$ quantifies the probability for a certain event to be reconstructed within a window around the Higgs boson mass in a given signal region. The visible cross-section is obtained from the number of signal events in each signal region extracted from a fit to the $m(b \bar{b})$ distribution as described below, divided by the integrated luminosity.

In contrast to the model-specific exclusion limits in figures 7 and 8 , the upper limits on the visible cross-section are calculated without dependence on a signal model. They only assume that a resonance was produced with a mass close to $125 \mathrm{GeV}$ and decays into a pair of $b$ quarks in association with $E_{\mathrm{T}}^{\text {miss }}$. For this purpose, the binning in $m_{h}$ in the signal regions is modified such that all bins under the Higgs boson peak in a range from $90 \mathrm{GeV}$ to $150 \mathrm{GeV}$ are merged into one bin. This bin includes the Higgs boson peak, but excludes important parts of the $Z$ boson peak. A simultaneous fit to all control and signal regions is performed, using the modified binning. However, in order not to assume a specific signal model, a signal may only be present in the one bin under the Higgs boson peak. Although all signal regions are fitted simultaneously, the signal contributions in the different signal regions are independent of each other, which is ensured by using different signal strength parameters.

\section{Conclusion}

A search for dark matter production in association with a Higgs boson decaying into $b \bar{b}$ is presented, considering final states with either two or at least three $b$-tagged jets and without 


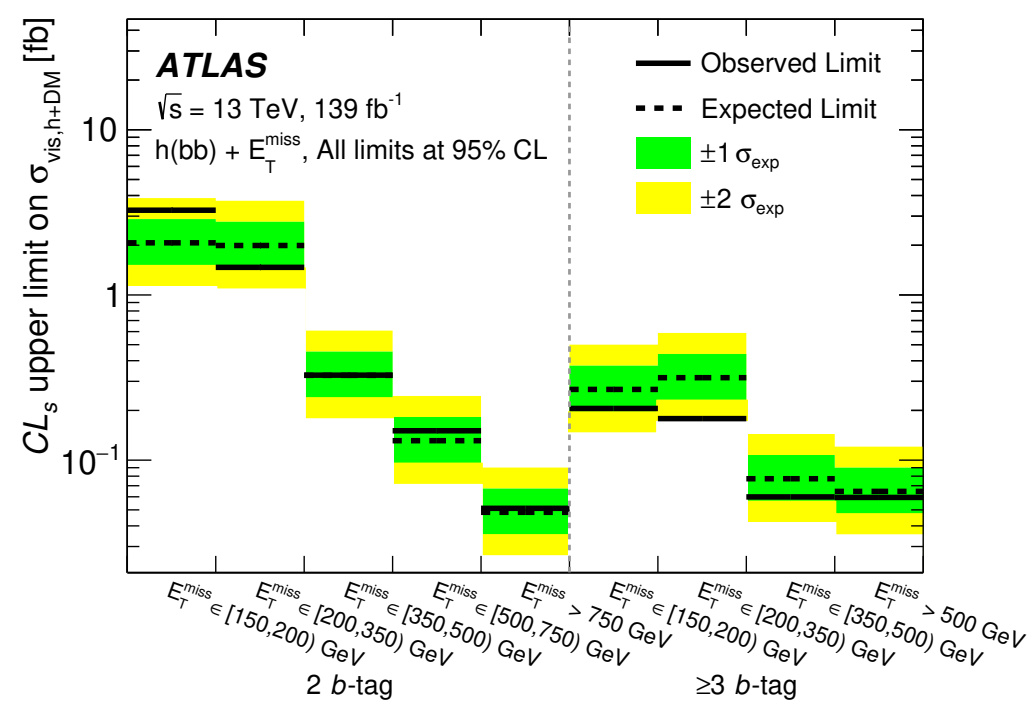

Figure 9. Model-independent upper limits on the visible cross-section $\sigma_{\mathrm{vis}, h(b \bar{b})+\mathrm{DM}} \equiv \sigma_{h+\mathrm{DM}} \times$ $\mathscr{B}(h \rightarrow b \bar{b}) \times \mathscr{A} \times \varepsilon$ in the different signal regions.

leptons. The search uses proton-proton collision data recorded by the ATLAS experiment at the LHC during the data-taking periods in 2015-2018, corresponding to an integrated luminosity of $139 \mathrm{fb}^{-1}$. The analysis targets the $2 \mathrm{HDM}$ extended by dark matter particles and either a heavy vector boson $Z^{\prime}\left(Z^{\prime}-2 \mathrm{HDM}\right)$ or a pseudoscalar singlet $a(2 \mathrm{HDM}+a)$ as mediator particle.

Sensitivity to these models is obtained by considering different $b$-jet multiplicity regions, and the cases of boosted or non-boosted Higgs bosons. Further improvements relative to previous searches targeting this signature with a partial dataset from Run 2 of the LHC are obtained through better background rejection of $t \bar{t}$ processes, better identification of $b$-hadrons from the decay of a boosted Higgs boson, and improved suppression of multijet processes by using an object-based $E_{\mathrm{T}}^{\text {miss }}$ significance.

No significant deviation from Standard Model expectations was found. Exclusion limits are set on the $Z^{\prime}-2 \mathrm{HDM}$ and extend up to $Z^{\prime}$-masses of $3 \mathrm{TeV}$ at $95 \% \mathrm{CL}$. This is an improvement of about $700 \mathrm{GeV}$ relative to the analysis in ref. [27] for heavy $Z^{\prime}$ mediator masses. In the case of the $2 \mathrm{DHM}+a$, the mass of the pseudoscalar $a$ is excluded up to $520 \mathrm{GeV}$ for $\tan \beta=1$ and gluon-gluon fusion production, and up to $300 \mathrm{GeV}$ for $\tan \beta=10$ in $b$-associated production. Upper limits on the visible cross-section range from 0.05 to $3.26 \mathrm{fb}$, depending on the signal region.

\section{Acknowledgments}

We thank CERN for the very successful operation of the LHC, as well as the support staff from our institutions without whom ATLAS could not be operated efficiently.

We acknowledge the support of ANPCyT, Argentina; YerPhI, Armenia; ARC, Australia; BMWFW and FWF, Austria; ANAS, Azerbaijan; SSTC, Belarus; CNPq and 
FAPESP, Brazil; NSERC, NRC and CFI, Canada; CERN; ANID, Chile; CAS, MOST and NSFC, China; COLCIENCIAS, Colombia; MSMT CR, MPO CR and VSC CR, Czech Republic; DNRF and DNSRC, Denmark; IN2P3-CNRS and CEA-DRF/IRFU, France; SRNSFG, Georgia; BMBF, HGF and MPG, Germany; GSRT, Greece; RGC and Hong Kong SAR, China; ISF and Benoziyo Center, Israel; INFN, Italy; MEXT and JSPS, Japan; CNRST, Morocco; NWO, Netherlands; RCN, Norway; MNiSW and NCN, Poland; FCT, Portugal; MNE/IFA, Romania; JINR; MES of Russia and NRC KI, Russian Federation; MESTD, Serbia; MSSR, Slovakia; ARRS and MIZŠ, Slovenia; DST/NRF, South Africa; MICINN, Spain; SRC and Wallenberg Foundation, Sweden; SERI, SNSF and Cantons of Bern and Geneva, Switzerland; MOST, Taiwan; TAEK, Turkey; STFC, United Kingdom; DOE and NSF, United States of America. In addition, individual groups and members have received support from BCKDF, CANARIE, Compute Canada, CRC and IVADO, Canada; Beijing Municipal Science \& Technology Commission, China; COST, ERC, ERDF, Horizon 2020 and Marie Skłodowska-Curie Actions, European Union; Investissements d'Avenir Labex, Investissements d'Avenir Idex and ANR, France; DFG and AvH Foundation, Germany; Herakleitos, Thales and Aristeia programmes co-financed by EU-ESF and the Greek NSRF, Greece; BSF-NSF and GIF, Israel; La Caixa Banking Foundation, CERCA Programme Generalitat de Catalunya and PROMETEO and GenT Programmes Generalitat Valenciana, Spain; Göran Gustafssons Stiftelse, Sweden; The Royal Society and Leverhulme Trust, United Kingdom.

The crucial computing support from all WLCG partners is acknowledged gratefully, in particular from CERN, the ATLAS Tier-1 facilities at TRIUMF (Canada), NDGF (Denmark, Norway, Sweden), CC-IN2P3 (France), KIT/GridKA (Germany), INFN-CNAF (Italy), NL-T1 (Netherlands), PIC (Spain), ASGC (Taiwan), RAL (U.K.) and BNL (U.S.A.), the Tier-2 facilities worldwide and large non-WLCG resource providers. Major contributors of computing resources are listed in ref. [126].

Open Access. This article is distributed under the terms of the Creative Commons Attribution License (CC-BY 4.0), which permits any use, distribution and reproduction in any medium, provided the original author(s) and source are credited.

\section{References}

[1] D. Clowe, A. Gonzalez and M. Markevitch, Weak lensing mass reconstruction of the interacting cluster 1E0657-558: direct evidence for the existence of dark matter, Astrophys. J. 604 (2004) 596 [astro-ph/0312273] [INSPIRE].

[2] Planck collaboration, Planck 2015 results. XIII. Cosmological parameters, Astron. Astrophys. 594 (2016) A13 [arXiv:1502.01589] [inSPIRE].

[3] XENON collaboration, Dark matter search results from a one ton-year exposure of XENON1T, Phys. Rev. Lett. 121 (2018) 111302 [arXiv:1805.12562] [INSPIRE].

[4] PandaX-II collaboration, Dark matter results from first 98.7 days of data from the PandaX-II experiment, Phys. Rev. Lett. 117 (2016) 121303 [arXiv: 1607.07400] [INSPIRE].

[5] DARKSIDE collaboration, Low-mass dark matter search with the DarkSide-50 experiment, Phys. Rev. Lett. 121 (2018) 081307 [arXiv:1802. 06994] [INSPIRE]. 
[6] XENON collaboration, Search for light dark matter interactions enhanced by the Migdal effect or Bremsstrahlung in XENON1T, Phys. Rev. Lett. 123 (2019) 241803 [arXiv: 1907.12771] [INSPIRE].

[7] PICO collaboration, Dark matter search results from the complete exposure of the PICO-60 $C_{3} F_{8}$ bubble chamber, Phys. Rev. D 100 (2019) 022001 [arXiv:1902.04031] [INSPIRE].

[8] FERMI-LAT collaboration, Searching for dark matter annihilation from Milky Way dwarf spheroidal galaxies with six years of Fermi Large Area Telescope data, Phys. Rev. Lett. 115 (2015) 231301 [arXiv: 1503.02641] [INSPIRE].

[9] L. Evans and P. Bryant, eds., LHC machine, 2008 JINST 3 S08001 [INSPIRE].

[10] ATLAS collaboration, Search for new phenomena in events with an energetic jet and missing transverse momentum in pp collisions at $\sqrt{s}=13$ TeV with the ATLAS detector, Phys. Rev. D 103 (2021) 112006 [arXiv:2102.10874] [InSPIRE].

[11] CMS collaboration, Search for new physics in final states with an energetic jet or a hadronically decaying $W$ or $Z$ boson and transverse momentum imbalance at $\sqrt{s}=13 \mathrm{TeV}$, Phys. Rev. D 97 (2018) 092005 [arXiv:1712.02345] [INSPIRE].

[12] CDF collaboration, A Search for dark matter in events with one jet and missing transverse energy in p $\bar{p}$ collisions at $\sqrt{s}=1.96$ TeV, Phys. Rev. Lett. 108 (2012) 211804 [arXiv:1203.0742] [INSPIRE].

[13] ATLAS collaboration, Search for dark matter in events with a hadronically decaying vector boson and missing transverse momentum in pp collisions at $\sqrt{s}=13$ TeV with the ATLAS detector, JHEP 10 (2018) 180 [arXiv:1807.11471] [INSPIRE].

[14] CMS collaboration, Search for dark matter produced with an energetic jet or a hadronically decaying $W$ or $Z$ boson at $\sqrt{s}=13 \mathrm{TeV}$, JHEP 07 (2017) 014 [arXiv:1703.01651] [INSPIRE].

[15] ATLAS collaboration, Search for an invisibly decaying Higgs boson or dark matter candidates produced in association with a $Z$ boson in pp collisions at $\sqrt{s}=13$ TeV with the ATLAS detector, Phys. Lett. B 776 (2018) 318 [arXiv: 1708.09624] [INSPIRE].

[16] ATLAS collaboration, Search for dark matter in association with an energetic photon in pp collisions at $\sqrt{s}=13 \mathrm{TeV}$ with the ATLAS detector, JHEP 02 (2021) 226 [arXiv:2011.05259] [INSPIRE].

[17] CMS collaboration, Search for new physics in the monophoton final state in proton-proton collisions at $\sqrt{s}=13 \mathrm{TeV}$, JHEP 10 (2017) 073 [arXiv:1706.03794] [InSPIRE].

[18] CDF collaboration, Search for large extra dimensions in final states containing one photon or jet and large missing transverse energy produced in p $\bar{p}$ collisions at $\sqrt{s}=1.96$ TeV, Phys. Rev. Lett. 101 (2008) 181602 [arXiv:0807.3132] [INSPIRE].

[19] L. Carpenter, A. DiFranzo, M. Mulhearn, C. Shimmin, S. Tulin and D. Whiteson, Mono-Higgs-boson: a new collider probe of dark matter, Phys. Rev. D 89 (2014) 075017 [arXiv:1312.2592] [INSPIRE].

[20] G. Bertone, D. Hooper and J. Silk, Particle dark matter: evidence, candidates and constraints, Phys. Rept. 405 (2005) 279 [hep-ph/0404175] [INSPIRE].

[21] C.P. Burgess, M. Pospelov and T. ter Veldhuis, The minimal model of nonbaryonic dark matter: A Singlet scalar, Nucl. Phys. B 619 (2001) 709 [hep-ph/0011335] [INSPIRE].

[22] J. March-Russell, S.M. West, D. Cumberbatch and D. Hooper, Heavy dark matter through the Higgs portal, JHEP 07 (2008) 058 [arXiv:0801.3440] [INSPIRE]. 
[23] G.C. Branco, P.M. Ferreira, L. Lavoura, M.N. Rebelo, M. Sher and J.P. Silva, Theory and phenomenology of two-Higgs-doublet models, Phys. Rept. 516 (2012) 1 [arXiv:1106.0034] [INSPIRE].

[24] A. Berlin, T. Lin and L.-T. Wang, Mono-Higgs detection of dark matter at the LHC, JHEP 06 (2014) 078 [arXiv: 1402.7074] [INSPIRE].

[25] LHC DARK MatTer Working Group collaboration, LHC dark matter working group: next-generation spin-0 dark matter models, Phys. Dark Univ. 27 (2020) 100351 [arXiv: 1810.09420] [INSPIRE].

[26] M. Bauer, U. Haisch and F. Kahlhoefer, Simplified dark matter models with two Higgs doublets: I. Pseudoscalar mediators, JHEP 05 (2017) 138 [arXiv:1701.07427] [INSPIRE].

[27] ATLAS collaboration, Search for dark matter produced in association with a Higgs boson decaying to b $\bar{b}$ using $36 \mathrm{fb}^{-1}$ of pp collisions at $\sqrt{s}=13 \mathrm{TeV}$ with the ATLAS Detector, Phys. Rev. Lett. 119 (2017) 181804 [arXiv:1707.01302] [INSPIRE].

[28] CMS collaboration, Search for dark matter particles produced in association with a Higgs boson in proton-proton collisions at $\sqrt{s}=13 \mathrm{TeV}$, JHEP 03 (2020) 025 [arXiv: 1908.01713] [INSPIRE].

[29] ATLAS collaboration, Search for dark matter in events with missing transverse momentum and a Higgs boson decaying into two photons in pp collisions at $\sqrt{s}=13 \mathrm{TeV}$ with the ATLAS detector, JHEP 10 (2021) 013 [arXiv:2104.13240] [INSPIRE].

[30] CMS collaboration, Search for dark matter produced in association with a Higgs boson decaying to $\gamma \gamma$ or $\tau^{+} \tau^{-}$at $\sqrt{s}=13 \mathrm{TeV}$, JHEP 09 (2018) 046 [arXiv:1806.04771] [INSPIRE].

[31] ATLAS collaboration, Jet reconstruction and performance using particle flow with the ATLAS detector, Eur. Phys. J. C 77 (2017) 466 [arXiv:1703.10485] [InSPIRE].

[32] ATLAS collaboration, Optimisation and performance studies of the ATLAS b-tagging algorithms for the 2017-18 LHC run, ATL-PHYS-PUB-2017-013 (2017).

[33] ATLAS collaboration, Identification of hadronic tau lepton decays using neural networks in the ATLAS experiment, Tech. Rep. ATL-PHYS-PUB-2019-033, CERN, Geneva (Aug, 2019).

[34] D. Krohn, J. Thaler and L.-T. Wang, Jets with variable R, JHEP 06 (2009) 059 [arXiv:0903.0392] [INSPIRE].

[35] ATLAS collaboration, Object-based missing transverse momentum significance in the ATLAS detector, ATLAS-CONF-2018-038 (2018).

[36] ATLAS collaboration, The ATLAS experiment at the CERN Large Hadron Collider, 2008 JINST 3 S08003 [INSPIRE].

[37] ATLAS collaboration, ATLAS Insertable B-Layer technical design report, ATLAS-TDR-2010-19 (2010).

[38] ATLAS IBL collaboration, Production and integration of the ATLAS Insertable B-Layer, 2018 JINST 13 T05008 [arXiv: 1803.00844] [INSPIRE].

[39] ATLAS collaboration, Performance of the ATLAS Trigger System in 2015, Eur. Phys. J. $C 77$ (2017) 317 [arXiv:1611.09661] [INSPIRE].

[40] ATLAS collaboration, The ATLAS collaboration software and firmware, ATL-SOFT-PUB-2021-001 (2021).

[41] ATLAS collaboration, Luminosity determination in pp collisions at $\sqrt{s}=13 \mathrm{TeV}$ using the ATLAS detector at the LHC, ATLAS-CONF-2019-021 (2019). 
[42] G. Avoni et al., The new LUCID-2 detector for luminosity measurement and monitoring in ATLAS, 2018 JINST 13 P07017 [INSPIRE].

[43] ATLAS collaboration, ATLAS data quality operations and performance for 2015-2018 data-taking, 2020 JINST 15 P04003 [arXiv: 1911.04632] [INSPIRE].

[44] ATLAS collaboration, Performance of the missing transverse momentum triggers for the ATLAS detector during Run-2 data taking, JHEP 08 (2020) 080 [arXiv:2005.09554] [INSPIRE].

[45] J. Alwall et al., The automated computation of tree-level and next-to-leading order differential cross sections, and their matching to parton shower simulations, JHEP $\mathbf{0 7}$ (2014) 079 [arXiv: 1405.0301] [inSPIRE].

[46] T. Sjöstrand et al., An introduction to PYTHIA 8.2, Comput. Phys. Commun. 191 (2015) 159 [arXiv: 1410.3012] [INSPIRE].

[47] ATLAS collaboration, ATLAS PYTHIA 8 tunes to 7 TeV data, ATL-PHYS-PUB-2014-021 (2014).

[48] D. Abercrombie et al., Dark Matter benchmark models for early LHC Run-2 searches: report of the ATLAS/CMS dark matter forum, Phys. Dark Univ. 27 (2020) 100371 [arXiv:1507.00966] [INSPIRE].

[49] ATLAS and CMS collaborations, Combined measurement of the Higgs boson mass in pp collisions at $\sqrt{s}=7$ and $8 \mathrm{TeV}$ with the ATLAS and CMS experiments, Phys. Rev. Lett. 114 (2015) 191803 [arXiv: 1503.07589] [INSPIRE].

[50] SHERPA collaboration, Event Generation with Sherpa 2.2, SciPost Phys. 7 (2019) 034 [arXiv: 1905.09127] [INSPIRE].

[51] T. Gleisberg and S. Hoeche, Comix, a new matrix element generator, JHEP 12 (2008) 039 [arXiv:0808.3674] [INSPIRE].

[52] F. Cascioli, P. Maierhofer and S. Pozzorini, Scattering amplitudes with open loops, Phys. Rev. Lett. 108 (2012) 111601 [arXiv:1111.5206] [INSPIRE].

[53] A. Denner, S. Dittmaier and L. Hofer, Collier: a Fortran-based Complex One-Loop LIbrary in Extended Regularizations, Comput. Phys. Commun. 212 (2017) 220 [arXiv:1604.06792] [INSPIRE].

[54] S. Schumann and F. Krauss, A parton shower algorithm based on Catani-Seymour dipole factorisation, JHEP 03 (2008) 038 [arXiv:0709.1027] [INSPIRE].

[55] S. Hoeche, F. Krauss, M. Schonherr and F. Siegert, A critical appraisal of $N L O+P S$ matching methods, JHEP 09 (2012) 049 [arXiv:1111.1220] [INSPIRE].

[56] S. Hoeche, F. Krauss, M. Schonherr and F. Siegert, QCD matrix elements + parton showers: the NLO case, JHEP 04 (2013) 027 [arXiv: 1207.5030] [INSPIRE].

[57] S. Catani, F. Krauss, R. Kuhn and B.R. Webber, QCD matrix elements + parton showers, JHEP 11 (2001) 063 [hep-ph/0109231] [INSPIRE].

[58] S. Hoeche, F. Krauss, S. Schumann and F. Siegert, QCD matrix elements and truncated showers, JHEP 05 (2009) 053 [arXiv:0903.1219] [InSPIRE].

[59] S. Frixione, P. Nason and G. Ridolfi, A positive-weight next-to-leading-order Monte Carlo for heavy flavour hadroproduction, JHEP 09 (2007) 126 [arXiv: 0707.3088] [INSPIRE].

[60] P. Nason, A new method for combining NLO QCD with shower Monte Carlo algorithms, JHEP 11 (2004) 040 [hep-ph/0409146] [INSPIRE]. 
[61] S. Frixione, P. Nason and C. Oleari, Matching NLO QCD computations with Parton Shower simulations: the POWHEG method, JHEP 11 (2007) 070 [arXiv: 0709. 2092] [INSPIRE].

[62] S. Alioli, P. Nason, C. Oleari and E. Re, A general framework for implementing NLO calculations in shower Monte Carlo programs: the POWHEG BOX, JHEP 06 (2010) 043 [arXiv: 1002.2581] [INSPIRE].

[63] E. Re, Single-top Wt-channel production matched with parton showers using the POWHEG method, Eur. Phys. J. C 71 (2011) 1547 [arXiv:1009.2450] [inSPIRE].

[64] R. Frederix, E. Re and P. Torrielli, Single-top t-channel hadroproduction in the four-flavour scheme with POWHEG and aMC@NLO, JHEP 09 (2012) 130 [arXiv:1207.5391] [INSPIRE].

[65] S. Alioli, P. Nason, C. Oleari and E. Re, NLO single-top production matched with shower in POWHEG: s- and t-channel contributions, JHEP 09 (2009) 111 [Erratum ibid. 02 (2010) 011] [arXiv: 0907.4076] [INSPIRE].

[66] H.B. Hartanto, B. Jager, L. Reina and D. Wackeroth, Higgs boson production in association with top quarks in the POWHEG BOX, Phys. Rev. D 91 (2015) 094003 [arXiv: 1501.04498] [INSPIRE].

[67] M. Beneke, P. Falgari, S. Klein and C. Schwinn, Hadronic top-quark pair production with NNLL threshold resummation, Nucl. Phys. B 855 (2012) 695 [arXiv:1109.1536] [INSPIRE].

[68] M. Cacciari, M. Czakon, M. Mangano, A. Mitov and P. Nason, Top-pair production at hadron colliders with next-to-next-to-leading logarithmic soft-gluon resummation, Phys. Lett. B 710 (2012) 612 [arXiv:1111.5869] [INSPIRE].

[69] P. Bärnreuther, M. Czakon and A. Mitov, Percent level precision physics at the Tevatron: first genuine NNLO QCD corrections to $q \bar{q} \rightarrow t \bar{t}+X$, Phys. Rev. Lett. 109 (2012) 132001 [arXiv: 1204.5201] [INSPIRE].

[70] M. Czakon and A. Mitov, NNLO corrections to top-pair production at hadron colliders: the all-fermionic scattering channels, JHEP 12 (2012) 054 [arXiv:1207.0236] [INSPIRE].

[71] M. Czakon and A. Mitov, NNLO corrections to top pair production at hadron colliders: the quark-gluon reaction, JHEP 01 (2013) 080 [arXiv:1210.6832] [INSPIRE].

[72] M. Czakon, P. Fiedler and A. Mitov, Total top-quark pair-production cross section at hadron colliders through $O\left(\alpha_{S}^{4}\right)$, Phys. Rev. Lett. 110 (2013) 252004 [arXiv:1303.6254] [INSPIRE].

[73] M. Czakon and A. Mitov, Top++: a program for the calculation of the top-pair cross-section at hadron colliders, Comput. Phys. Commun. 185 (2014) 2930 [arXiv:1112.5675] [INSPIRE].

[74] M. Aliev, H. Lacker, U. Langenfeld, S. Moch, P. Uwer and M. Wiedermann, HATHOR: HAdronic Top and Heavy quarks crOss section calculatoR, Comput. Phys. Commun. 182 (2011) 1034 [arXiv: 1007.1327] [inSPIRE].

[75] P. Kant et al., HatHor for single top-quark production: Updated predictions and uncertainty estimates for single top-quark production in hadronic collisions, Comput. Phys. Commun. 191 (2015) 74 [arXiv: 1406.4403] [INSPIRE].

[76] LHC Higgs Cross Section Working Group collaboration, Handbook of LHC Higgs cross sections: 4. Deciphering the nature of the Higgs sector, arXiv:1610.07922 [INSPIRE].

[77] S. Frixione, E. Laenen, P. Motylinski, B.R. Webber and C.D. White, Single-top hadroproduction in association with a W boson, JHEP 07 (2008) 029 [arXiv:0805.3067] [INSPIRE]. 
[78] K. Hamilton, P. Nason and G. Zanderighi, MINLO: Multi-Scale Improved NLO, JHEP 10 (2012) 155 [arXiv: 1206.3572] [INSPIRE].

[79] G. Luisoni, P. Nason, C. Oleari and F. Tramontano, $H W^{ \pm} / H Z+0$ and 1 jet at $N L O$ with the POWHEG BOX interfaced to GoSam and their merging within MiNLO, JHEP 10 (2013) 083 [arXiv: 1306.2542] [InSPIRE].

[80] L. Altenkamp, S. Dittmaier, R.V. Harlander, H. Rzehak and T.J.E. Zirke, Gluon-induced Higgs-strahlung at next-to-leading order QCD, JHEP 02 (2013) 078 [arXiv:1211.5015] [INSPIRE].

[81] R.V. Harlander, A. Kulesza, V. Theeuwes and T. Zirke, Soft gluon resummation for gluon-induced Higgs Strahlung, JHEP 11 (2014) 082 [arXiv:1410.0217] [INSPIRE].

[82] O. Brein, R.V. Harlander and T.J.E. Zirke, vh@nnlo-Higgs Strahlung at hadron colliders, Comput. Phys. Commun. 184 (2013) 998 [arXiv:1210.5347] [INSPIRE].

[83] NNPDF collaboration, Parton distributions for the LHC Run II, JHEP 04 (2015) 040 [arXiv: 1410.8849] [INSPIRE].

[84] ATLAS collaboration, Studies on top-quark Monte Carlo modelling for Top2016, ATL-PHYS-PUB-2016-020 (2016).

[85] J. Pumplin, D.R. Stump, J. Huston, H.L. Lai, P.M. Nadolsky and W.K. Tung, New generation of parton distributions with uncertainties from global QCD analysis, JHEP 07 (2002) 012 [hep-ph/0201195] [inSPIRE].

[86] ATLAS collaboration, Measurement of the $Z / \gamma^{*}$ boson transverse momentum distribution in pp collisions at $\sqrt{s}=7 \mathrm{TeV}$ with the ATLAS detector, JHEP 09 (2014) 145 [arXiv: 1406.3660] [INSPIRE].

[87] D.J. Lange, The EvtGen particle decay simulation package, Nucl. Instrum. Meth. A 462 (2001) 152 [INSPIRE].

[88] ATLAS collaboration, The PYTHIA 8 A3 tune description of ATLAS minimum bias and inelastic measurements incorporating the Donnachie-Landshoff diffractive model, Tech. Rep. ATL-PHYS-PUB-2016-017, CERN, Geneva (Aug, 2016).

[89] ATLAS collaboration, The ATLAS simulation infrastructure, Eur. Phys. J. C 70 (2010) 823 [arXiv: 1005.4568] [INSPIRE].

[90] GEANT4 collaboration, GEANT4 - A simulation toolkit, Nucl. Instrum. Meth. A 506 (2003) 250 [INSPIRE].

[91] ATLAS collaboration, Vertex reconstruction performance of the ATLAS detector at $\sqrt{s}=13 \mathrm{TeV}$, ATL-PHYS-PUB-2015-026 (2015).

[92] M. Cacciari, G.P. Salam and G. Soyez, The anti-k $k_{t}$ jet clustering algorithm, JHEP 04 (2008) 063 [arXiv: 0802.1189] [INSPIRE].

[93] M. Cacciari, G.P. Salam and G. Soyez, FastJet user manual, Eur. Phys. J. C 72 (2012) 1896 [arXiv: 1111.6097] [INSPIRE].

[94] ATLAS collaboration, Performance of pile-up mitigation techniques for jets in pp collisions at $\sqrt{s}=8 \mathrm{TeV}$ using the ATLAS detector, Eur. Phys. J. C 76 (2016) 581 [arXiv: 1510.03823] [INSPIRE].

[95] ATLAS collaboration, Topological cell clustering in the ATLAS calorimeters and its performance in LHC Run 1, Eur. Phys. J. C 77 (2017) 490 [arXiv: 1603. 02934] [INSPIRE].

[96] D. Krohn, J. Thaler and L.-T. Wang, Jet trimming, JHEP 02 (2010) 084 [arXiv:0912.1342] [INSPIRE]. 
[97] M. Cacciari, G.P. Salam and G. Soyez, The catchment area of jets, JHEP 04 (2008) 005 [arXiv: 0802.1188] [INSPIRE].

[98] ATLAS collaboration, Jet energy scale and resolution measured in proton-proton collisions at $\sqrt{s}=13 \mathrm{TeV}$ with the ATLAS detector, Eur. Phys. J. C 81 (2021) 689 [arXiv: 2007.02645] [INSPIRE].

[99] ATLAS collaboration, Evidence for the $H \rightarrow b \bar{b}$ decay with the ATLAS detector, JHEP 12 (2017) 024 [arXiv: 1708.03299] [INSPIRE].

[100] ATLAS collaboration, Electron and photon performance measurements with the ATLAS detector using the 2015-2017 LHC proton-proton collision data, 2019 JINST 14 P12006 [arXiv: 1908.00005] [INSPIRE].

[101] ATLAS collaboration, Electron reconstruction and identification in the ATLAS experiment using the 2015 and 2016 LHC proton-proton collision data at $\sqrt{s}=13 \mathrm{TeV}$, Eur. Phys. J. C 79 (2019) 639 [arXiv:1902.04655] [INSPIRE].

[102] ATLAS collaboration, Muon reconstruction and identification efficiency in ATLAS using the full Run 2 pp collision data set at $\sqrt{s}=13$ TeV, Eur. Phys. J. C 81 (2021) 578 [arXiv: 2012.00578] [INSPIRE].

[103] ATLAS collaboration, Reconstruction, energy calibration, and identification of hadronically decaying tau leptons in the ATLAS experiment for Run-2 of the LHC, ATL-PHYS-PUB-2015-045 (2015).

[104] ATLAS collaboration, $E_{T}^{\text {miss }}$ performance in the ATLAS detector using 2015-2016 LHC p-p collisions, ATLAS-CONF-2018-023 (2018).

[105] ATLAS collaboration, Selection of jets produced in $13 \mathrm{TeV}$ proton-proton collisions with the ATLAS detector, ATLAS-CONF-2015-029 (2015).

[106] ATLAS collaboration, Performance of the ATLAS muon triggers in Run 2, 2020 JINST 15 P09015 [arXiv: 2004.13447] [INSPIRE].

[107] ATLAS collaboration, Performance of electron and photon triggers in ATLAS during LHC Run 2, Eur. Phys. J. C 80 (2020) 47 [arXiv: 1909.00761] [INSPIRE].

[108] W. Verkerke and D. Kirkby, The RooFit toolkit for data modeling, (2003).

[109] L. Moneta et al., The RooStats Project, PoS (ACAT2010) 057 [arXiv:1009.1003] [INSPIRE].

[110] G. Cowan, K. Cranmer, E. Gross and O. Vitells, Asymptotic formulae for likelihood-based tests of new physics, Eur. Phys. J. C 71 (2011) 1554 [Erratum ibid. 73 (2013) 2501] [arXiv: 1007.1727] [INSPIRE].

[111] A.L. Read, Presentation of search results: the $C L_{s}$ technique, J. Phys. G 28 (2002) 2693 [INSPIRE].

[112] ATLAS collaboration, In situ calibration of large-radius jet energy and mass in $13 \mathrm{TeV}$ proton-proton collisions with the ATLAS detector, Eur. Phys. J. C 79 (2019) 135 [arXiv: 1807. 09477] [INSPIRE].

[113] ATLAS collaboration, In-situ measurements of the ATLAS large-radius jet response in 13 $\mathrm{TeV}$ pp collisions, ATLAS-CONF-2017-063 (2017).

[114] ATLAS collaboration, ATLAS b-jet identification performance and efficiency measurement with $t \bar{t}$ events in pp collisions at $\sqrt{s}=13 \mathrm{TeV}$, Eur. Phys. J. C 79 (2019) 970 [arXiv: 1907.05120$]$ [INSPIRE].

[115] ATLAS collaboration, Measurement of b-tagging efficiency of c-jets in t $\bar{t}$ events using a likelihood approach with the ATLAS detector, ATLAS-CONF-2018-001 (2018). 
[116] ATLAS collaboration, Calibration of light-flavour b-jet mistagging rates using ATLAS proton-proton collision data at $\sqrt{s}=13 \mathrm{TeV}$, ATLAS-CONF-2018-006 (2018).

[117] ATLAS collaboration, Performance of missing transverse momentum reconstruction with the ATLAS detector using proton-proton collisions at $\sqrt{s}=13$ TeV, Eur. Phys. J. C 78 (2018) 903 [arXiv:1802.08168] [INSPIRE].

[118] M. Bahr et al., HERWIG++ physics and manual, Eur. Phys. J. C 58 (2008) 639 [arXiv: 0803.0883] [INSPIRE].

[119] J. Bellm et al., HERWIG \%.0/HERWIG++ 3.0 release note, Eur. Phys. J. C 76 (2016) 196 [arXiv: 1512.01178] [INSPIRE].

[120] T. Sjöstrand, S. Mrenna and P.Z. Skands, A brief introduction to PYTHIA 8.1, Comput. Phys. Commun. 178 (2008) 852 [arXiv:0710.3820] [INSPIRE].

[121] J. Butterworth et al., PDF4LHC recommendations for LHC Run II, J. Phys. G 43 (2016) 023001 [arXiv: 1510.03865] [INSPIRE].

[122] ATLAS collaboration, Measurements of the production cross-section for a $Z$ boson in association with b-jets in proton-proton collisions at $\sqrt{s}=13 \mathrm{TeV}$ with the ATLAS detector, JHEP 07 (2020) 044 [arXiv: 2003.11960] [INSPIRE].

[123] ATLAS collaboration, Constraints on mediator-based dark matter and scalar dark energy models using $\sqrt{s}=13 \mathrm{TeV}$ pp collision data collected by the ATLAS detector, JHEP 05 (2019) 142 [arXiv: 1903.01400] [INSPIRE].

[124] M. Bauer, M. Klassen and V. Tenorth, Universal properties of pseudoscalar mediators in dark matter extensions of 2HDMs, JHEP 07 (2018) 107 [arXiv: 1712.06597] [INSPIRE].

[125] U. Haisch and G. Polesello, Searching for heavy Higgs bosons in the $t \bar{t} Z$ and $t b W$ final states, JHEP 09 (2018) 151 [arXiv: 1807.07734] [INSPIRE].

[126] ATLAS collaboration, ATLAS computing acknowledgements, ATL-SOFT-PUB-2020-001 (2020). 


\section{The ATLAS collaboration}

G. $\operatorname{Aad}^{99}$, B. Abbott ${ }^{126}$, D.C. Abbott ${ }^{100}$, A. Abed Abud ${ }^{34}$, K. Abeling ${ }^{51}$, D.K. Abhayasinghe ${ }^{91}$, S.H. Abidi ${ }^{27}$, O.S. AbouZeid ${ }^{38}$, H. Abramowicz ${ }^{159}$, H. Abreu ${ }^{158}$, Y. Abulaiti ${ }^{5}$, A.C. Abusleme Hoffman ${ }^{144 a}$, B.S. Acharya $64 a, 64 b, p$, B. Achkar ${ }^{51}$, L. Adam ${ }^{97}$, C. Adam Bourdarios ${ }^{4}$, L. Adamczyk ${ }^{81 a}$, L. Adamek ${ }^{164}$, J. Adelman ${ }^{118}$, A. Adiguzel ${ }^{11 c, a e}$, S. Adorni ${ }^{52}$, T. Adye ${ }^{141}$, A.A. Affolder ${ }^{143}$, Y. Afik ${ }^{158}$, C. Agapopoulou ${ }^{62}$, M.N. Agaras ${ }^{12}$, J. Agarwala 68a,68b, A. Aggarwal ${ }^{116}$, C. Agheorghiesei ${ }^{25 c}$, J.A. Aguilar-Saavedra 137f,137a,ad,

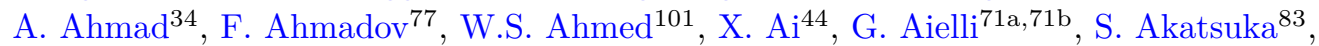
M. Akbiyik ${ }^{97}$, T.P.A. Åkesson ${ }^{94}$, A.V. Akimov ${ }^{108}$, K. Al Khoury ${ }^{37}$, G.L. Alberghi²1b, J. Albert ${ }^{173}$, M.J. Alconada Verzini ${ }^{86}$, S. Alderweireldt ${ }^{34}$, M. Aleksa ${ }^{34}$, I.N. Aleksandrov ${ }^{77}$, C. Alexa ${ }^{25 b}$, T. Alexopoulos ${ }^{9}$, A. Alfonsi ${ }^{117}$, F. Alfonsi ${ }^{21 b, 21 a}$, M. Alhroob ${ }^{126}$, B. Ali ${ }^{139}$, S. Ali $^{156}$, M. Aliev ${ }^{163}$, G. Alimonti66a, C. Allaire ${ }^{34}$, B.M.M. Allbrooke ${ }^{154}$, P.P. Allport ${ }^{19}$, A. Aloisio 67a,67b, F. Alonso ${ }^{86}$, C. Alpigiani ${ }^{146}$, E. Alunno Camelia ${ }^{71 a, 71 b}$, M. Alvarez Estevez ${ }^{96}$, M.G. Alviggi ${ }^{67 a, 67 b}$, Y. Amaral Coutinho ${ }^{78 b}$, A. Ambler ${ }^{101}$, L. Ambroz ${ }^{132}$, C. Amelung ${ }^{34}$, D. Amidei ${ }^{103}$, S.P. Amor Dos Santos ${ }^{137 a}$, S. Amoroso ${ }^{44}$, C.S. Amrouche ${ }^{52}$, C. Anastopoulos ${ }^{147}$, N. Andari ${ }^{142}$, T. Andeen ${ }^{10}$, J.K. Anders ${ }^{18}$, S.Y. Andrean ${ }^{43 a, 43 b}$, A. Andreazza ${ }^{66 a, 66 b}$, V. Andrei ${ }^{59 a}$, S. Angelidakis ${ }^{8}$, A. Angerami ${ }^{37}$, A.V. Anisenkov ${ }^{119 b, 119 a}$, A. Annovi ${ }^{69 a}$, C. Antel $^{52}$, M.T. Anthony ${ }^{147}$, E. Antipov ${ }^{127}$, M. Antonelli ${ }^{49}$, D.J.A. Antrim ${ }^{16}$, F. Anulli ${ }^{70 a}$, M. Aoki ${ }^{79}$, J.A. Aparisi Pozo ${ }^{171}$, M.A. Aparo ${ }^{154}$, L. Aperio Bella ${ }^{44}$, N. Aranzabal ${ }^{34}$, V. Araujo Ferraz ${ }^{78 a}$, C. Arcangeletti ${ }^{49}$, A.T.H. Arce ${ }^{47}$, E. Arena ${ }^{88}$, J-F. Arguin ${ }^{107}$, S. Argyropoulos ${ }^{50}$, J.-H. Arling ${ }^{44}$, A.J. Armbruster ${ }^{34}$, A. Armstrong ${ }^{168}$, O. Arnaez ${ }^{164}$, H. Arnold ${ }^{34}$, Z.P. Arrubarrena Tame ${ }^{111}$, G. Artoni ${ }^{132}$, H. Asada ${ }^{114}$, K. Asai ${ }^{124}$, S. Asai ${ }^{161}$, N.A. Asbah ${ }^{57}$, E.M. Asimakopoulou ${ }^{169}$, L. Asquith ${ }^{154}$, J. Assahsah ${ }^{33 d}$, K. Assamagan ${ }^{27}$, R. Astalos ${ }^{26 a}$, R.J. Atkin ${ }^{31 a}$, M. Atkinson ${ }^{170,}$ N.B. Atlay ${ }^{17}$, H. Atmani ${ }^{62}$, P.A. Atmasiddha ${ }^{103}$, K. Augsten ${ }^{139}$, S. Auricchio $67 a, 67 b$, V.A. Austrup ${ }^{179}$, G. Avolio ${ }^{34}$, M.K. Ayoub ${ }^{13 c}$, G. Azuelos ${ }^{107, a k}$, D. Babal ${ }^{26 a}$, H. Bachacou ${ }^{142}$, K. Bachas ${ }^{160}$, F. Backman ${ }^{43 a, 43 b}$, P. Bagnaia ${ }^{70 a, 70 b}$, H. Bahrasemani ${ }^{150}$, A.J. Bailey ${ }^{171}$, V.R. Bailey ${ }^{170}$, J.T. Baines ${ }^{141}$, C. Bakalis ${ }^{9}$, O.K. Baker ${ }^{180}$, P.J. Bakker ${ }^{117}$, E. Bakos ${ }^{14}$, D. Bakshi Gupta ${ }^{7}$, S. Balaji ${ }^{155}$, R. Balasubramanian ${ }^{117}$, E.M. Baldin 119b,119a , P. Balek ${ }^{140}$,

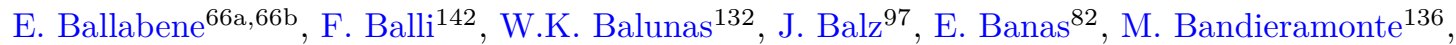
A. Bandyopadhyay ${ }^{17}$, L. Barak ${ }^{159}$, E.L. Barberio ${ }^{102}$, D. Barberis ${ }^{53 b, 53 a}$, M. Barbero ${ }^{99}$, G. Barbour ${ }^{92}$, K.N. Barends ${ }^{31 a}$, T. Barillari ${ }^{112}$, M-S. Barisits ${ }^{34}$, J. Barkeloo ${ }^{129}$, T. Barklow ${ }^{151}$, B.M. Barnett ${ }^{141}$, R.M. Barnett ${ }^{16}$, A. Baroncelli ${ }^{58 a}$, G. Barone ${ }^{27}$, A.J. Barr ${ }^{132}$,

L. Barranco Navarro ${ }^{43 a, 43 b}$, F. Barreiro ${ }^{96}$, J. Barreiro Guimarães da Costa ${ }^{13 a}$, U. Barron ${ }^{159}$, S. Barsov ${ }^{135}$, F. Bartels ${ }^{59 a}$, R. Bartoldus ${ }^{151}$, G. Bartolini ${ }^{99}$, A.E. Barton ${ }^{87}$, P. Bartos ${ }^{26 a}$, A. Basalaev ${ }^{44}$, A. Basan ${ }^{97}$, I. Bashta ${ }^{72 a, 72 b}$, A. Bassalat ${ }^{62}$, M.J. Basso ${ }^{164}$, C.R. Basson ${ }^{98}$, R.L. Bates ${ }^{55}$, S. Batlamous ${ }^{33 e}$, J.R. Batley ${ }^{30}$, B. Batool ${ }^{149}$, M. Battaglia ${ }^{143}$, M. Bauce ${ }^{70 a, 70 b}$, F. Bauer ${ }^{142, *}$, P. Bauer ${ }^{22}$, H.S. Bawa ${ }^{29}$, A. Bayirli ${ }^{11 c}$, J.B. Beacham ${ }^{47}$, T. Beau ${ }^{133}$, P.H. Beauchemin ${ }^{167}$, F. Becherer ${ }^{50}$, P. Bechtle ${ }^{22}$, H.P. Beck ${ }^{18, r}$, K. Becker ${ }^{175}$, C. Becot ${ }^{44}$, A.J. Beddall ${ }^{11 a}$, V.A. Bednyakov ${ }^{77}$, C.P. Bee ${ }^{153}$, T.A. Beermann ${ }^{179}$, M. Begalli ${ }^{78 b}$, M. Begel ${ }^{27}$, A. Behera ${ }^{153}$, J.K. Behr ${ }^{44}$, C. Beirao Da Cruz E Silva ${ }^{34}$, J.F. Beirer ${ }^{51,34}$, F. Beisiegel ${ }^{22}$, M. Belfkir ${ }^{4}$, G. Bella ${ }^{159}$, L. Bellagamba ${ }^{21 b}$, A. Bellerive ${ }^{32}$, P. Bellos ${ }^{19}$, K. Beloborodov ${ }^{119 b, 119 a}$, K. Belotskiy ${ }^{109}$, N.L. Belyaev ${ }^{109}$, D. Benchekroun ${ }^{33 a}$, Y. Benhammou ${ }^{159}$, D.P. Benjamin ${ }^{5}$, M. Benoit ${ }^{27}$, J.R. Bensinger ${ }^{24}$, S. Bentvelsen ${ }^{117}$, L. Beresford ${ }^{132}$, M. Beretta ${ }^{49}$, D. Berge ${ }^{17}$, E. Bergeaas Kuutmann ${ }^{169}$, N. Berger ${ }^{4}$, B. Bergmann ${ }^{139}$, L.J. Bergsten ${ }^{24}$, J. Beringer ${ }^{16}$, S. Berlendis ${ }^{6}$, G. Bernardi ${ }^{133}$, C. Bernius ${ }^{151}$, F.U. Bernlochner ${ }^{22}$, T. Berry ${ }^{91}$, P. Berta ${ }^{44}$, A. Berthold ${ }^{46}$, I.A. Bertram ${ }^{87}$, O. Bessidskaia Bylund ${ }^{179}$, S. Bethke ${ }^{112}$, A. Betti ${ }^{40}$, A.J. Bevan ${ }^{90}$, S. Bhatta ${ }^{153}$, D.S. Bhattacharya ${ }^{174}$, P. Bhattarai ${ }^{24}$, V.S. Bhopatkar ${ }^{5}$, R. Bi ${ }^{136}$, R.M. Bianchi ${ }^{136}$, 


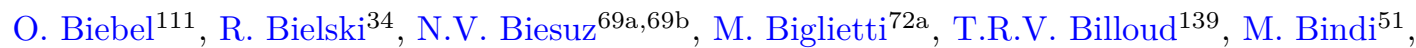
A. Bingul ${ }^{11 d}$, C. Bini ${ }^{70 a, 70 b}$, S. Biondi $i^{21 b, 21 a}$, C.J. Birch-sykes ${ }^{98}$, G.A. Bird ${ }^{19,141}$, M. Birman ${ }^{177}$,

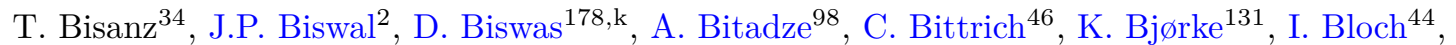
C. Blocker ${ }^{24}$, A. Blue ${ }^{55}$, U. Blumenschein ${ }^{90}$, J. Blumenthal ${ }^{97}$, G.J. Bobbink ${ }^{117}$, V.S. Bobrovnikov ${ }^{119 b, 119 a}$, D. Bogavac ${ }^{12}$, A.G. Bogdanchikov ${ }^{119 b, 119 a}$, C. Bohm ${ }^{43 a}$, V. Boisvert ${ }^{91}$, P. Bokan ${ }^{44}$, T. Bold ${ }^{81 a}$, M. Bomben ${ }^{133}$, M. Bona ${ }^{90}$, M. Boonekamp ${ }^{142}$, C.D. Booth ${ }^{91}$, A.G. Borbély ${ }^{55}$, H.M. Borecka-Bielska ${ }^{107}$, L.S. Borgna ${ }^{92}$, G. Borissov ${ }^{87}$, D. Bortoletto ${ }^{132}$, D. Boscherini ${ }^{21 b}$, M. Bosman ${ }^{12}$, J.D. Bossio Sola ${ }^{101}$, K. Bouaouda ${ }^{33 a}$, J. Boudreau ${ }^{136}$, E.V. Bouhova-Thacker ${ }^{87}$, D. Boumediene ${ }^{36}$, R. Bouquet ${ }^{133}$, A. Boveia ${ }^{125}$, J. Boyd ${ }^{34}$, D. Boye ${ }^{27}$, I.R. Boyko ${ }^{77}$, A.J. Bozson ${ }^{91}$, J. Bracinik ${ }^{19}$, N. Brahimi ${ }^{58 d, 58 c}$, G. Brandt ${ }^{179}$, O. Brandt ${ }^{30}$, F. Braren ${ }^{44}$, B. Brau ${ }^{100}$, J.E. Brau ${ }^{129}$, W.D. Breaden Madden ${ }^{55}$, K. Brendlinger ${ }^{44}$, R. Brener ${ }^{177}$, L. Brenner ${ }^{34}$, R. Brenner ${ }^{169}$, S. Bressler ${ }^{177}$, B. Brickwedde ${ }^{97}$, D.L. Briglin ${ }^{19}$, D. Britton ${ }^{55}$, D. Britzger ${ }^{112}$, I. Brock ${ }^{22}$, R. Brock ${ }^{104}$, G. Brooijmans ${ }^{37}$, W.K. Brooks ${ }^{144 f}$, E. Brost $^{27}$, P.A. Bruckman de Renstrom ${ }^{82}$, B. Brüers ${ }^{44}$, D. Bruncko ${ }^{26 \mathrm{~b}}$, A. Bruni ${ }^{21 b}$, G. Bruni ${ }^{21 b}$, M. Bruschi ${ }^{21 b}$, N. Bruscino ${ }^{70 a, 70 b}$, L. Bryngemark ${ }^{151}$, T. Buanes ${ }^{15}$, Q. Buat ${ }^{153}$, P. Buchholz ${ }^{149}$, A.G. Buckley ${ }^{55}$, I.A. Budagov ${ }^{77}$, M.K. Bugge ${ }^{131}$, O. Bulekov ${ }^{109}$, B.A. Bullard ${ }^{57}$, T.J. Burch ${ }^{118}$, S. Burdin ${ }^{88}$, C.D. Burgard ${ }^{44}$, A.M. Burger ${ }^{127}$, B. Burghgrave ${ }^{7}$, J.T.P. Burr ${ }^{44}$, C.D. Burton ${ }^{10}$, J.C. Burzynski ${ }^{100}$, V. Büscher ${ }^{97}$, P.J. Bussey ${ }^{55}$, J.M. Butler ${ }^{23}$, C.M. Buttar ${ }^{55}$, J.M. Butterworth ${ }^{92}$, W. Buttinger ${ }^{141}$, C.J. Buxo Vazquez ${ }^{104}$, A.R. Buzykaev ${ }^{119 b, 119 a}$, G. Cabras ${ }^{21 b}$, S. Cabrera Urbán ${ }^{171}$, D. Caforio ${ }^{54}$, H. Cai ${ }^{136}$, V.M.M. Cairo ${ }^{151}$, O. Cakir ${ }^{3 a}$, N. Calace $^{34}$, P. Calafiura ${ }^{16}$, G. Calderini ${ }^{133}$, P. Calfayan $^{63}$, G. Callea ${ }^{55}$, L.P. Caloba ${ }^{78 b}$, A. Caltabiano ${ }^{71 a, 71 b}$, S. Calvente Lopez $^{96}$, D. Calvet $^{36}$, S. Calvet ${ }^{36}$, T.P. Calvet ${ }^{99}$, M. Calvetti ${ }^{69 a, 69 b}$, R. Camacho Toro ${ }^{133}$, S. Camarda ${ }^{34}$, D. Camarero Munoz ${ }^{96}$, P. Camarri ${ }^{71 a, 71 b}$, M.T. Camerlingo ${ }^{72 a, 72 b}$, D. Cameron ${ }^{131}$, C. Camincher ${ }^{173}$, M. Campanelli ${ }^{92}$, A. Camplani ${ }^{38}$, V. Canale ${ }^{67 a, 67 b}$, A. Canesse ${ }^{101}$, M. Cano Bret ${ }^{75}$, J. Cantero ${ }^{127}$, Y. Cao ${ }^{170}$, M. Capua ${ }^{39 b, 39 a}$, R. Cardarelli ${ }^{71 a}$, F. Cardillo ${ }^{171}$, G. Carducci ${ }^{39 b, 39 a}$, T. Carli ${ }^{34}$, G. Carlino ${ }^{67 a}$, B.T. Carlson ${ }^{136}$, E.M. Carlson ${ }^{173,165 a}$, L. Carminati ${ }^{66 a, 66 b}$, M. Carnesale ${ }^{70 a, 70 b}$, R.M.D. Carney ${ }^{151}$, S. Caron ${ }^{116}$, E. Carquin ${ }^{144 f}$, S. Carrá ${ }^{44}$, G. Carratta ${ }^{21 b, 21 a}$, J.W.S. Carter ${ }^{164}$, T.M. Carter ${ }^{48}$, D. Casadei ${ }^{31 c}$, M.P. Casado ${ }^{12, h}$, A.F. Casha ${ }^{164}$, E.G. Castiglia ${ }^{180}$, F.L. Castillo ${ }^{171}$, L. Castillo Garcia ${ }^{12}$, V. Castillo Gimenez ${ }^{171}$, N.F. Castro ${ }^{137 a, 137 e}$, A. Catinaccio ${ }^{34}$, J.R. Catmore ${ }^{131}$, A. Cattai ${ }^{34}$, V. Cavaliere ${ }^{27}$, N. Cavalli ${ }^{21 b, 21 a}$, V. Cavasinni ${ }^{69 a, 69 b}$, E. Celebi ${ }^{11 b}$, F. Celli ${ }^{132}$, K. Cerny ${ }^{128}$, A.S. Cerqueira ${ }^{78 a}$, A. Cerri ${ }^{154}$, L. Cerrito ${ }^{71 a, 71 b}$, F. Cerutti ${ }^{16}$, A. Cervelli2 ${ }^{21 b}$, S.A. Cetin ${ }^{11 b}$, Z. Chadi ${ }^{33 a}$, D. Chakraborty ${ }^{118}$, M. Chala ${ }^{137 f}$, J. Chan ${ }^{178}$, W.S. Chan ${ }^{117}$, W.Y. Chan ${ }^{88}$, J.D. Chapman ${ }^{30}$, B. Chargeishvili ${ }^{157 b}$, D.G. Charlton ${ }^{19}$, T.P. Charman ${ }^{90}$, M. Chatterjee ${ }^{18}$, C.C. $\mathrm{Chau}^{32}$, S. $\mathrm{Chekanov}^{5}$, S.V. Chekulaev ${ }^{165 a}$, G.A. Chelkov ${ }^{77, a g}$, A. Chen ${ }^{103}$, B. Chen ${ }^{76}$, C. Chen ${ }^{58 a}$, C.H. Chen ${ }^{76}$, H. Chen ${ }^{13 c}$, H. Chen ${ }^{27}$, J. Chen ${ }^{58 a}$, J. Chen ${ }^{37}$, J. Chen ${ }^{24}$, S. Chen ${ }^{134}$, S.J. Chen ${ }^{13 c}$, X. Chen ${ }^{13 b}$, Y. Chen ${ }^{58 a}$, Y-H. Chen ${ }^{44}$, C.L. Cheng ${ }^{178}$, H.C. Cheng ${ }^{60 a}$, H.J. Cheng ${ }^{13 a}$, A. Cheplakov 77 , E. Cheremushkina ${ }^{44}$, R. Cherkaoui El Moursli ${ }^{33 e}$, E. Cheu ${ }^{6}$, K. Cheung ${ }^{61}$, L. Chevalier ${ }^{142}$, V. Chiarella ${ }^{49}$, G. Chiarelli69a , G. Chiodini ${ }^{65 a}$, A.S. Chisholm ${ }^{19}$, A. Chitan ${ }^{25 b}$, I. Chiu ${ }^{161}$, Y.H. Chiu ${ }^{173}$, M.V. Chizhov ${ }^{77, t}$, K. Choi ${ }^{10}$, A.R. Chomont ${ }^{70 a, 70 b}$, Y. $\mathrm{Chou}^{100}$, Y.S. Chow ${ }^{117}$, L.D. Christopher ${ }^{31 f}$, M.C. Chu ${ }^{60 a}$, X. Chu ${ }^{13 a, 13 d}$, J. Chudoba ${ }^{138}$, J.J. Chwastowski ${ }^{82}$, D. Cieri ${ }^{112}$, K.M. Ciesla ${ }^{82}$, V. Cindro ${ }^{89}$, I.A. Cioară ${ }^{25 b}$, A. Ciocio ${ }^{16}$, F. Cirotto ${ }^{67 a, 67 b}$, Z.H. Citron ${ }^{177,1}$, M. Citterio ${ }^{66 a}$, D.A. Ciubotaru ${ }^{25 b}$, B.M. Ciungu ${ }^{164}$, A. Clark ${ }^{52}$, P.J. Clark ${ }^{48}$, S.E. Clawson ${ }^{98}$, C. Clement ${ }^{43 a, 43 b}$, L. Clissa ${ }^{21 b, 21 a}$, Y. Coadou ${ }^{99}$, M. Cobal ${ }^{64 a, 64 c}$, A. Coccaro ${ }^{53 b}$, J. Cochran ${ }^{76}$, R.F. Coelho Barrue ${ }^{137 a}$, R. Coelho Lopes De Sa ${ }^{100}$, S. Coelli66a, H. Cohen ${ }^{159}$, A.E.C. Coimbra ${ }^{34}$, B. Cole $^{37}$, J. Collot $^{56}$, P. Conde Muiño ${ }^{137 a, 137 h}$, S.H. Connell ${ }^{31 c}$, I.A. Connelly ${ }^{55}$, E.I. Conroy ${ }^{132}$, F. Conventi ${ }^{67 a, a l}$, H.G. Cooke ${ }^{19}$, A.M. Cooper-Sarkar ${ }^{132}$, F. Cormier ${ }^{172}$, L.D. Corpe $^{92}$, M. Corradi ${ }^{70 a, 70 b}$, E.E. Corrigan ${ }^{94}$, F. Corriveau ${ }^{101, \text { aa }}$, 
M.J. Costa ${ }^{171}$, F. Costanza $^{4}$, D. Costanzo ${ }^{147}$, B.M. Cote ${ }^{125}$, G. Cowan ${ }^{91}$, J.W. Cowley ${ }^{30}$, J. Crane ${ }^{98}$, K. Cranmer ${ }^{123}$, R.A. Creager ${ }^{134}$, S. Crépé-Renaudin ${ }^{56}$, F. Crescioli ${ }^{133}$, M. Cristinziani ${ }^{149}$, M. Cristoforetti ${ }^{73 a, 73 b, b}$, V. Croft $^{167}$, G. Crosetti ${ }^{39 b, 39 a}$, A. Cueto $^{4}$, T. Cuhadar Donszelmann ${ }^{168}$, H. Cui ${ }^{13 a, 13 d}$, A.R. Cukierman ${ }^{151}$, W.R. Cunningham ${ }^{55}$, S. Czekierda ${ }^{82}$, P. Czodrowski ${ }^{34}$, M.M. Czurylo ${ }^{59 b}$, M.J. Da Cunha Sargedas De Sousa ${ }^{58 a}$, J.V. Da Fonseca Pinto ${ }^{78 b}$, C. Da Via ${ }^{98}$, W. Dabrowski ${ }^{81 a}$, T. Dado ${ }^{45}$, S. Dahbi ${ }^{31 f}$, T. Dai ${ }^{103}$, C. Dallapiccola ${ }^{100}$, M. Dam ${ }^{38}$, G. D'amen ${ }^{27}$, V. D'Amico ${ }^{72 a, 72 b}$, J. Damp ${ }^{97}$, J.R. Dandoy ${ }^{134}$, M.F. Daneri ${ }^{28}$, M. Danninger ${ }^{150}$, V. Dao ${ }^{34}$, G. Darbo ${ }^{53 b}$, S. Darmora ${ }^{5}$, A. Dattagupta ${ }^{129}$, S. D'Auria ${ }^{66 a, 66 b}$, C. David ${ }^{165 b}$, T. Davidek ${ }^{140}$, D.R. Davis ${ }^{47}$, B. Davis-Purcell ${ }^{32}$, I. Dawson ${ }^{90}$, K. De ${ }^{7}$, R. De Asmundis ${ }^{67 a}$, M. De Beurs ${ }^{117}$, S. De Castro ${ }^{21 b, 21 a}$, N. De Groot ${ }^{116}$, P. de Jong ${ }^{117}$, H. De la Torre ${ }^{104}$, A. De Maria ${ }^{13 c}$, D. De Pedis ${ }^{70 a}$, A. De Salvo ${ }^{70 a}$, U. De Sanctis ${ }^{71 a, 71 b}$, M. De Santis ${ }^{71 a, 71 b}$, A. De Santo ${ }^{154}$, J.B. De Vivie De Regie ${ }^{56}$, D.V. Dedovich ${ }^{77}$, J. Degens ${ }^{117}$, A.M. Deiana ${ }^{40}$, J. Del Peso ${ }^{96}$, Y. Delabat $\operatorname{Diaz}^{44}$, F. Deliot ${ }^{142}$, C.M. Delitzsch ${ }^{6}$, M. Della Pietra ${ }^{67 a, 67 b}$, D. Della Volpe ${ }^{52}$, A. Dell'Acqua ${ }^{34}$, L. Dell'Asta ${ }^{66 a, 66 b}$, M. Delmastro ${ }^{4}$, P.A. Delsart ${ }^{56}$, S. Demers ${ }^{180}$, M. Demichev ${ }^{77}$, S.P. Denisov ${ }^{120}$, L. D'Eramo ${ }^{118}$, D. Derendarz ${ }^{82}$,

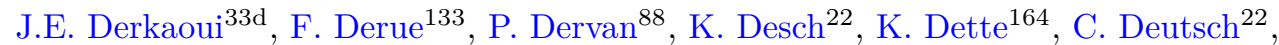
P.O. Deviveiros ${ }^{34}$, F.A. Di Bello ${ }^{70 a, 70 b}$, A. Di Ciaccio ${ }^{71 a, 71 b}$, L. Di Ciaccio ${ }^{4}$, C. Di Donato $67 a, 67 b$, A. Di Girolamo ${ }^{34}$, G. Di Gregorio ${ }^{69 a, 69 b}$, A. Di Luca ${ }^{73 a, 73 b}$, B. Di Micco ${ }^{72 a, 72 b}$, R. Di Nardo ${ }^{72 a, 72 b}$, C. Diaconu ${ }^{99}$, F.A. Dias ${ }^{117}$, T. Dias Do Vale ${ }^{137 a}$, M.A. Diaz ${ }^{144 a}$, F.G. Diaz Capriles $^{22}$, J. Dickinson ${ }^{16}$, M. Didenko ${ }^{171}$, E.B. Diehl ${ }^{103}$, J. Dietrich ${ }^{17}$, S. Díez Cornell ${ }^{44}$, C. Diez Pardos ${ }^{149}$, A. Dimitrievska ${ }^{16}$, W. Ding ${ }^{13 b}$, J. Dingfelder ${ }^{22}$, S.J. Dittmeier ${ }^{59 b}$, F. Dittus ${ }^{34}$, F. Djama ${ }^{99}$, T. Djobava ${ }^{157 b}$, J.I. Djuvsland ${ }^{15}$, M.A.B. Do Vale ${ }^{145}$, D. Dodsworth ${ }^{24}$, C. Doglioni ${ }^{94}$, J. Dolejsi ${ }^{140}$, Z. Dolezal ${ }^{140}$, M. Donadelli ${ }^{78 c}$, B. Dong ${ }^{58 c}$, J. Donini ${ }^{36}$, A. D'onofrio ${ }^{13 c}$, M. D'Onofrio ${ }^{88}$, J. Dopke ${ }^{141}$, A. Doria ${ }^{67 a}$, M.T. Dova ${ }^{86}$, A.T. Doyle ${ }^{55}$, E. Drechsler ${ }^{150}$,

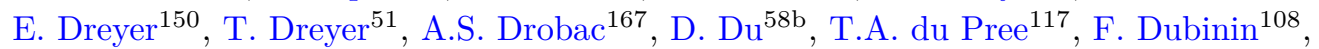
M. Dubovsky ${ }^{26 a}$, A. Dubreuil ${ }^{52}$, E. Duchovni ${ }^{177}$, G. Duckeck ${ }^{111}$, O.A. Ducu ${ }^{34,25 b}$, D. Duda ${ }^{112}$, A. Dudarev ${ }^{34}$, M. D’uffizi ${ }^{98}$, L. Duflot ${ }^{62}$, M. Dührssen ${ }^{34}$, C. Dülsen ${ }^{179}$, A.E. Dumitriu ${ }^{25 b}$, M. Dunford ${ }^{59 a}$, S. Dungs ${ }^{45}$, A. Duperrin ${ }^{99}$, H. Duran Yildiz ${ }^{3 a}$, M. Düren ${ }^{54}$, A. Durglishvili ${ }^{157 b}$, B. Dutta ${ }^{44}$, D. Duvnjak ${ }^{1}$, G.I. Dyckes ${ }^{134}$, M. Dyndal ${ }^{81 a}$, S. Dysch ${ }^{98}$, B.S. Dziedzic ${ }^{82}$, B. Eckerova ${ }^{26 a}$, M.G. Eggleston ${ }^{47}$, E. Egidio Purcino De Souza ${ }^{78 b}$, L.F. Ehrke ${ }^{52}$, T. Eifert ${ }^{7}$, G. Eigen ${ }^{15}$, K. Einsweiler ${ }^{16}$, T. Ekelof ${ }^{169}$, Y. El Ghazali ${ }^{33 b}$, H. El Jarrari ${ }^{33 e}$, A. El Moussaouy ${ }^{33 a}$, V. Ellajosyula ${ }^{169}$, M. Ellert ${ }^{169}$, F. Ellinghaus ${ }^{179}$, A.A. Elliot $^{90}$, N. Ellis $^{34}$, J. Elmsheuser ${ }^{27}$, M. Elsing ${ }^{34}$, D. Emeliyanov ${ }^{141}$, A. Emerman ${ }^{37}$, Y. Enari ${ }^{161}$, J. Erdmann ${ }^{45}$, A. Ereditato ${ }^{18}$, P.A. Erland ${ }^{82}$, M. Errenst ${ }^{179}$, M. Escalier ${ }^{62}$, C. Escobar ${ }^{171}$, O. Estrada Pastor ${ }^{171}$, E. Etzion ${ }^{159}$, G. Evans ${ }^{137 a}$, H. Evans ${ }^{63}$, M.O. Evans ${ }^{154}$, A. Ezhilov ${ }^{135}$, F. Fabbri ${ }^{55}$, L. Fabbri ${ }^{21 b, 21 a}$, V. Fabiani ${ }^{116}$, G. Facini ${ }^{175}$, R.M. Fakhrutdinov ${ }^{120}$, S. Falciano ${ }^{70 a}$, P.J. Falke ${ }^{22}$, S. Falke ${ }^{34}$, J. Faltova ${ }^{140}$, Y. Fan ${ }^{13 a}$, Y. Fang ${ }^{13 a}$, Y. Fang ${ }^{13 a}$, G. Fanourakis ${ }^{42}$, M. Fanti ${ }^{66 a, 66 b}$, M. Faraj ${ }^{58 c}$, A. Farbin ${ }^{7}$, A. Farilla ${ }^{72 a}$, E.M. Farina ${ }^{68 a, 68 b}$, T. Farooque ${ }^{104}$, S.M. Farrington ${ }^{48}$, P. Farthouat ${ }^{34}$, F. Fassi ${ }^{33 e}$, D. Fassouliotis ${ }^{8}$, M. Faucci Giannelli ${ }^{71 a, 71 b}$, W.J. Fawcett ${ }^{30}$, L. Fayard $^{62}$, O.L. Fedin ${ }^{135, \mathrm{q}}$, M. Feickert ${ }^{170}$, L. Feligioni ${ }^{99}$, A. Fell ${ }^{147}$, C. Feng ${ }^{58 b}$, M. Feng ${ }^{13 b}$, M.J. Fenton ${ }^{168}$, A.B. Fenyuk ${ }^{120}$, S.W. Ferguson ${ }^{41}$, J. Ferrando ${ }^{44}$, A. Ferrari ${ }^{169}$, P. Ferrari ${ }^{117}$, R. Ferrari6 ${ }^{68 a}$, D. Ferrere ${ }^{52}$, C. Ferretti ${ }^{103}$, F. Fiedler ${ }^{97}$, A. Filipčic ${ }^{89}$, F. Filthaut ${ }^{116}$, M.C.N. Fiolhais ${ }^{137 a, 137 c, a}$, L. Fiorini ${ }^{171}$, F. Fischer ${ }^{111}$, W.C. Fisher ${ }^{104}$, T. Fitschen ${ }^{19}$, I. Fleck ${ }^{149}$, P. Fleischmann ${ }^{103}$,

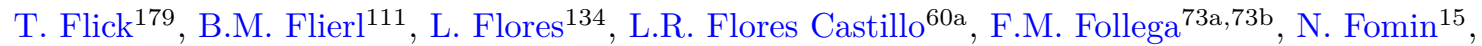
J.H. Foo ${ }^{164}$, G.T. Forcolin ${ }^{73 a, 73 b}$, B.C. Forland ${ }^{63}$, A. Formica ${ }^{142}$, F.A. Förster ${ }^{12}$, A.C. Forti ${ }^{98}$, E. Fortin ${ }^{99}$, M.G. Foti ${ }^{132}$, D. Fournier ${ }^{62}$, H. Fox ${ }^{87}$, P. Francavilla ${ }^{69 a, 69 b}$, S. Francescato ${ }^{70 a, 70 b}$, M. Franchini ${ }^{21 b, 21 a}$, S. Franchino ${ }^{59 a}$, D. Francis ${ }^{34}$, L. Franco ${ }^{4}$, L. Franconi ${ }^{18}$, M. Franklin ${ }^{57}$, G. Frattari ${ }^{70 a, 70 b}$, A.C. Freegard ${ }^{90}$, P.M. Freeman ${ }^{19}$, B. Freund ${ }^{107}$, W.S. Freund ${ }^{78 b}$, 
E.M. Freundlich ${ }^{45}$, D. Froidevaux ${ }^{34}$, J.A. Frost ${ }^{132}$, Y. Fu ${ }^{58 a}$, M. Fujimoto $^{124}$,

E. Fullana Torregrosa ${ }^{171}$, T. Fusayasu ${ }^{113}$, J. Fuster ${ }^{171}$, A. Gabrielli ${ }^{21 b, 21 a}$, A. Gabrielli ${ }^{34}$,

P. Gadow ${ }^{44}$, G. Gagliardi ${ }^{53 b, 53 a}$, L.G. Gagnon ${ }^{16}$, G.E. Gallardo ${ }^{132}$, E.J. Gallas ${ }^{132}$, B.J. Gallop ${ }^{141}$,

R. Gamboa Goni ${ }^{90}$, K.K. Gan ${ }^{125}$, S. Ganguly ${ }^{177}$, J. Gao ${ }^{58 a}$, Y. Gao ${ }^{48}$, Y.S. Gao ${ }^{29, n}$,

F.M. Garay Walls ${ }^{144 a}$, C. García ${ }^{171}$, J.E. García Navarro ${ }^{171}$, J.A. García Pascual ${ }^{13 a}$,

M. Garcia-Sciveres ${ }^{16}$, R.W. Gardner ${ }^{35}$, D. Garg ${ }^{75}$, S. Gargiulo ${ }^{50}$, C.A. Garner ${ }^{164}$, V. Garonne ${ }^{131}$, S.J. Gasiorowski ${ }^{146}$, P. Gaspar ${ }^{78 b}$, G. Gaudio ${ }^{68 a}$, P. Gauzzi ${ }^{70 a, 70 b}$, I.L. Gavrilenko ${ }^{108}$,

A. Gavrilyuk ${ }^{121}$, C. Gay ${ }^{172}$, G. Gaycken ${ }^{44}$, E.N. Gazis ${ }^{9}$, A.A. Geanta ${ }^{25 b}$, C.M. Gee ${ }^{143}$,

C.N.P. Gee ${ }^{141}$, J. Geisen ${ }^{94}$, M. Geisen ${ }^{97}$, C. Gemme ${ }^{53 b}$, M.H. Genest ${ }^{56}$, S. Gentile ${ }^{70 a, 70 b}$,

S. George ${ }^{91}$, T. Geralis ${ }^{42}$, L.O. Gerlach ${ }^{51}$, P. Gessinger-Befurt ${ }^{97}$, G. Gessner ${ }^{45}$,

M. Ghasemi Bostanabad ${ }^{173}$, M. Ghneimat ${ }^{149}$, A. Ghosh ${ }^{168}$, A. Ghosh ${ }^{75}$, B. Giacobbe ${ }^{21 b}$,

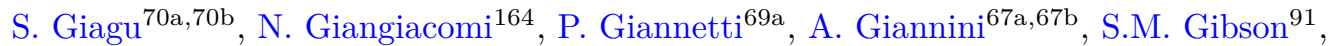

M. Gignac ${ }^{143}$, D.T. Gil ${ }^{81 b}$, B.J. Gilbert ${ }^{37}$, D. Gillberg ${ }^{32}$, G. Gilles ${ }^{179}$, N.E.K. Gillwald ${ }^{44}$,

D.M. Gingrich ${ }^{2, a k}$, M.P. Giordani ${ }^{64 a, 64 c}$, P.F. Giraud ${ }^{142}$, G. Giugliarelli ${ }^{64 a, 64 c}$, D. Giugni ${ }^{66 a}$,

F. Giuli ${ }^{71 a, 71 b}$, I. Gkialas ${ }^{8, i}$, E.L. Gkougkousis ${ }^{12}$, P. Gkountoumis ${ }^{9}$, L.K. Gladilin ${ }^{110}$,

C. Glasman ${ }^{96}$, G.R. Gledhill ${ }^{129}$, M. Glisic ${ }^{129}$, I. Gnesi ${ }^{39 b, d}$, M. Goblirsch-Kolb ${ }^{24}$, D. Godin ${ }^{107}$,

S. Goldfarb ${ }^{102}$, T. Golling ${ }^{52}$, D. Golubkov ${ }^{120}$, J.P. Gombas ${ }^{104}$, A. Gomes ${ }^{137 a, 137 b}$,

R. Goncalves Gama ${ }^{51}$, R. Gonçalo ${ }^{137 a, 137 c}$, G. Gonella ${ }^{129}$, L. Gonella ${ }^{19}$, A. Gongadze ${ }^{77}$,

F. Gonnella ${ }^{19}$, J.L. Gonski ${ }^{37}$, S. González de la Hoz ${ }^{171}$, S. Gonzalez Fernandez ${ }^{12}$,

R. Gonzalez Lopez ${ }^{88}$, C. Gonzalez Renteria ${ }^{16}$, R. Gonzalez Suarez ${ }^{169}$, S. Gonzalez-Sevilla ${ }^{52}$,

G.R. Gonzalvo Rodriguez ${ }^{171}$, R.Y. González Andana ${ }^{144 a}$, L. Goossens ${ }^{34}$, N.A. Gorasia ${ }^{19}$,

P.A. Gorbounov ${ }^{121}$, H.A. Gordon ${ }^{27}$, B. Gorini ${ }^{34}$, E. Gorini ${ }^{65 a, 65 b}$, A. Gorišek ${ }^{89}$, A.T. Goshaw ${ }^{47}$,

M.I. Gostkin ${ }^{77}$, C.A. Gottardo ${ }^{116}$, M. Gouighri ${ }^{33 b}$, V. Goumarre ${ }^{44}$, A.G. Goussiou ${ }^{146}$,

N. Govender ${ }^{31 c}$, C. Goy ${ }^{4}$, I. Grabowska-Bold ${ }^{81 a}$, K. Graham ${ }^{32}$, E. Gramstad ${ }^{131}$,

S. Grancagnolo ${ }^{17}$, M. Grandi ${ }^{154}$, V. Gratchev ${ }^{135}$, P.M. Gravila ${ }^{25 f}$, F.G. Gravili ${ }^{65 a, 65 b}$,

H.M. Gray ${ }^{16}$, C. Grefe ${ }^{22}$, I.M. Gregor ${ }^{44}$, P. Grenier ${ }^{151}$, K. Grevtsov ${ }^{44}$, C. Grieco ${ }^{12}$,

N.A. Grieser ${ }^{126}$, A.A. Grillo ${ }^{143}$, K. Grimm ${ }^{29, m}$, S. Grinstein ${ }^{12, x}$, J.-F. Grivaz ${ }^{62}$, S. Groh ${ }^{97}$,

E. Gross ${ }^{177}$, J. Grosse-Knetter ${ }^{51}$, Z.J. Grout ${ }^{92}$, C. Grud ${ }^{103}$, A. Grummer ${ }^{115}$, J.C. Grundy ${ }^{132}$,

L. Guan ${ }^{103}$, W. Guan ${ }^{178}$, C. Gubbels ${ }^{172}$, J. Guenther ${ }^{34}$, J.G.R. Guerrero Rojas ${ }^{171}$, F. Guescini ${ }^{112}$,

D. Guest ${ }^{17}$, R. Gugel ${ }^{97}$, A. Guida ${ }^{44}$, T. Guillemin ${ }^{4}$, S. Guindon ${ }^{34}$, J. Guo ${ }^{58 c}$, L. Guo ${ }^{62}$,

Y. Guo ${ }^{103}$, R. Gupta ${ }^{44}$, S. Gurbuz ${ }^{22}$, G. Gustavino ${ }^{126}$, M. Guth ${ }^{50}$, P. Gutierrez ${ }^{126}$,

L.F. Gutierrez Zagazeta ${ }^{134}$, C. Gutschow ${ }^{92}$, C. Guyot ${ }^{142}$, C. Gwenlan ${ }^{132}$, C.B. Gwilliam ${ }^{88}$,

E.S. Haaland ${ }^{131}$, A. Haas ${ }^{123}$, M. Habedank ${ }^{17}$, C. Haber ${ }^{16}$, H.K. Hadavand ${ }^{7}$, A. Hadef ${ }^{97}$,

M. Haleem ${ }^{174}$, J. Haley ${ }^{127}$, J.J. Hall ${ }^{147}$, G. Halladjian ${ }^{104}$, G.D. Hallewell ${ }^{99}$, L. Halser ${ }^{18}$,

K. Hamano ${ }^{173}$, H. Hamdaoui ${ }^{33 e}$, M. Hamer ${ }^{22}$, G.N. Hamity ${ }^{48}$, K. Han ${ }^{58 a}$, L. Han ${ }^{13 c}$, L. Han ${ }^{58 a}$,

S. $\operatorname{Han}^{16}$, Y.F. Han ${ }^{164}$, K. Hanagaki ${ }^{79, v}$, M. Hance ${ }^{143}$, M.D. Hank ${ }^{35}$, R. Hankache ${ }^{98}$, E. Hansen ${ }^{94}$, J.B. Hansen ${ }^{38}$, J.D. Hansen ${ }^{38}$, M.C. Hansen ${ }^{22}$, P.H. Hansen ${ }^{38}$, K. Hara ${ }^{166}$, T. Harenberg ${ }^{179}$,

S. Harkusha ${ }^{105}$, Y.T. Harris ${ }^{132}$, P.F. Harrison ${ }^{175}$, N.M. Hartman ${ }^{151}$, N.M. Hartmann ${ }^{111}$,

Y. Hasegawa ${ }^{148}$, A. Hasib ${ }^{48}$, S. Hassani ${ }^{142}$, S. Haug ${ }^{18}$, R. Hauser ${ }^{104}$, M. Havranek ${ }^{139}$,

C.M. Hawkes ${ }^{19}$, R.J. Hawkings ${ }^{34}$, S. Hayashida ${ }^{114}$, D. Hayden ${ }^{104}$, C. Hayes ${ }^{103}$, R.L. Hayes ${ }^{172}$,

C.P. Hays ${ }^{132}$, J.M. Hays ${ }^{90}$, H.S. Hayward ${ }^{88}$, S.J. Haywood ${ }^{141}$, F. He ${ }^{58 a}$, Y. He ${ }^{162}$, Y. He$^{133}$,

M.P. Heath ${ }^{48}$, V. Hedberg ${ }^{94}$, A.L. Heggelund ${ }^{131}$, N.D. Hehir ${ }^{90}$, C. Heidegger ${ }^{50}$, K.K. Heidegger ${ }^{50}$,

W.D. Heidorn ${ }^{76}$, J. Heilman ${ }^{32}$, S. Heim ${ }^{44}$, T. Heim ${ }^{16}$, B. Heinemann ${ }^{44, a i}$, J.G. Heinlein ${ }^{134}$,

J.J. Heinrich ${ }^{129}$, L. Heinrich ${ }^{34}$, J. Hejbal ${ }^{138}$, L. Helary ${ }^{44}$, A. Held ${ }^{123}$, S. Hellesund ${ }^{131}$,

C.M. Helling ${ }^{143}$, S. Hellman ${ }^{43 a}$,43b , C. Helsens ${ }^{34}$, R.C.W. Henderson ${ }^{87}$, L. Henkelmann ${ }^{30}$,

A.M. Henriques Correia ${ }^{34}$, H. Herde ${ }^{151}$, Y. Hernández Jiménez ${ }^{31 f}$, H. Herr ${ }^{97}$, M.G. Herrmann ${ }^{111}$,

T. Herrmann ${ }^{46}$, G. Herten ${ }^{50}$, R. Hertenberger ${ }^{111}$, L. Hervas ${ }^{34}$, N.P. Hessey ${ }^{165 a}$, H. Hibi ${ }^{80}$,

S. Higashino ${ }^{79}$, E. Higón-Rodriguez ${ }^{171}$, K.K. Hill ${ }^{27}$, K.H. Hiller ${ }^{44}$, S.J. Hillier ${ }^{19}$, M. Hils ${ }^{46}$, 
I. Hinchliffe $\mathrm{e}^{16}$, F. Hinterkeuser ${ }^{22}$, M. Hirose ${ }^{130}$, S. Hirose ${ }^{166}$, D. Hirschbuehl ${ }^{179}$, B. Hiti ${ }^{89}$, O. Hladik ${ }^{138}$, J. Hobbs ${ }^{153}$, R. Hobincu ${ }^{25 e}$, N. Hod ${ }^{177}$, M.C. Hodgkinson ${ }^{147}$, B.H. Hodkinson ${ }^{30}$, A. Hoecker ${ }^{34}$, J. Hofer ${ }^{44}$, D. Hohn ${ }^{50}$, T. Holm ${ }^{22}$, T.R. Holmes ${ }^{35}$, M. Holzbock ${ }^{112}$, L.B.A.H. Hommels ${ }^{30}$, B.P. Honan ${ }^{98}$, T.M. Hong ${ }^{136}$, J.C. Honig ${ }^{50}$, A. Hönle ${ }^{112}$, B.H. Hooberman ${ }^{170}$, W.H. Hopkins ${ }^{5}$, Y. Horii ${ }^{114}$, P. Horn ${ }^{46}$, L.A. Horyn ${ }^{35}$, S. Hou ${ }^{156}$, J. Howarth ${ }^{55}$, J. Hoya ${ }^{86}$, M. Hrabovsky ${ }^{128}$, A. Hrynevich ${ }^{106}$, T. Hryn'ova ${ }^{4}$, P.J. Hsu ${ }^{61}$, S.-C. $\mathrm{Hsu}^{146}$, Q. Hu ${ }^{37}$, S. Hu ${ }^{58 c}$, Y.F. Hu${ }^{13 a, 13 d, a m}$, D.P. Huang ${ }^{92}$, X. Huang ${ }^{13 c}$, Y. Huang ${ }^{58 a}$, Y. Huang ${ }^{13 a}$, Z. Hubacek ${ }^{139}$, F. Hubaut ${ }^{99}$, M. Huebner ${ }^{22}$, F. Huegging ${ }^{22}$, T.B. Huffman ${ }^{132}$, M. Huhtinen ${ }^{34}$, R. Hulsken ${ }^{56}$, N. Huseynov ${ }^{77, a b}$, J. Huston ${ }^{104}$, J. Huth ${ }^{57}$, R. Hyneman ${ }^{151}$, S. Hyrych ${ }^{26 a}$, G. Iacobucci ${ }^{52}$, G. Iakovidis ${ }^{27}$, I. Ibragimov ${ }^{149}$, L. Iconomidou-Fayard ${ }^{62}$, P. Iengo ${ }^{34}$,

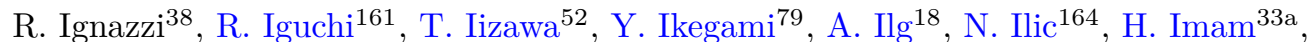

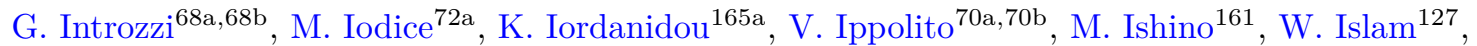

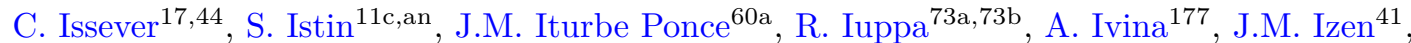
V. Izzo ${ }^{67 a}$, P. Jacka ${ }^{138}$, P. Jackson ${ }^{1}$, R.M. Jacobs ${ }^{44}$, B.P. Jaeger ${ }^{150}$, C.S. Jagfeld ${ }^{111}$, G. Jäkel ${ }^{179}$, K.B. Jakobi ${ }^{97}$, K. Jakobs ${ }^{50}$, T. Jakoubek ${ }^{177}$, J. Jamieson ${ }^{55}$, K.W. Janas ${ }^{81 a}$, G. Jarlskog ${ }^{94}$, A.E. Jaspan ${ }^{88}$, N. Javadov ${ }^{77, a b}$, T. Javůrek ${ }^{34}$, M. Javurkova ${ }^{100}$, F. Jeanneau ${ }^{142}$, L. Jeanty ${ }^{129}$, J. Jejelava ${ }^{157 a, a c}$, P. Jenni ${ }^{50, e}$, S. Jézéquel ${ }^{4}$, J. Jia ${ }^{153}$, Z. Jia ${ }^{13 c}$, Y. Jiang ${ }^{58 a}$, S. Jiggins ${ }^{50}$, J. Jimenez Pena ${ }^{112}$, S. Jin ${ }^{13 c}$, A. Jinaru ${ }^{25 b}$, O. Jinnouchi ${ }^{162}$, H. Jivan ${ }^{31 f}$, P. Johansson ${ }^{147}$, K.A. Johns ${ }^{6}$, C.A. Johnson ${ }^{63}$, E. Jones ${ }^{175}$, R.W.L. Jones ${ }^{87}$, T.J. Jones ${ }^{88}$, J. Jovicevic ${ }^{34}$, X. Ju ${ }^{16}$, J.J. Junggeburth ${ }^{112}$, A. Juste Rozas ${ }^{12, x}$, A. Kaczmarska ${ }^{82}$, M. Kado ${ }^{70 a, 70 b}$, H. Kagan ${ }^{125}$, M. Kagan ${ }^{151}$, A. Kahn ${ }^{37}$, C. Kahra ${ }^{97}$, T. Kaji ${ }^{176}$, E. Kajomovitz ${ }^{158}$, C.W. Kalderon ${ }^{27}$, A. Kaluza ${ }^{97}$, A. Kamenshchikov ${ }^{120}$, M. Kaneda ${ }^{161}$, N.J. Kang ${ }^{143}$, S. Kang ${ }^{76}$, Y. Kano ${ }^{114}$, J. Kanzaki ${ }^{79}$, D. Kar ${ }^{31 f}$, K. Karava ${ }^{132}$, M.J. Kareem ${ }^{165 b}$, I. Karkanias ${ }^{160}$, S.N. Karpov ${ }^{77}$, Z.M. Karpova ${ }^{77}$, V. Kartvelishvili ${ }^{87}$, A.N. Karyukhin ${ }^{120}$, E. Kasimi ${ }^{160}$, C. Kato ${ }^{58 d}$, J. Katzy ${ }^{44}$, K. Kawade ${ }^{148}$, K. Kawagoe ${ }^{85}$, T. Kawaguchi ${ }^{114}$, T. Kawamoto ${ }^{142}$, G. Kawamura ${ }^{51}$, E.F. Kay ${ }^{173}$, F.I. Kaya ${ }^{167}$, S. Kazakos ${ }^{12}$, V.F. Kazanin ${ }^{119 b, 119 a}$, Y. Ke ${ }^{153}$, J.M. Keaveney ${ }^{31 a}$, R. Keeler ${ }^{173}$, J.S. Keller ${ }^{32}$, D. Kelsey ${ }^{154}$, J.J. Kempster ${ }^{19}$, J. Kendrick ${ }^{19}$, K.E. Kennedy ${ }^{37}$, O. Kepka ${ }^{138}$, S. Kersten ${ }^{179}$, B.P. Kerševan ${ }^{89}$, S. Ketabchi Haghighat ${ }^{164}$, M. Khandoga ${ }^{133}$, A. Khanov ${ }^{127}$, A.G. Kharlamov ${ }^{119 b, 119 a}$, T. Kharlamova119b,119a, E.E. Khoda ${ }^{172}$, T.J. Khoo ${ }^{17}$, G. Khoriauli ${ }^{174}$, E. Khramov ${ }^{77}$, J. Khubua ${ }^{157 b}$, S. Kido ${ }^{80}$, M. Kiehn ${ }^{34}$, A. Kilgallon ${ }^{129}$, E. Kim ${ }^{162}$, Y.K. Kim ${ }^{35}$, N. Kimura ${ }^{92}$, A. Kirchhoff ${ }^{51}$, D. Kirchmeier ${ }^{46}$, J. Kirk ${ }^{141}$, A.E. Kiryunin ${ }^{112}$, T. Kishimoto ${ }^{161}$, D.P. Kisliuk ${ }^{164}$, V. Kitali ${ }^{44}$, C. Kitsaki ${ }^{9}$, O. Kivernyk ${ }^{22}$, T. Klapdor-Kleingrothaus ${ }^{50}$, M. Klassen ${ }^{59 a}$, C. Klein ${ }^{32}$, L. Klein ${ }^{174}$, M.H. Klein ${ }^{103}$, M. Klein ${ }^{88}$, U. Klein ${ }^{88}$, P. Klimek ${ }^{34}$, A. Klimentov ${ }^{27}$, F. Klimpel ${ }^{34}$, T. Klingl ${ }^{22}$, T. Klioutchnikova ${ }^{34}$, F.F. Klitzner ${ }^{111}$, P. Kluit ${ }^{117}$, S. Kluth ${ }^{112}$, E. Kneringer ${ }^{74}$, T.M. Knight ${ }^{164}$, A. Knue ${ }^{50}$, D. Kobayashi ${ }^{85}$, M. Kobel ${ }^{46}$, M. Kocian ${ }^{151}$, T. Kodama ${ }^{161}$, P. Kodys ${ }^{140}$, D.M. Koeck ${ }^{154}$, P.T. Koenig ${ }^{22}$, T. Koffas ${ }^{32}$, N.M. Köhler ${ }^{34}$, M. Kolb ${ }^{142}$, I. Koletsou ${ }^{4}$, T. Komarek ${ }^{128}$, K. Köneke ${ }^{50}$, A.X.Y. Kong ${ }^{1}$, T. Kono ${ }^{124}$, V. Konstantinides ${ }^{92}$, N. Konstantinidis ${ }^{92}$, B. Konya ${ }^{94}$, R. Kopeliansky ${ }^{63}$, S. Koperny ${ }^{81 a}$, K. Korcyl ${ }^{82}$, K. Kordas ${ }^{160}$, G. Koren ${ }^{159}$, A. Korn ${ }^{92}$, S. Korn ${ }^{51}$, I. Korolkov ${ }^{12}$, E.V. Korolkova ${ }^{147}$, N. Korotkova ${ }^{110}$, O. Kortner ${ }^{112}$, S. Kortner ${ }^{112}$, V.V. Kostyukhin ${ }^{147,163}$, A. Kotsokechagia ${ }^{62}$, A. Kotwal ${ }^{47}$, A. Koulouris ${ }^{8}$, A. Kourkoumeli-Charalampidi ${ }^{68 a, 68 b,}$ C. Kourkoumelis ${ }^{8}$, E. Kourlitis ${ }^{5}$, R. Kowalewski ${ }^{173}$, W. Kozanecki ${ }^{142}$, A.S. Kozhin ${ }^{120}$, V.A. Kramarenko ${ }^{110}$, G. Kramberger ${ }^{89}$, D. Krasnopevtsev ${ }^{58 a}$, M.W. Krasny ${ }^{133}$,

A. Krasznahorkay ${ }^{34}$, J.A. Kremer ${ }^{97}$, J. Kretzschmar ${ }^{88}$, K. Kreul ${ }^{17}$, P. Krieger ${ }^{164}$, F. Krieter ${ }^{111}$, S. Krishnamurthy ${ }^{100}$, A. Krishnan ${ }^{59 b}$, M. Krivos ${ }^{140}$, K. Krizka ${ }^{16}$, K. Kroeninger ${ }^{45}$, H. Kroha ${ }^{112}$, J. Kroll ${ }^{138}$, J. Kroll ${ }^{134}$, K.S. Krowpman ${ }^{104}$, U. Kruchonak ${ }^{77}$, H. Krüger ${ }^{22}$, N. Krumnack ${ }^{76}$, M.C. Kruse ${ }^{47}$, J.A. Krzysiak ${ }^{82}$, A. Kubota ${ }^{162}$, O. Kuchinskaia ${ }^{163}$, S. Kuday ${ }^{3 b}$, D. Kuechler ${ }^{44}$, J.T. Kuechler ${ }^{44}$, S. Kuehn ${ }^{34}$, T. Kuhl ${ }^{44}$, V. Kukhtin ${ }^{77}$, Y. Kulchitsky ${ }^{105, \text { af }}$, S. Kuleshov ${ }^{144 d}$, 
M. Kumar ${ }^{31 f}$, N. Kumari ${ }^{99}$, M. Kuna ${ }^{56}$, A. Kupco ${ }^{138}$, T. Kupfer ${ }^{45}$, O. Kuprash $^{50}$, H. Kurashige ${ }^{80}$, L.L. Kurchaninov ${ }^{165 a}$, Y.A. Kurochkin ${ }^{105}$, A. Kurova ${ }^{109}$, M.G. Kurth ${ }^{13 a, 13 d}$, E.S. Kuwertz ${ }^{34}$, M. Kuze ${ }^{162}$, A.K. Kvam ${ }^{146}$, J. Kvita ${ }^{128}$, T. Kwan ${ }^{101}$, C. Lacasta ${ }^{171}$, F. Lacava ${ }^{70 a}$,70b, H. Lacker ${ }^{17}$, D. Lacour ${ }^{133}$, E. Ladygin ${ }^{77}$, R. Lafaye ${ }^{4}$, B. Laforge ${ }^{133}$, T. Lagouri ${ }^{144 e}$, S. Lai ${ }^{51}$, I.K. Lakomiec ${ }^{81 a}$, N. Lalloue ${ }^{56}$, J.E. Lambert ${ }^{126}$, S. Lammers ${ }^{63}$, W. Lampl ${ }^{6}$, C. Lampoudis ${ }^{160}$, E. Lançon ${ }^{27}$, U. Landgraf ${ }^{50}$, M.P.J. Landon ${ }^{90}$, V.S. Lang ${ }^{50}$, J.C. Lange ${ }^{51}$, R.J. Langenberg ${ }^{100}$, A.J. Lankford ${ }^{168}$, F. Lanni ${ }^{27}$, K. Lantzsch ${ }^{22}$, A. Lanza ${ }^{68 a}$, A. Lapertosa ${ }^{53 b, 53 a}$, J.F. Laporte ${ }^{142}$, T. Lari ${ }^{66 a}$, F. Lasagni Manghi ${ }^{21 b}$, M. Lassnig ${ }^{34}$, V. Latonova ${ }^{138}$, T.S. Lau ${ }^{60 a}$, A. Laudrain ${ }^{97}$, A. Laurier ${ }^{32}$, M. Lavorgna ${ }^{67 a, 67 b}$, S.D. Lawlor ${ }^{91}$, M. Lazzaroni66a,66b ${ }^{6}$ B. Le ${ }^{98}$, A. Lebedev ${ }^{76}$, M. LeBlanc ${ }^{34}$, T. LeCompte ${ }^{5}$, F. Ledroit-Guillon ${ }^{56}$, A.C.A. Lee ${ }^{92}$, C.A. Lee $^{27}$, G.R. Lee ${ }^{15}$, L. Lee ${ }^{57}$, S.C. Lee ${ }^{156}$, S. Lee ${ }^{76}$, L.L. Leeuw ${ }^{31 c}$, B. Lefebvre ${ }^{165 a}$, H.P. Lefebvre ${ }^{91}$, M. Lefebvre ${ }^{173}$, C. Leggett ${ }^{16}$, K. Lehmann ${ }^{150}$, N. Lehmann ${ }^{18}$, G. Lehmann Miotto ${ }^{34}$, W.A. Leight ${ }^{44}$, A. Leisos ${ }^{160, w}$, M.A.L. Leite ${ }^{78 c}$, C.E. Leitgeb ${ }^{44}$, R. Leitner ${ }^{140}$, K.J.C. Leney ${ }^{40}$, T. Lenz ${ }^{22}$, S. Leone ${ }^{69 a}$, C. Leonidopoulos ${ }^{48}$, A. Leopold ${ }^{133}$, C. Leroy ${ }^{107}$, R. Les ${ }^{104}$, C.G. Lester ${ }^{30}$, M. Levchenko ${ }^{135}$, J. Levêque ${ }^{4}$, D. Levin ${ }^{103}$, L.J. Levinson ${ }^{177}$, D.J. Lewis ${ }^{19}$, B. Li ${ }^{13 b}$, B. Li $^{103}$,

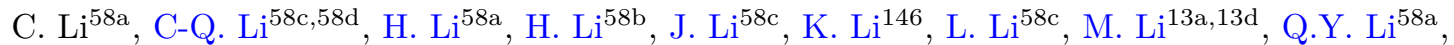
S. Li ${ }^{58 \mathrm{~d}, 58 \mathrm{c}, \mathrm{c}}, \mathrm{X} . \mathrm{Li}^{44}, \mathrm{Y} . \mathrm{Li}^{44}$, Z. Li ${ }^{58 \mathrm{~b}}$, Z. $\mathrm{Li}^{132}$, Z. $\mathrm{Li}^{101}$, Z. Li ${ }^{88}$, Z. Liang ${ }^{13 a}$, M. Liberatore ${ }^{44}$,

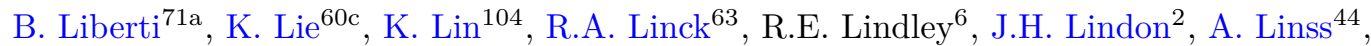
A.L. Lionti ${ }^{52}$, E. Lipeles ${ }^{134}$, A. Lipniacka ${ }^{15}$, T.M. Liss ${ }^{170, a j}$, A. Lister ${ }^{172}$, J.D. Little ${ }^{7}$, B. Liu ${ }^{13 a}$,

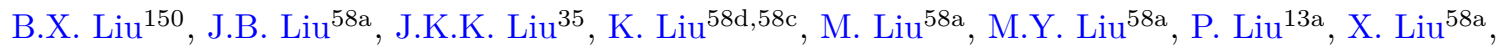

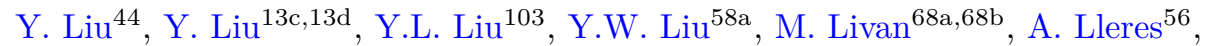

J. Llorente Merino ${ }^{150}$, S.L. Lloyd ${ }^{90}$, E.M. Lobodzinska ${ }^{44}$, P. Loch $^{6}$, S. Loffredo ${ }^{71 a, 71 b}$, T. Lohse ${ }^{17}$, K. Lohwasser ${ }^{147}$, M. Lokajicek ${ }^{138}$, J.D. Long ${ }^{170}$, R.E. Long ${ }^{87}$, I. Longarini ${ }^{70 a, 70 b}$, L. Longo ${ }^{34}$, R. Longo ${ }^{170}$, I. Lopez Paz ${ }^{12}$, A. Lopez Solis ${ }^{44}$, J. Lorenz ${ }^{111}$, N. Lorenzo Martinez ${ }^{4}$, A.M. Lory ${ }^{111}$, A. Lösle ${ }^{50}$, X. Lou ${ }^{43 a, 43 b}$, X. Lou ${ }^{13 a}$, A. Lounis ${ }^{62}$, J. Love ${ }^{5}$, P.A. Love ${ }^{87}$, J.J. Lozano Bahilo ${ }^{171}$, G. $\mathrm{Lu}^{13 \mathrm{a}}, \mathrm{M} . \mathrm{Lu}^{58 \mathrm{a}}$, S. $\mathrm{Lu}^{134}$, Y.J. Lu ${ }^{61}$, H.J. Lubatti ${ }^{146}$, C. Luci ${ }^{70 a}$,70b, F.L. Lucio Alves ${ }^{13 c}$, A. Lucotte $^{56}$, F. Luehring ${ }^{63}$, I. Luise ${ }^{153}$, L. Luminari ${ }^{70 a}$, B. Lund-Jensen ${ }^{152}$, N.A. Luongo ${ }^{129}$, M.S. Lutz ${ }^{159}$, D. Lynn ${ }^{27}$, H. Lyons ${ }^{88}$, R. Lysak ${ }^{138}$, E. Lytken ${ }^{94}$, F. Lyu ${ }^{13 a}$, V. Lyubushkin ${ }^{77}$, T. Lyubushkina ${ }^{77}$, H. $\mathrm{Ma}^{27}$, L.L. Ma ${ }^{58 b}$, Y. Ma ${ }^{92}$, D.M. Mac Donell ${ }^{173}$, G. Maccarrone ${ }^{49}$, C.M. Macdonald ${ }^{147}$, J.C. MacDonald ${ }^{147}$, R. Madar ${ }^{36}$, W.F. Mader ${ }^{46}$,

M. Madugoda Ralalage Don ${ }^{127}$, N. Madysa ${ }^{46}$, J. Maeda ${ }^{80}$, T. Maeno ${ }^{27}$, M. Maerker ${ }^{46}$, V. Magerl ${ }^{50}$, J. Magro ${ }^{64 a, 64 c}$, D.J. Mahon ${ }^{37}$, C. Maidantchik ${ }^{78 b}$, A. Maio ${ }^{137 a, 137 b, 137 d}$, K. Maj81a, O. Majersky ${ }^{26 a}$, S. Majewskii ${ }^{129}$, N. Makovec ${ }^{62}$, B. Malaescu ${ }^{133}$, Pa. Malecki ${ }^{82}$, V.P. Maleev ${ }^{135}$, F. Malek ${ }^{56}$, D. Malito ${ }^{39 b, 39 a}$, U. Mallik ${ }^{75}$, C. Malone ${ }^{30}$, S. Maltezos $^{9}$, S. Malyukov ${ }^{77}$, J. Mamuzic ${ }^{171}$, G. Mancini ${ }^{49}$, J.P. Mandalia ${ }^{90}$, I. Mandićc ${ }^{89}$, L. Manhaes de Andrade Filho ${ }^{78 a}$, I.M. Maniatis ${ }^{160}$, M. Manisha ${ }^{142}$, J. Manjarres Ramos ${ }^{46}$, K.H. Mankinen ${ }^{94}$, A. Mann ${ }^{111}$, A. Manousos ${ }^{74}$, B. Mansoulie ${ }^{142}$, I. Manthos ${ }^{160}$, S. Manzoni ${ }^{117}$, A. Marantis ${ }^{160, w}$, L. Marchese ${ }^{132}$, G. Marchiori ${ }^{133}$, M. Marcisovsky ${ }^{138}$, L. Marcoccia ${ }^{71 a, 71 b}$, C. Marcon ${ }^{94}$, M. Marjanovic ${ }^{126}$, Z. Marshall ${ }^{16}$, S. Marti-Garcia ${ }^{171}$, T.A. Martin ${ }^{175}$, V.J. Martin ${ }^{48}$, B. Martin dit Latour ${ }^{15}$, L. Martinelli ${ }^{72 a, 72 b}$, M. Martinez ${ }^{12, x}$, P. Martinez Agullo ${ }^{171}$, V.I. Martinez Outschoorn ${ }^{100}$, S. Martin-Haugh ${ }^{141}$, V.S. Martoiu ${ }^{25 b}$, A.C. Martyniuk ${ }^{92}$, A. Marzin ${ }^{34}$, S.R. Maschek ${ }^{112}$, L. Masetti ${ }^{97}$, T. Mashimo ${ }^{161}$, R. Mashinistov ${ }^{108}$, J. Masik ${ }^{98}$, A.L. Maslennikov ${ }^{119 b, 119 a}$, L. Massa ${ }^{21 b}$, P. Massarotti ${ }^{67 a, 67 b}$, P. Mastrandrea ${ }^{69 a, 69 b}$, A. Mastroberardino ${ }^{39 b, 39 a}$, T. Masubuchi ${ }^{161}$, D. Matakias ${ }^{27}$, T. Mathisen ${ }^{169}$, A. Matic ${ }^{111}$, N. Matsuzawa ${ }^{161}$, J. Maurer ${ }^{25 b}$, B. Maček ${ }^{89}$, D.A. Maximov ${ }^{119 b, 119 a}$, R. Mazini1 ${ }^{156}$, I. Maznas ${ }^{160}$, S.M. Mazza ${ }^{143}$, C. Mc Ginn ${ }^{27}$, J.P. Mc Gowan ${ }^{101}$, S.P. Mc Kee ${ }^{103}$, T.G. McCarthy ${ }^{112}$, W.P. McCormack ${ }^{16}$, E.F. McDonald ${ }^{102}$, A.E. McDougall ${ }^{117}$, J.A. Mcfayden ${ }^{154}$, G. Mchedlidze ${ }^{157 b}$, M.A. McKay ${ }^{40}$, K.D. McLean ${ }^{173}$, S.J. McMahon ${ }^{141}$, P.C. McNamara ${ }^{102}$, R.A. McPherson ${ }^{173, \text { aa }}$, J.E. Mdhluliifif, Z.A. Meadows ${ }^{100}$, 


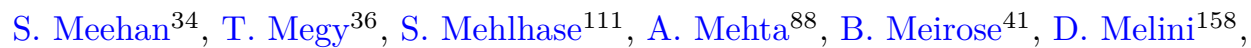
B.R. Mellado Garcia ${ }^{31 f}$, F. Meloni ${ }^{44}$, A. Melzer ${ }^{22}$, E.D. Mendes Gouveia ${ }^{137 a}$, A.M. Mendes Jacques Da Costa ${ }^{19}$, H.Y. Meng ${ }^{164}$, L. Meng ${ }^{34}$, S. Menke ${ }^{112}$, M. Mentink ${ }^{34}$, E. Meoni ${ }^{39 b, 39 a}$, S.A.M. Merkt ${ }^{136}$, C. Merlassino ${ }^{132}$, P. Mermod ${ }^{52, *}$, L. Merola ${ }^{67 a, 67 b}$, C. Meroni ${ }^{66 a}$, G. Merz ${ }^{103}$, O. Meshkov ${ }^{110,108}$, J.K.R. Meshreki ${ }^{149}$, J. Metcalfe ${ }^{5}$, A.S. Mete ${ }^{5}$, C. Meyer ${ }^{63}$, J-P. Meyer ${ }^{142}$, M. Michetti ${ }^{17}$, R.P. Middleton ${ }^{141}$, L. Mijović ${ }^{48}$, G. Mikenberg ${ }^{177}$, M. Mikestikova ${ }^{138}$, M. Mikuž ${ }^{89}$, H. Mildner ${ }^{147}$, A. Milic ${ }^{164}$, C.D. Milke ${ }^{40}$, D.W. Miller ${ }^{35}$, L.S. Miller ${ }^{32}$, A. Milov ${ }^{177}$, D.A. Milstead ${ }^{43 a, 43 b}$, A.A. Minaenko ${ }^{120}$, I.A. Minashvili ${ }^{157 b}$, L. Mince ${ }^{55}$, A.I. Mincer ${ }^{123}$, B. Mindur ${ }^{81 a}$, M. Mineev ${ }^{77}$, Y. Minegishi ${ }^{161}$, Y. Mino ${ }^{83}$, L.M. Mir ${ }^{12}$, M. Miralles Lopez ${ }^{171}$, M. Mironova ${ }^{132}$, T. Mitani ${ }^{176}$, V.A. Mitsou ${ }^{171}$, M. Mittal ${ }^{58 c}$, O. Miu ${ }^{164}$, P.S. Miyagawa ${ }^{90}$, Y. Miyazaki ${ }^{85}$, A. Mizukami ${ }^{79}$, J.U. Mjörnmark ${ }^{94}$, T. Mkrtchyan ${ }^{59 a}$, M. Mlynarikova ${ }^{118}$, T. Moa ${ }^{43 a}$,43b, S. Mobius ${ }^{51}$, K. Mochizuki ${ }^{107}$, P. Moder ${ }^{44}$, P. Mogg ${ }^{111}$, S. Mohapatra ${ }^{37}$, G. Mokgatitswane ${ }^{31 f}$, B. Mondal ${ }^{149}$, S. Mondal ${ }^{139}$, K. Mönig ${ }^{44}$, E. Monnier ${ }^{99}$, A. Montalbano ${ }^{150}$, J. Montejo Berlingen ${ }^{34}$, M. Montella ${ }^{125}$, F. Monticelli ${ }^{86}$, N. Morange ${ }^{62}$, A.L. Moreira De Carvalho ${ }^{137 a}$, M. Moreno Llácer ${ }^{171}$, C. Moreno Martinez ${ }^{12}$, P. Morettini ${ }^{53 b}$, M. Morgenstern ${ }^{158}$, S. Morgenstern ${ }^{175}$, D. Mori ${ }^{150}$, M. Morii ${ }^{57}$, M. Morinaga ${ }^{176}$, V. Morisbak ${ }^{131}$, A.K. Morley ${ }^{34}$, A.P. Morris ${ }^{92}$, L. Morvaj ${ }^{34}$, P. Moschovakos ${ }^{34}$, B. Moser ${ }^{117}$, M. Mosidze ${ }^{157 b}$, T. Moskalets ${ }^{50}$, P. Moskvitina ${ }^{116}$, J. Moss ${ }^{29, o}$, E.J.W. Moyse ${ }^{100}$, S. Muanza ${ }^{99}$, J. Mueller ${ }^{136}$, D. Muenstermann ${ }^{87}$, G.A. Mullier ${ }^{94}$, J.J. Mullin ${ }^{134}$, D.P. Mungo ${ }^{66 a, 66 b}$, J.L. Munoz Martinez ${ }^{12}$, F.J. Munoz Sanchez ${ }^{98}$, M. Murin ${ }^{98}$, P. Murinn ${ }^{26 b}$, W.J. Murray ${ }^{175,141}$, A. Murrone ${ }^{66 a, 66 b}$, J.M. Muse ${ }^{126}$, M. Muškinja ${ }^{16}$, C. Mwewa ${ }^{27}$, A.G. Myagkov ${ }^{120, a g}$, A.A. Myers ${ }^{136}$, G. Myers ${ }^{63}$, J. Myers ${ }^{129}$, M. Myska ${ }^{139}$, B.P. Nachman ${ }^{16}$, O. Nackenhorst ${ }^{45}$, A.Nag Nag ${ }^{46}$, K. Nagai ${ }^{132}$, K. Nagano ${ }^{79}$, J.L. Nagle ${ }^{27}$, E. Nagy ${ }^{99}$, A.M. Nairz ${ }^{34}$, Y. Nakahama ${ }^{114}$, K. Nakamura ${ }^{79}$, H. Nanjo ${ }^{130}$, F. Napolitano ${ }^{59 a}$, R.F. Naranjo Garcia ${ }^{44}$, R. Narayan ${ }^{40}$, I. Naryshkin ${ }^{135}$,

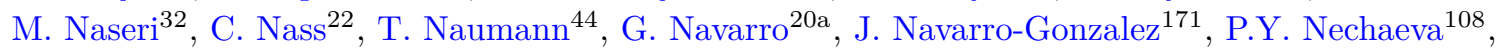
F. Nechansky ${ }^{44}$, T.J. Neep ${ }^{19}$, A. Negri ${ }^{68 a, 68 b}$, M. Negrini ${ }^{21 b}$, C. Nellist ${ }^{116}$, C. Nelson ${ }^{101}$, K. Nelson ${ }^{103}$, M.E. Nelson ${ }^{43 a, 43 b}$, S. Nemecek ${ }^{138}$, M. Nessi ${ }^{34, g}$, M.S. Neubauer ${ }^{170}$, F. Neuhaus ${ }^{97}$, M. Neumann ${ }^{179}$, R. Newhouse ${ }^{172}$, P.R. Newman ${ }^{19}$, C.W. $\mathrm{Ng}^{136}$, Y.S. $\mathrm{Ng}^{17}$, Y.W.Y. Ng${ }^{168}$, B. Ngair ${ }^{33 e}$, H.D.N. Nguyen ${ }^{99}$, T. Nguyen Manh ${ }^{107}$, R.B. Nickerson ${ }^{132}$, R. Nicolaidou ${ }^{142}$, D.S. Nielsen ${ }^{38}$, J. Nielsen ${ }^{143}$, M. Niemeyer ${ }^{51}$, N. Nikiforou ${ }^{10}$, V. Nikolaenko ${ }^{120, a g}$, I. Nikolic-Audit ${ }^{133}$, K. Nikolopoulos ${ }^{19}$, P. Nilsson ${ }^{27}$, H.R. Nindhito ${ }^{52}$, A. Nisati ${ }^{70 a}$, N. Nishu ${ }^{2}$, R. Nisius ${ }^{112}$, T. Nitta ${ }^{176}$, T. Nobe ${ }^{161}$, D.L. Noel $^{30}$, Y. $\operatorname{Noguchi}^{83}$, I. Nomidis ${ }^{133}$, M.A. Nomura ${ }^{27}$, M.B. Norfolk ${ }^{147}$, R.R.B. Norisam ${ }^{92}$, J. Novak ${ }^{89}$, T. Novak $^{44}$, O. Novgorodova ${ }^{46}$, L. Novotny ${ }^{139}$,

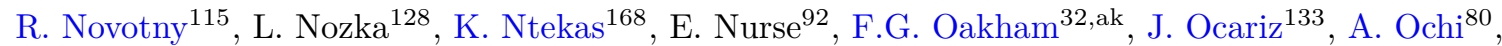
I. Ochoa ${ }^{137 a}$, J.P. Ochoa-Ricoux ${ }^{144 a}$, K. O'Connor ${ }^{24}$, S. Oda ${ }^{85}$, S. Odaka ${ }^{79}$, S. Oerdek $^{51}$, A. Ogrodnik ${ }^{81 a}$, A. $\mathrm{Oh}^{98}$, C.C. Ohm ${ }^{152}$, H. Oide ${ }^{162}$, R. Oishi ${ }^{161}$, M.L. Ojeda ${ }^{164}$, Y. Okazaki ${ }^{83}$, M.W. O'Keefe ${ }^{88}$, Y. Okumura ${ }^{161}$, A. Olariu ${ }^{25 b}$, L.F. Oleiro Seabra ${ }^{137 a}$, S.A. Olivares Pino ${ }^{144 e}$, D. Oliveira Damazio ${ }^{27}$, D. Oliveira Goncalves ${ }^{78 a}$, J.L. Oliver ${ }^{1}$, M.J.R. Olsson ${ }^{168}$, A. Olszewski ${ }^{82}$, J. Olszowska ${ }^{82}$, Ö.O. Öncel ${ }^{22}$, D.C. O'Neil ${ }^{150}$, A.P. O'neill ${ }^{132}$, A. Onofre ${ }^{137 a}, 137$, P.U.E. Onyisi ${ }^{10}$, H. Oppen ${ }^{131}$, R.G. Oreamuno Madriz ${ }^{118}$, M.J. Oreglia ${ }^{35}$, G.E. Orellana ${ }^{86}$, D. Orestano ${ }^{72 a, 72 b}$, N. Orlando ${ }^{12}$, R.S. Orr ${ }^{164}$, V. O'Shea ${ }^{55}$, R. Ospanov ${ }^{58 a}$, G. Otero y Garzon ${ }^{28}$, H. Otono ${ }^{85}$, P.S. Ott ${ }^{59 a}$, G.J. Ottino ${ }^{16}$, M. Ouchrif ${ }^{33 d}$, J. Ouellette ${ }^{27}$, F. Ould-Saada ${ }^{131}$, A. Ouraou ${ }^{142, *}$, Q. Ouyang ${ }^{13 a}$, M. Owen ${ }^{55}$, R.E. Owen ${ }^{141}$, V.E. Ozcan ${ }^{11 c}$, N. Ozturk ${ }^{7}$, J. Pacalt ${ }^{128}$, H.A. Pacey ${ }^{30}$, K. Pachal ${ }^{47}$, A. Pacheco Pages ${ }^{12}$, C. Padilla Aranda ${ }^{12}$, S. Pagan Griso ${ }^{16}$, G. Palacino ${ }^{63}$, S. Palazzo ${ }^{48}$, S. Palestini ${ }^{34}$, M. Palka ${ }^{81 b}$, P. Palni ${ }^{81 a}$, D.K. Panchal ${ }^{10}$, C.E. Pandini ${ }^{52}$, J.G. Panduro Vazquez ${ }^{91}$, P. Pani ${ }^{44}$, G. Panizzo ${ }^{64 a, 64 c}$, L. Paolozzi ${ }^{52}$, C. Papadatos ${ }^{107}$, S. Parajuli ${ }^{40}$, A. Paramonov ${ }^{5}$, C. Paraskevopoulos ${ }^{9}$, D. Paredes Hernandez ${ }^{60 b}$, S.R. Paredes Saenz ${ }^{132}$, B. Parida ${ }^{177}$, T.H. Park ${ }^{164}$, A.J. Parker ${ }^{29}$, M.A. Parker ${ }^{30}$, F. Parodi ${ }^{53 b, 53 a,}$ 
E.W. Parrish ${ }^{118}$, J.A. Parsons ${ }^{37}$, U. Parzefall ${ }^{50}$, L. Pascual Dominguez ${ }^{133}$, V.R. Pascuzzi ${ }^{16}$, F. Pasquali ${ }^{117}$, E. Pasqualucci ${ }^{70 a}$, S. Passaggio ${ }^{53 b}$, F. Pastore ${ }^{91}$, P. Pasuwan ${ }^{43 a}, 43 b$, J.R. Pater ${ }^{98}$, A. Pathak ${ }^{178, k}$, J. Patton ${ }^{88}$, T. Pauly ${ }^{34}$, J. Pearkes ${ }^{151}$, M. Pedersen ${ }^{131}$, L. Pedraza Diaz ${ }^{116}$,

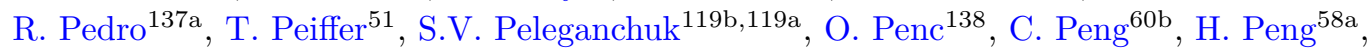
M. Penzin ${ }^{163}$, B.S. Peralva ${ }^{78 a}$, M.M. Perego ${ }^{62}$, A.P. Pereira Peixoto ${ }^{137 a}$, L. Pereira Sanchez ${ }^{43 a, 43 b}$, D.V. Perepelitsa ${ }^{27}$, E. Perez Codina ${ }^{165 a}$, M. Perganti ${ }^{9}$, L. Perini ${ }^{66 a}, 66 b$, H. Pernegger ${ }^{34}$, S. Perrella ${ }^{34}$, A. Perrevoort ${ }^{117}$, K. Peters ${ }^{44}$, R.F.Y. Peters ${ }^{98}$, B.A. Petersen ${ }^{34}$, T.C. Petersen ${ }^{38}$,

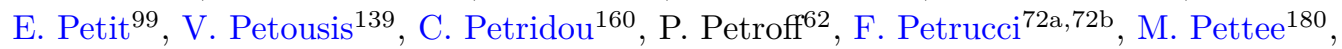
N.E. Pettersson ${ }^{100}$, K. Petukhova ${ }^{140}$, A. Peyaud ${ }^{142}$, R. Pezoa ${ }^{144}$, L. Pezzotti68a,68b, G. Pezzullo ${ }^{180}$, T. Pham ${ }^{102}$, P.W. Phillips ${ }^{141}$, M.W. Phipps ${ }^{170}$, G. Piacquadio ${ }^{153}$, E. Pianori ${ }^{16}$, F. Piazza ${ }^{66 a, 66 b}$, A. Picazio ${ }^{100}$, R. Piegaia ${ }^{28}$, D. Pietreanu ${ }^{25 b}$, J.E. Pilcher ${ }^{35}$, A.D. Pilkington ${ }^{98}$, M. Pinamonti ${ }^{64 a, 64 c}$, J.L. Pinfold ${ }^{2}$, C. Pitman Donaldson ${ }^{92}$, D.A. Pizzi ${ }^{32}$, L. Pizzimento ${ }^{71 a, 71 b}$, A. Pizzini ${ }^{117}$, M.-A. Pleier ${ }^{27}$, V. Plesanovs ${ }^{50}$, V. Pleskot ${ }^{140}$, E. Plotnikova ${ }^{77}$, P. Podberezko 119b,119a, R. Poettgen ${ }^{94}$, R. Poggi ${ }^{52}$, L. Poggioli ${ }^{133}$, I. Pogrebnyak ${ }^{104}$, D. Pohl ${ }^{22}$,

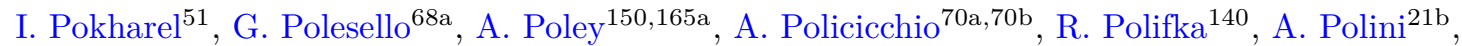
C.S. Pollard ${ }^{44}$, Z.B. Pollock ${ }^{125}$, V. Polychronakos ${ }^{27}$, D. Ponomarenko ${ }^{109}$, L. Pontecorvo ${ }^{34}$, S. Popa ${ }^{25 a}$, G.A. Popeneciu ${ }^{25 d}$, L. Portales ${ }^{4}$, D.M. Portillo Quintero ${ }^{56}$, S. Pospisil ${ }^{139}$, P. Postolache ${ }^{25 c}$, K. Potamianos ${ }^{132}$, I.N. Potrap ${ }^{77}$, C.J. Potter ${ }^{30}$, H. Potti ${ }^{10}$, T. Poulsen ${ }^{44}$, J. Poveda ${ }^{171}$, T.D. Powell ${ }^{147}$, G. Pownall ${ }^{44}$, M.E. Pozo Astigarraga ${ }^{34}$, A. Prades Ibanez ${ }^{171}$, P. Pralavorio ${ }^{99}$, M.M. Prapa ${ }^{42}$, S. Prell ${ }^{76}$, D. Price ${ }^{98}$, M. Primavera ${ }^{65 a}$, M.A. Principe Martin ${ }^{96}$, M.L. Proffitt ${ }^{146}$, N. Proklova ${ }^{109}$, K. Prokofiev ${ }^{60 c}$, F. Prokoshin ${ }^{77}$, S. Protopopescu ${ }^{27}$, J. Proudfoot ${ }^{5}$, M. Przybycien ${ }^{81 a}$, D. Pudzha ${ }^{135}$, P. Puzo ${ }^{62}$, D. Pyatiizbyantseva ${ }^{109}$, J. Qian ${ }^{103}$, Y. Qin ${ }^{98}$, A. Quadt ${ }^{51}$, M. Queitsch-Maitland ${ }^{34}$, G. Rabanal Bolanos ${ }^{57}$, F. Ragusa ${ }^{66 a, 66 b}$, G. Rahal ${ }^{95}$, J.A. Raine ${ }^{52}$, S. Rajagopalan ${ }^{27}$, K. Ran ${ }^{13 a, 13 d}$, D.F. Rassloff59a, D.M. Rauch ${ }^{44}$, S. Rave ${ }^{97}$, B. Ravina ${ }^{55}$, I. Ravinovich ${ }^{177}$, M. Raymond ${ }^{34}$, A.L. Read ${ }^{131}$, N.P. Readioff ${ }^{147}$, M. Reale ${ }^{65 a, 65 b}$, D.M. Rebuzzi $68 \mathrm{a}, 68 \mathrm{~b}$, G. Redlinger ${ }^{27}$, K. Reeves ${ }^{41}$, D. Reikher ${ }^{159}$, A. Reiss ${ }^{97}$, A. Rej ${ }^{149}$, C. Rembser ${ }^{34}$, A. Renardi ${ }^{44}$, M. Renda ${ }^{25 b}$, M.B. Rendel ${ }^{112}$, A.G. Rennie ${ }^{55}$, S. Resconi ${ }^{66 a}$, E.D. Resseguie ${ }^{16}$, S. Rettie ${ }^{92}$, B. Reynolds ${ }^{125}$, E. Reynolds ${ }^{19}$, M. Rezaei Estabragh ${ }^{179}$, O.L. Rezanova119b,119a, P. Reznicek ${ }^{140}$, E. Ricci ${ }^{73 a, 73 b}$, R. Richter ${ }^{112}$, S. Richter ${ }^{44}$, E. Richter-Was ${ }^{81 b}$, M. Ridel ${ }^{133}$, P. Rieck $^{112}$, P. Riedler ${ }^{34}$, O. Rifki ${ }^{44}$, M. Rijssenbeek ${ }^{153}$, A. Rimoldi ${ }^{68 a, 68 b}$, M. Rimoldi ${ }^{44}$, L. Rinaldi ${ }^{21 b, 21 a}$, T.T. Rinn ${ }^{170}$, M.P. Rinnagel ${ }^{111}$, G. Ripellino ${ }^{152}$, I. Riu ${ }^{12}$, P. Rivadeneira ${ }^{44}$, J.C. Rivera Vergara ${ }^{173}$, F. Rizatdinova ${ }^{127}$, E. Rizvi ${ }^{90}$, C. Rizzi ${ }^{52}$, S.H. Robertson ${ }^{101, \text { aa }}$, M. Robin ${ }^{44}$, D. Robinson ${ }^{30}$, C.M. Robles Gajardo ${ }^{144 f}$, M. Robles Manzano ${ }^{97}$, A. Robson ${ }^{55}$, A. Rocchi ${ }^{71 a, 71 b}$, C. Roda ${ }^{69 a, 69 b}$, S. Rodriguez Bosca ${ }^{59 a}$, A. Rodriguez Rodriguez ${ }^{50}$, A.M. Rodríguez Vera ${ }^{165 b}$, S. Roe ${ }^{34}$, J. Roggel ${ }^{179}$, O. Røhne ${ }^{131}$, R.A. Rojas ${ }^{144 f}$, B. Roland ${ }^{50}$, C.P.A. Roland ${ }^{63}$, J. Roloff ${ }^{27}$, A. Romaniouk ${ }^{109}$, M. Romano ${ }^{21 b}$, N. Rompotis ${ }^{88}$, M. Ronzani ${ }^{123}$, L. Roos ${ }^{133}$, S. Rosati ${ }^{70 a}$, G. $\operatorname{Rosin}^{100}$, B.J. Rosser ${ }^{134}$, E. Rossi ${ }^{164}$, E. Rossi ${ }^{4}$, E. Rossi ${ }^{67 a}$,67b , L.P. Rossi ${ }^{53 b}$, L. Rossini ${ }^{44}$, R. Rosten ${ }^{125}$, M. Rotaru ${ }^{25 b}$, B. Rottler ${ }^{50}$, D. Rousseau ${ }^{62}$, D. Rousso ${ }^{30}$, G. Rovelli68a,68b, A. $\operatorname{Roy}^{10}$, A. Rozanov ${ }^{99}$, Y. Rozen ${ }^{158}$, X. Ruan $^{31 f}$, A.J. Ruby ${ }^{88}$, T.A. Ruggeri ${ }^{1}$, F. Rühr ${ }^{50}$, A. Ruiz-Martinez ${ }^{171}$, A. Rummler ${ }^{34}$, Z. Rurikova ${ }^{50}$, N.A. Rusakovich ${ }^{77}$, H.L. Russell ${ }^{34}$, L. Rustige ${ }^{36}$, J.P. Rutherfoord ${ }^{6}$, E.M. Rüttinger ${ }^{147}$, M. Rybar ${ }^{140}$, E.B. Rye ${ }^{131}$, A. Ryzhov ${ }^{120}$, J.A. Sabater Iglesias ${ }^{44}$, P. Sabatini ${ }^{171}$, L. Sabetta ${ }^{70 a, 70 b}$, H.F-W. Sadrozinski ${ }^{143}$, R. Sadykov ${ }^{77}$, F. Safai Tehrani ${ }^{70 a}$, B. Safarzadeh Samani ${ }^{154}$, M. Safdari ${ }^{151}$, P. Saha ${ }^{118}$, S. Saha ${ }^{101}$, M. Sahinsoy ${ }^{112}$, A. Sahu ${ }^{179}$, M. Saimpert ${ }^{34}$, M. Saito ${ }^{161}$, T. Saito ${ }^{161}$, D. Salamani ${ }^{52}$, G. Salamanna ${ }^{72 a, 72 b}$, A. Salnikov ${ }^{151}$, J. Salt ${ }^{171}$, A. Salvador Salas ${ }^{12}$, D. Salvatore ${ }^{39 b, 39 a}$, F. Salvatore ${ }^{154}$, A. Salzburger ${ }^{34}$, D. Sammel ${ }^{50}$, D. Sampsonidis ${ }^{160}$, D. Sampsonidou ${ }^{58 d, 58 c}$, J. Sánchez ${ }^{171}$, A. Sanchez Pineda ${ }^{4}$, V. Sanchez Sebastian ${ }^{171}$, H. Sandaker ${ }^{131}$, C.O. Sander ${ }^{44}$, 
I.G. Sanderswood ${ }^{87}$, J.A. Sandesara ${ }^{100}$, M. Sandhoff ${ }^{179}$, C. Sandoval ${ }^{20 b}$, D.P.C. Sankey ${ }^{141}$, M. Sannino ${ }^{53 b, 53 a}$, Y. Sano ${ }^{114}$, A. Sansoni ${ }^{49}$, C. Santoni ${ }^{36}$, H. Santos ${ }^{137 a, 137 b}$, S.N. Santpur ${ }^{16}$, A. Santra ${ }^{177}$, K.A. Saoucha ${ }^{147}$, A. Sapronov ${ }^{77}$, J.G. Saraiva ${ }^{137 a, 137 d}$, J. Sardain ${ }^{99}$, O. Sasaki ${ }^{79}$, K. Sato ${ }^{166}$, C. Sauer ${ }^{59 b}$, F. Sauerburger ${ }^{50}$, E. Sauvan ${ }^{4}$, P. Savard ${ }^{164, a k}$, R. Sawada ${ }^{161}$, C. Sawyer ${ }^{141}$, L. Sawyer ${ }^{93}$, I. Sayago Galvan ${ }^{171}$, C. Sbarra ${ }^{21 b}$, A. Sbrizzi ${ }^{64 a, 64 c}$, T. Scanlon ${ }^{92}$, J. Schaarschmidt ${ }^{146}$, P. Schacht ${ }^{112}$, D. Schaefer ${ }^{35}$, L. Schaefer ${ }^{134}$, U. Schäfer ${ }^{97}$, A.C. Schaffer ${ }^{62}$, D. Schaile ${ }^{111}$, R.D. Schamberger ${ }^{153}$, E. Schanet ${ }^{111}$, C. Scharf $^{17}$, N. Scharmberg ${ }^{98}$, V.A. Schegelsky ${ }^{135}$, D. Scheirich ${ }^{140}$, F. Schenck ${ }^{17}$, M. Schernau ${ }^{168}$, C. Schiavi ${ }^{53 b, 53 a}$, L.K. Schildgen ${ }^{22}$, Z.M. Schillaci ${ }^{24}$,E.J. Schioppa ${ }^{65 a, 65 b}$, M. Schioppa ${ }^{39 b, 39 a}$, B. Schlag97, K.E. Schleicher ${ }^{50}$, S. Schlenker ${ }^{34}$, K. Schmieden ${ }^{97}$, C. Schmitt ${ }^{97}$, S. Schmitt ${ }^{44}$, L. Schoeffel ${ }^{142}$, A. Schoening ${ }^{59 b}$, P.G. Scholer ${ }^{50}$, E. Schopf ${ }^{132}$, M. Schott ${ }^{97}$, J. Schovancova ${ }^{34}$, S. Schramm ${ }^{52}$, F. Schroeder ${ }^{179}$, H-C. Schultz-Coulon ${ }^{59 a}$, M. Schumacher ${ }^{50}$, B.A. Schumm ${ }^{143}$, Ph. Schune ${ }^{142}$, A. Schwartzman ${ }^{151}$, T.A. Schwarz ${ }^{103}$, Ph. Schwemling ${ }^{142}$, R. Schwienhorst ${ }^{104}$, A. Sciandra ${ }^{143}$, G. Sciolla ${ }^{24}$, F. Scuri ${ }^{69 a}$, F. Scutti ${ }^{102}$, C.D. Sebastiani ${ }^{88}$, K. Sedlaczek ${ }^{45}$, P. Seema ${ }^{17}$, S.C. Seidel ${ }^{115}$, A. Seiden ${ }^{143}$, B.D. Seidlitz ${ }^{27}$, T. Seiss ${ }^{35}$, C. Seitz ${ }^{44}$, J.M. Seixas ${ }^{78 b}$, G. Sekhniaidze ${ }^{67 a}$, S.J. Sekula ${ }^{40}$, L.P. Selem ${ }^{4}$, N. Semprini-Cesari ${ }^{21 b, 21 a}$, S. Sen ${ }^{47}$, C. Serfon ${ }^{27}$, L. Serin ${ }^{62}$, L. Serkin $64 a, 64 b$, M. Sessa ${ }^{58 a}$, H. Severini ${ }^{126}$, S. Sevova ${ }^{151}$, F. Sforza ${ }^{53 b, 53 a}$, A. Sfyrla ${ }^{52}$, E. Shabalina ${ }^{51}$, J.D. Shahinian ${ }^{134}$, N.W. Shaikh43a,43b, D. Shaked Renous ${ }^{177}$, L.Y. Shan ${ }^{13 a}$, M. Shapiro ${ }^{16}$, A. Sharma ${ }^{34}$, A.S. Sharma ${ }^{1}$, S. Sharma ${ }^{44}$, P.B. Shatalov ${ }^{121}$, K. Shaw ${ }^{154}$, S.M. Shaw ${ }^{98}$, M. Shehade ${ }^{177}$, P. Sherwood ${ }^{92}$, L. Shi ${ }^{92}$, C.O. Shimmin ${ }^{180}$, Y. Shimogama ${ }^{176}$, M. Shimojima ${ }^{113}$, J.D. Shinner ${ }^{91}$, I.P.J. Shipsey ${ }^{132}$, S. Shirabe ${ }^{52}$, M. Shiyakova ${ }^{77}$, J. Shlomi ${ }^{177}$, M.J. Shochet ${ }^{35}$, J. Shojaii ${ }^{102}$, D.R. Shope ${ }^{152}$, S. Shrestha ${ }^{125}$, E.M. Shrif ${ }^{31 f}$, M.J. Shroff ${ }^{173}$, E. Shulga ${ }^{177}$, P. Sicho ${ }^{138}$, A.M. Sickles ${ }^{170}$, E. Sideras Haddad ${ }^{31 f}$, O. Sidiropoulou ${ }^{34}$, A. Sidoti ${ }^{21 b}$, F. Siegert ${ }^{46}$, Dj. Sijacki ${ }^{14}$, M.V. Silva Oliveira ${ }^{34}$, S.B. Silverstein ${ }^{43 a}$, S. Simion ${ }^{62}$, R. Simoniello ${ }^{34}$, S. Simsek ${ }^{11 b}$, P. Sinervo ${ }^{164}$, V. Sinetckii ${ }^{110}$, S. Singh ${ }^{150}$, S. Sinha ${ }^{44}$, S. Sinha ${ }^{31 f}$, M. Sioli ${ }^{21 b, 21 a}$, I. Siral ${ }^{129}$, S.Yu. Sivoklokov ${ }^{110}$, J. Sjölin ${ }^{43 a, 43 b}$, A. Skaf ${ }^{51}$, E. Skorda ${ }^{94}$, P. Skubic ${ }^{126}$,

M. Slawinska ${ }^{82}$, K. Sliwa ${ }^{167}$, V. Smakhtin ${ }^{177}$, B.H. Smart ${ }^{141}$, J. Smiesko ${ }^{140}$, S.Yu. Smirnov ${ }^{109}$, Y. Smirnov ${ }^{109}$, L.N. Smirnova ${ }^{110, s}$, O. Smirnova ${ }^{94}$, E.A. Smith ${ }^{35}$, H.A. Smith ${ }^{132}$, M. Smizanska ${ }^{87}$, K. Smolek ${ }^{139}$, A. Smykiewicz ${ }^{82}$, A.A. Snesarev ${ }^{108}$, H.L. Snoek ${ }^{117}$, I.M. Snyder ${ }^{129}$, S. Snyder ${ }^{27}$, R. Sobie ${ }^{173, \text { aa }}$, A. Soffer ${ }^{159}$, A. Søgaard ${ }^{48}$, F. Sohns ${ }^{51}$, C.A. Solans Sanchez ${ }^{34}$, E.Yu. Soldatov ${ }^{109}$, U. Soldevila ${ }^{171}$, A.A. Solodkov ${ }^{120}$, S. Solomon ${ }^{50}$, A. Soloshenko ${ }^{77}$, O.V. Solovyanov ${ }^{120}$, V. Solovyev ${ }^{135}$, P. Sommer ${ }^{147}$, H. Son ${ }^{167}$, A. Sonay ${ }^{12}$, W.Y. Song ${ }^{165 b}$, A. Sopczak ${ }^{139}$, A.L. Sopio ${ }^{92}$, F. Sopkova ${ }^{26 b}$, S. Sottocornola ${ }^{68 a, 68 b}$, R. Soualah ${ }^{64 a, 64 c}$, A.M. Soukharev ${ }^{119 b, 119 a,}$ Z. Soumaimi ${ }^{33 e}$, D. South ${ }^{44}$, S. Spagnolo ${ }^{65 a, 65 b}$, M. Spalla ${ }^{112}$, M. Spangenberg ${ }^{175}$, F. Spanò ${ }^{91}$, D. Sperlich ${ }^{50}$, T.M. Spieker ${ }^{59 a}$, G. Spigo $^{34}$, M. Spina ${ }^{154}$, D.P. Spiteri ${ }^{55}$, M. Spousta ${ }^{140}$, A. Stabile $66 a, 66 b$, B.L. Stamas ${ }^{118}$, R. Stamen ${ }^{59 a}$, M. Stamenkovic ${ }^{117}$, A. Stampekis ${ }^{19}$, M. Standke ${ }^{22}$, E. Stanecka ${ }^{82}$, B. Stanislaus ${ }^{34}$, M.M. Stanitzki ${ }^{44}$, M. Stankaityte ${ }^{132}$, B. Stapf ${ }^{44}$, E.A. Starchenko ${ }^{120}$, G.H. Stark ${ }^{143}$, J. Stark ${ }^{99}$, D.M. Starko ${ }^{165 b}$, P. Staroba ${ }^{138}$, P. Starovoitov ${ }^{59 a}$, S. Stärz ${ }^{101}$, R. Staszewski ${ }^{82}$, G. Stavropoulos ${ }^{42}$, P. Steinberg ${ }^{27}$, A.L. Steinhebel ${ }^{129}$, B. Stelzer ${ }^{150,165 a}$, H.J. Stelzer ${ }^{136}$, O. Stelzer-Chilton ${ }^{165 a}$, H. Stenzel ${ }^{54}$, T.J. Stevenson ${ }^{154}$, G.A. Stewart ${ }^{34}$, M.C. Stockton ${ }^{34}$, G. Stoicea ${ }^{25 b}$, M. Stolarski ${ }^{137 a}$, S. Stonjek ${ }^{112}$, A. Straessner ${ }^{46}$, J. Strandberg ${ }^{152}$, S. Strandberg ${ }^{43 a, 43 b}$, M. Strauss ${ }^{126}$, T. Strebler ${ }^{99}$, P. Strizenec ${ }^{26 b}$, R. Ströhmer ${ }^{174}$, D.M. Strom ${ }^{129}$, L.R. Strom $^{44}$, R. Stroynowski ${ }^{40}$, A. Strubig ${ }^{43 a, 43 b}$, S.A. Stucci ${ }^{27}$, B. Stugu ${ }^{15}$, J. Stupak ${ }^{126}$, N.A. Styles ${ }^{44}$, D. Su${ }^{151}$, S. Su ${ }^{58 a}$, W. Su ${ }^{58 d, 146,58 c}$, X. Su ${ }^{58 a}$, N.B. Suarez ${ }^{136}$, K. Sugizaki ${ }^{161}$, V.V. Sulin ${ }^{108}$, M.J. Sullivan ${ }^{88}$, D.M.S. Sultan ${ }^{52}$, S. Sultansoy ${ }^{3 c}$, T. Sumida ${ }^{83}$, S. Sun ${ }^{103}$, S. Sun ${ }^{178}$, X. Sun ${ }^{98}$, O. Sunneborn Gudnadottir ${ }^{169}$, C.J.E. Suster ${ }^{155}$, M.R. Sutton ${ }^{154}$, M. Svatos ${ }^{138}$, M. Swiatlowski ${ }^{165 a}$, T. Swirski ${ }^{174}$, I. Sykora ${ }^{26 a}$, M. Sykora ${ }^{140}$,

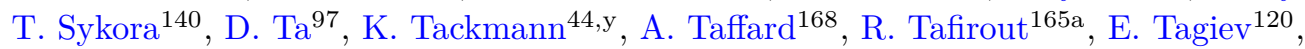


R.H.M. Taibah ${ }^{133}$, R. Takashima ${ }^{84}$, K. Takeda ${ }^{80}$, T. Takeshita ${ }^{148}$, E.P. Takeva ${ }^{48}$, Y. Takubo ${ }^{79}$, M. Talby ${ }^{99}$, A.A. Talyshev ${ }^{119 b, 119 a}$, K.C. Tam ${ }^{60 b}$, N.M. Tamir ${ }^{159}$, J. Tanaka ${ }^{161}$, R. Tanaka ${ }^{62}$, Z. Tao ${ }^{172}$, S. Tapia Araya ${ }^{170}$, S. Tapprogge ${ }^{97}$, A. Tarek Abouelfadl Mohamed ${ }^{104}$, S. Tarem ${ }^{158}$,

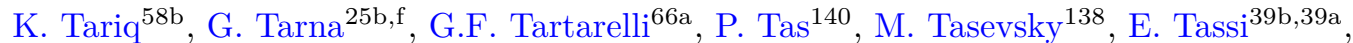
G. Tateno ${ }^{161}$, Y. Tayalati ${ }^{33 e}$, G.N. Taylor ${ }^{102}$, W. Taylor ${ }^{165 b}$, H. Teagle ${ }^{88}$, A.S. Tee ${ }^{87}$, R. Teixeira De Lima ${ }^{151}$, P. Teixeira-Dias ${ }^{91}$, H. Ten Kate ${ }^{34}$, J.J. Teoh ${ }^{117}$, K. Terashi ${ }^{161}$, J. Terron ${ }^{96}$, S. Terzo ${ }^{12}$, M. Testa ${ }^{49}$, R.J. Teuscher ${ }^{164, \text { aa }}$, N. Themistokleous ${ }^{48}$,

T. Theveneaux-Pelzer ${ }^{17}$, D.W. Thomas ${ }^{91}$, J.P. Thomas ${ }^{19}$, E.A. Thompson ${ }^{44}$, P.D. Thompson ${ }^{19}$, E. Thomson ${ }^{134}$, E.J. Thorpe ${ }^{90}$, Y. Tian ${ }^{51}$, V.O. Tikhomirov ${ }^{108, a h}$, Yu.A. Tikhonov ${ }^{119 b, 119 a}$,

S. Timoshenko ${ }^{109}$, P. Tipton ${ }^{180}$, S. Tisserant ${ }^{99}$, S.H. Tlou ${ }^{31 f}$, A. Tnourji ${ }^{36}$, K. Todome ${ }^{21 b, 21 a}$, S. Todorova-Nova ${ }^{140}$, S. Todt ${ }^{46}$, M. Togawa ${ }^{79}$, J. Tojo ${ }^{85}$, S. Tokár ${ }^{26 a}$, K. Tokushuku ${ }^{79}$, E. Tolley ${ }^{125}$, R. Tombs ${ }^{30}$, M. Tomoto ${ }^{79,114}$, L. Tompkins ${ }^{151}$, P. Tornambe ${ }^{100}$, E. Torrence ${ }^{129}$, H. Torres ${ }^{46}$, E. Torró Pastor ${ }^{171}$, M. Toscani ${ }^{28}$, C. Tosciri ${ }^{35}$, J. Toth ${ }^{99, z}$, D.R. Tovey ${ }^{147}$,

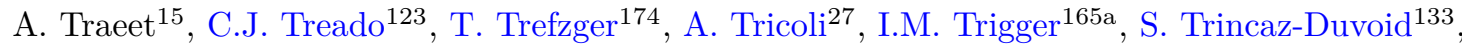

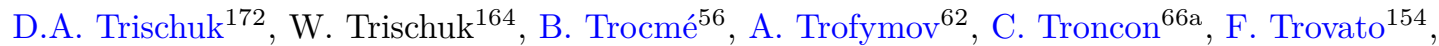
L. Truong ${ }^{31 c}$, M. Trzebinski ${ }^{82}$, A. Trzupek ${ }^{82}$, F. Tsai ${ }^{153}$, A. Tsiamis ${ }^{160}$, P.V. Tsiareshka ${ }^{105, a f}$, A. Tsirigotis ${ }^{160, w}$, V. Tsiskaridze ${ }^{153}$, E.G. Tskhadadze ${ }^{157 a}$, M. Tsopoulou ${ }^{160}$, I.I. Tsukerman ${ }^{121}$,

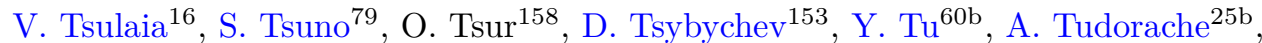
V. Tudorache ${ }^{25 b}$, A.N. Tuna ${ }^{34}$, S. Turchikhin ${ }^{77}$, D. Turgeman ${ }^{177}$, I. Turk Cakir ${ }^{3 b, u}$, R.J. Turner ${ }^{19}$, R. Turra ${ }^{66 a}$, P.M. Tuts ${ }^{37}$, S. Tzamarias ${ }^{160}$, P. Tzanis ${ }^{9}$, E. Tzovara ${ }^{97}$, K. Uchida ${ }^{161}$, F. Ukegawa ${ }^{166}$,

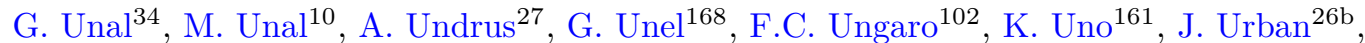
P. Urquijo ${ }^{102}$, G. Usai ${ }^{7}$, R. Ushioda ${ }^{162}$, Z. Uysal ${ }^{11 d}$, V. Vacek ${ }^{139}$, B. Vachon ${ }^{101}$, K.O.H. Vadla ${ }^{131}$ T. Vafeiadis ${ }^{34}$, C. Valderanis ${ }^{111}$, E. Valdes Santurio ${ }^{43 a, 43 b}$, M. Valente ${ }^{165 a}$, S. Valentinetti ${ }^{21 b, 21 a}$, A. Valero ${ }^{171}$, L. Valéry ${ }^{44}$, R.A. Vallance ${ }^{19}$, A. Vallier ${ }^{99}$, J.A. Valls Ferrer ${ }^{171}$, T.R. Van Daalen ${ }^{12}$, P. Van Gemmeren ${ }^{5}$, S. Van Stroud ${ }^{92}$, I. Van Vulpen ${ }^{117}$, M. Vanadia ${ }^{71 a, 71 b}$, W. Vandelli ${ }^{34}$, M. Vandenbroucke ${ }^{142}$, E.R. Vandewall ${ }^{127}$, D. Vannicola ${ }^{70 a, 70 b}$, L. Vannoli $^{53 b, 53 a}$, R. Vari ${ }^{70 a}$, E.W. Varnes ${ }^{6}$, C. Varni ${ }^{53 b, 53 a}$, T. $V^{2}{ }^{150}{ }^{156}$, D. $\operatorname{Varouchas}^{62}$, K.E. Varvell ${ }^{155}$, M.E. Vasile ${ }^{25 b}$, L. Vaslin ${ }^{36}$, G.A. Vasquez ${ }^{173}$, F. Vazeille ${ }^{36}$, D. Vazquez Furelos ${ }^{12}$, T. Vazquez Schroeder ${ }^{34}$, J. Veatch ${ }^{51}$, V. Vecchio ${ }^{98}$, M.J. Veen ${ }^{117}$, I. Veliscek ${ }^{132}$, L.M. Veloce ${ }^{164}$, F. Veloso ${ }^{137 a, 137 c}$,

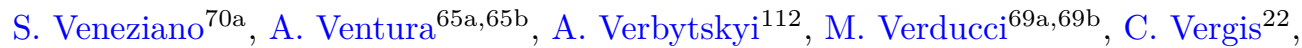
M. Verissimo De Araujo ${ }^{78 b}$, W. Verkerke ${ }^{117}$, A.T. Vermeulen ${ }^{117}$, J.C. Vermeulen ${ }^{117}$,

C. Vernieri ${ }^{151}$, P.J. Verschuuren ${ }^{91}$, M.L. Vesterbacka ${ }^{123}$, M.C. Vetterli150,ak, N. Viaux Maira ${ }^{144 f}$, T. Vickey ${ }^{147}$, O.E. Vickey Boeriu ${ }^{147}$, G.H.A. Viehhauser ${ }^{132}$, L. Vigani ${ }^{59 b}$, M. Villa ${ }^{21 b, 21 a}$, M. Villaplana Perez ${ }^{171}$, E.M. Villhauer ${ }^{48}$, E. Vilucchi ${ }^{49}$, M.G. Vincter ${ }^{32}$, G.S. Virdee ${ }^{19}$, A. Vishwakarma ${ }^{48}$, C. Vittori ${ }^{21 b, 21 a}$, I. Vivarelli ${ }^{154}$, V. Vladimirov ${ }^{175}$, E. Voevodina ${ }^{112}$, M. Vogel ${ }^{179}$, P. Vokac ${ }^{139}$, J. Von Ahnen ${ }^{44}$, S.E. von Buddenbrock ${ }^{31 f}$, E. Von Toerne ${ }^{22}$,

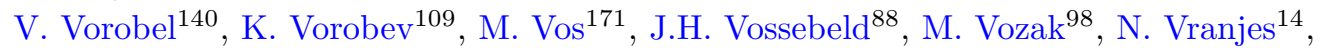
M. Vranjes Milosavljevic ${ }^{14}$, V. Vrba ${ }^{139, *}$, M. Vreeswijk ${ }^{117}$, N.K. Vu ${ }^{99}$, R. Vuillermet ${ }^{34}$, I. Vukotic ${ }^{35}$, S. Wada ${ }^{166}$, C. Wagner ${ }^{100}$, P. Wagner ${ }^{22}$, W. Wagner ${ }^{179}$, S. Wahdan ${ }^{179}$, H. Wahlberg ${ }^{86}$, R. Wakasa ${ }^{166}$, M. Wakida ${ }^{114}$, V.M. Walbrecht ${ }^{112}$, J. Walder ${ }^{141}$, R. Walker ${ }^{111}$, S.D. Walker ${ }^{91}$, W. Walkowiak ${ }^{149}$, A.M. Wang ${ }^{57}$, A.Z. Wang ${ }^{178}$, C. Wang ${ }^{58 a}$, C. Wang ${ }^{58 c}$, H. Wang ${ }^{16}$, J. Wang ${ }^{60 a}$, P. Wang ${ }^{40}$, R.-J. Wang ${ }^{97}$, R. Wang ${ }^{57}$, R. Wang ${ }^{118}$, S.M. Wang ${ }^{156}$,

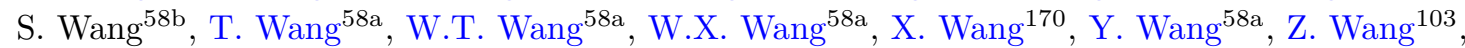

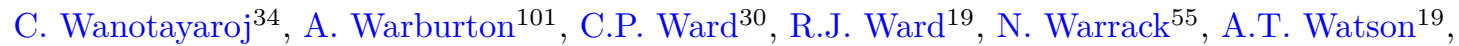
M.F. Watson ${ }^{19}$, G. Watts ${ }^{146}$, B.M. Waugh ${ }^{92}$, A.F. Webb ${ }^{10}$, C. Weber ${ }^{27}$, M.S. Weber ${ }^{18}$, S.A. Weber ${ }^{32}$, S.M. Weber ${ }^{59 a}$, C. Wei ${ }^{58 a}$, Y. Wei ${ }^{132}$, A.R. Weidberg ${ }^{132}$, J. Weingarten ${ }^{45}$,

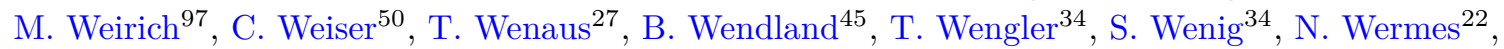
M. Wessels ${ }^{59 a}$, K. Whalen ${ }^{129}$, A.M. Wharton ${ }^{87}$, A.S. White ${ }^{57}$, A. White ${ }^{7}$, M.J. White ${ }^{1}$, 


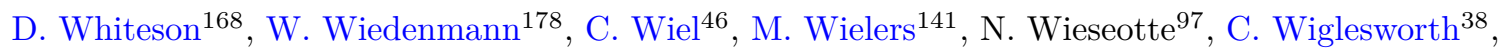
L.A.M. Wiik-Fuchs ${ }^{50}$, D.J. Wilbern ${ }^{126}$, H.G. Wilkens ${ }^{34}$, L.J. Wilkins ${ }^{91}$, D.M. Williams ${ }^{37}$, H.H. Williams ${ }^{134}$, S. Williams ${ }^{30}$, S. Willocq ${ }^{100}$, P.J. Windischhofer ${ }^{132}$, I. Wingerter-Seez ${ }^{4}$,

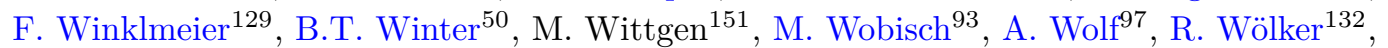
J. Wollrath ${ }^{168}$, M.W. Wolter ${ }^{82}$, H. Wolters ${ }^{137 a, 137 c}$, V.W.S. Wong ${ }^{172}$, A.F. Wongel ${ }^{44}$, S.D. Worm ${ }^{44}$, B.K. Wosiek ${ }^{82}$, K.W. Woźniak ${ }^{82}$, K. Wraight ${ }^{55}$, J. Wu ${ }^{13 a, 13 d}$, S.L. Wu ${ }^{178}$, X. Wu ${ }^{52}$, Y. Wu ${ }^{58 \mathrm{a}}, \mathrm{Z} . \mathrm{Wu}^{142,58 \mathrm{a}}$, J. Wuerzinger ${ }^{132}$, T.R. Wyatt ${ }^{98}$, B.M. Wynne ${ }^{48}$, S. Xella ${ }^{38}$, J. Xiang $^{60 \mathrm{c}}$, X. Xiao ${ }^{103}, X . X^{58 a}$, I. Xiotidis ${ }^{154}$, D. Xu ${ }^{13 a}$, H. Xu ${ }^{58 a}$, H. Xu ${ }^{58 a}$, L. Xu ${ }^{58 a}$, R. Xu ${ }^{134}$, W. Xu ${ }^{103}$, Y. Xu ${ }^{13 b}$, Z. Xu ${ }^{58 b}$, Z. Xu ${ }^{151}$, B. Yabsley ${ }^{155}$, S. Yacoob ${ }^{31 a}$, N. Yamaguchi ${ }^{85}$, Y. Yamaguchi ${ }^{162}$, M. Yamatani ${ }^{161}$, H. Yamauchi ${ }^{166}$, T. Yamazaki ${ }^{16}$, Y. Yamazaki ${ }^{80}$, J. Yan $^{58 c}$, Z. Yan ${ }^{23}$,

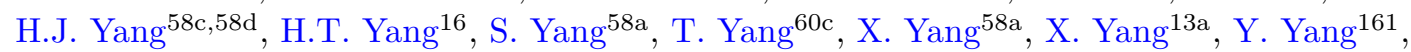
Z. Yang ${ }^{103,58 a}$, W-M. Yao ${ }^{16}$, Y.C. Yap ${ }^{44}$, H. Ye ${ }^{13 c}$, J. Ye ${ }^{40}$, S. Ye ${ }^{27}$, I. Yeletskikh ${ }^{77}$, M.R. Yexley ${ }^{87}$, P. Yin ${ }^{37}$, K. Yorita ${ }^{176}$, K. Yoshihara ${ }^{76}$, C.J.S. Young ${ }^{34}$, C. Young ${ }^{151}$, R. Yuan ${ }^{58 b, j}$, X. Yue ${ }^{59 a}$, M. Zaazoua ${ }^{33 e}$, B. Zabinski ${ }^{82}$, G. Zacharis ${ }^{9}$, E. Zaffaroni ${ }^{52}$, A.M. Zaitsev ${ }^{120, a g}$, T. Zakareishvili ${ }^{157 b}$, N. Zakharchuk ${ }^{32}$, S. Zambito ${ }^{34}$, D. Zanzi ${ }^{50}$, S.V. Zeißner ${ }^{45}$, C. Zeitnitz ${ }^{179}$, G. Zemaityte ${ }^{132}$, J.C. Zeng ${ }^{170}$, O. Zenin ${ }^{120}$, T. Ženiš ${ }^{26 a}$, S. Zenz ${ }^{90}$, S. Zerradi ${ }^{33 a}$, D. Zerwas ${ }^{62}$, M. Zgubič ${ }^{132}$, B. Zhang ${ }^{13 c}$, D.F. Zhang ${ }^{13 b}$, G. Zhang ${ }^{13 b}$, J. Zhang ${ }^{5}$, K. Zhang ${ }^{13 a}$, L. Zhang ${ }^{13 c}$, M. Zhang ${ }^{170}$, R. Zhang ${ }^{178}$, S. Zhang ${ }^{103}$, X. Zhang ${ }^{58 c}$, X. Zhang ${ }^{58 b}$, Z. Zhang ${ }^{62}$, P. Zhao ${ }^{47}$, Y. Zhao ${ }^{143}$, Z. Zhao ${ }^{58 a}$, A. Zhemchugov ${ }^{77}$, Z. Zheng ${ }^{103}$, D. Zhong ${ }^{170}$, B. Zhou ${ }^{103}$, C. Zhou ${ }^{178}$, H. Zhou ${ }^{6}$, M. Zhou ${ }^{153}$, N. Zhou ${ }^{58 c}$, Y. Zhou ${ }^{6}$, C.G. Zhu ${ }^{58 b}$, C. Zhu ${ }^{13 a, 13 d}$, H.L. Zhu ${ }^{58 a}$, H. Zhu ${ }^{13 a}$, J. Zhu ${ }^{103}$, Y. Zhu ${ }^{58 a}$, X. Zhuang13a , K. Zhukov ${ }^{108}$, V. Zhulanov ${ }^{119 b, 119 a}$, D. Zieminska ${ }^{63}$, N.I. Zimine ${ }^{77}$, S. Zimmermann ${ }^{50, *}$, M. Ziolkowski ${ }^{149}$, L. Živković ${ }^{14}$, A. Zoccoli ${ }^{21 b, 21 a}$, K. Zoch $^{52}$, T.G. Zorbas ${ }^{147}$, W. Zou ${ }^{37}$, L. Zwalinski ${ }^{34}$

1 Department of Physics, University of Adelaide, Adelaide; Australia

2 Department of Physics, University of Alberta, Edmonton AB; Canada

3 Department of Physics ${ }^{(a)}$, Ankara University, Ankara; Istanbul Aydin University ${ }^{(b)}$, Application and Research Center for Advanced Studies, Istanbul; Division of Physics ${ }^{(c)}$, TOBB University of Economics and Technology, Ankara; Turkey

4 LAPP, Univ. Savoie Mont Blanc, CNRS/IN2P3, Annecy; France

5 High Energy Physics Division, Argonne National Laboratory, Argonne IL; United States of America

6 Department of Physics, University of Arizona, Tucson AZ; United States of America

7 Department of Physics, University of Texas at Arlington, Arlington TX; United States of America

8 Physics Department, National and Kapodistrian University of Athens, Athens; Greece

9 Physics Department, National Technical University of Athens, Zografou; Greece

10 Department of Physics, University of Texas at Austin, Austin TX; United States of America

11 Bahcesehir University ${ }^{(a)}$, Faculty of Engineering and Natural Sciences, Istanbul; Istanbul Bilgi University $^{(b)}$, Faculty of Engineering and Natural Sciences, Istanbul; Department of Physics ${ }^{(c)}$, Bogazici University, Istanbul; Department of Physics Engineering ${ }^{(d)}$, Gaziantep University, Gaziantep; Department of Physics ${ }^{(e)}$, Istanbul University, Istanbul; Istinye University ${ }^{(f)}$, Sariyer, Istanbul; Turkey

12 Institut de Física d'Altes Energies (IFAE), Barcelona Institute of Science and Technology, Barcelona; Spain

13 Institute of High Energy Physics ${ }^{(a)}$, Chinese Academy of Sciences, Beijing; Physics Department ${ }^{(b)}$, Tsinghua University, Beijing; Department of Physics ${ }^{(c)}$, Nanjing University, Nanjing; University of Chinese Academy of Science $(U C A S)^{(d)}$, Beijing; China

14 Institute of Physics, University of Belgrade, Belgrade; Serbia

15 Department for Physics and Technology, University of Bergen, Bergen; Norway

16 Physics Division, Lawrence Berkeley National Laboratory and University of California, Berkeley CA; United States of America

17 Institut für Physik, Humboldt Universität zu Berlin, Berlin; Germany 
Albert Einstein Center for Fundamental Physics and Laboratory for High Energy Physics, University of Bern, Bern; Switzerland

19 School of Physics and Astronomy, University of Birmingham, Birmingham; United Kingdom

20 Facultad de Ciencias y Centro de Investigaciónes ${ }^{(a)}$, Universidad Antonio Nariño, Bogotá; Departamento de Física ${ }^{(b)}$, Universidad Nacional de Colombia, Bogotá; Colombia

21 Dipartimento di Fisica e Astronomia A. Righi ${ }^{(a)}$, Università di Bologna, Bologna; INFN Sezione di Bologna $^{(b)}$; Italy

22 Physikalisches Institut, Universität Bonn, Bonn; Germany

23 Department of Physics, Boston University, Boston MA; United States of America

24 Department of Physics, Brandeis University, Waltham MA; United States of America

25 Transilvania University of Brasov ${ }^{(a)}$, Brasov; Horia Hulubei National Institute of Physics and Nuclear Engineering ${ }^{(b)}$, Bucharest; Department of Physics ${ }^{(c)}$, Alexandru Ioan Cuza University of Iasi, Iasi; National Institute for Research and Development of Isotopic and Molecular Technologies $^{(d)}$, Physics Department, Cluj-Napoca; University Politehnica Bucharest ${ }^{(e)}$, Bucharest; West University in Timisoara ${ }^{(f)}$, Timisoara; Romania

26 Faculty of Mathematics ${ }^{(a)}$, Physics and Informatics, Comenius University, Bratislava; Department of Subnuclear Physics ${ }^{(b)}$, Institute of Experimental Physics of the Slovak Academy of Sciences, Kosice; Slovak Republic

27 Physics Department, Brookhaven National Laboratory, Upton NY; United States of America

28 Departamento de Física (FCEN) and IFIBA, Universidad de Buenos Aires and CONICET, Buenos Aires; Argentina

29 California State University, CA; United States of America

30 Cavendish Laboratory, University of Cambridge, Cambridge; United Kingdom

31 Department of Physics ${ }^{(a)}$, University of Cape Town, Cape Town; iThemba Labs ${ }^{(b)}$, Western Cape; Department of Mechanical Engineering Science ${ }^{(c)}$, University of Johannesburg, Johannesburg; National Institute of Physics ${ }^{(d)}$, University of the Philippines Diliman (Philippines); University of South Africa ${ }^{(e)}$, Department of Physics, Pretoria; School of Physics ${ }^{(f)}$, University of the Witwatersrand, Johannesburg; South Africa

32 Department of Physics, Carleton University, Ottawa ON; Canada

33 Faculté des Sciences Ain Chock ${ }^{(a)}$, Réseau Universitaire de Physique des Hautes Energies Université Hassan II, Casablanca; Faculté des Sciences ${ }^{(b)}$, Université Ibn-Tofail, Kénitra; Faculté des Sciences Semlalia ${ }^{(c)}$, Université Cadi Ayyad, LPHEA-Marrakech; LPMR ${ }^{(d)}$, Faculté des Sciences, Université Mohamed Premier, Oujda; Faculté des sciences ${ }^{(e)}$, Université Mohammed V, Rabat; Mohammed VI Polytechnic University ${ }^{(f)}$, Ben Guerir; Morocco

34 CERN, Geneva; Switzerland

35 Enrico Fermi Institute, University of Chicago, Chicago IL; United States of America

36 LPC, Université Clermont Auvergne, CNRS/IN2P3, Clermont-Ferrand; France

37 Nevis Laboratory, Columbia University, Irvington NY; United States of America

38 Niels Bohr Institute, University of Copenhagen, Copenhagen; Denmark

39 Dipartimento di Fisica ${ }^{(a)}$, Università della Calabria, Rende; INFN Gruppo Collegato di Cosenza ${ }^{(b)}$, Laboratori Nazionali di Frascati; Italy

40 Physics Department, Southern Methodist University, Dallas TX; United States of America

41 Physics Department, University of Texas at Dallas, Richardson TX; United States of America

42 National Centre for Scientific Research "Demokritos", Agia Paraskevi; Greece

43 Department of Physics ${ }^{(a)}$, Stockholm University; Oskar Klein Centre ${ }^{(b)}$, Stockholm; Sweden

44 Deutsches Elektronen-Synchrotron DESY, Hamburg and Zeuthen; Germany

45 Lehrstuhl für Experimentelle Physik IV, Technische Universität Dortmund, Dortmund; Germany

46 Institut für Kern- und Teilchenphysik, Technische Universität Dresden, Dresden; Germany

47 Department of Physics, Duke University, Durham NC; United States of America

48 SUPA - School of Physics and Astronomy, University of Edinburgh, Edinburgh; United Kingdom

49 INFN e Laboratori Nazionali di Frascati, Frascati; Italy

50 Physikalisches Institut, Albert-Ludwigs-Universität Freiburg, Freiburg; Germany 
Département de Physique Nucléaire et Corpusculaire, Université de Genève, Genève; Switzerland

${ }^{53}$ Dipartimento di Fisica ${ }^{(a)}$, Università di Genova, Genova; INFN Sezione di Genova ${ }^{(b)}$; Italy

${ }^{54}$ II. Physikalisches Institut, Justus-Liebig-Universität Giessen, Giessen; Germany

${ }_{55}$ SUPA - School of Physics and Astronomy, University of Glasgow, Glasgow; United Kingdom

${ }^{56}$ LPSC, Université Grenoble Alpes, CNRS/IN2P3, Grenoble INP, Grenoble; France

57 Laboratory for Particle Physics and Cosmology, Harvard University, Cambridge MA; United States of America

58 Department of Modern Physics and State Key Laboratory of Particle Detection and Electronicss ${ }^{(a)}$, University of Science and Technology of China, Hefei; Institute of Frontier and Interdisciplinary Science and Key Laboratory of Particle Physics and Particle Irradiation $(M O E)^{(b)}$, Shandong University, Qingdao; School of Physics and Astronomy ${ }^{(c)}$, Shanghai Jiao Tong University, Key Laboratory for Particle Astrophysics and Cosmology (MOE), SKLPPC, Shanghai; Tsung-Dao Lee Institute $^{(d)}$, Shanghai; China

59 Kirchhoff-Institut für Physik ${ }^{(a)}$, Ruprecht-Karls-Universität Heidelberg, Heidelberg; Physikalisches Institut $^{(b)}$, Ruprecht-Karls-Universität Heidelberg, Heidelberg; Germany

${ }^{60}$ Department of Physics ${ }^{(a)}$, Chinese University of Hong Kong, Shatin, N.T., Hong Kong; Department of Physics ${ }^{(b)}$, University of Hong Kong, Hong Kong; Department of Physics and Institute for Advanced Study ${ }^{(c)}$, Hong Kong University of Science and Technology, Clear Water Bay, Kowloon, Hong Kong; China

${ }^{61}$ Department of Physics, National Tsing Hua University, Hsinchu; Taiwan

${ }^{62}$ IJCLab, Université Paris-Saclay, CNRS/IN2P3, 91405, Orsay; France

63 Department of Physics, Indiana University, Bloomington IN; United States of America

${ }^{64}$ INFN Gruppo Collegato di Udine ${ }^{(a)}$, Sezione di Trieste, Udine; ICTP ${ }^{(b)}$, Trieste; Dipartimento Politecnico di Ingegneria e Architettura ${ }^{(c)}$, Università di Udine, Udine; Italy

${ }^{65}$ INFN Sezione di Lecce ${ }^{(a)}$; Dipartimento di Matematica e Fisica ${ }^{(b)}$, Università del Salento, Lecce; Italy

${ }^{66}$ INFN Sezione di Milano ${ }^{(a)}$; Dipartimento di Fisica ${ }^{(b)}$, Università di Milano, Milano; Italy

${ }^{67}$ INFN Sezione di Napoli ${ }^{(a)}$; Dipartimento di Fisica ${ }^{(b)}$, Università di Napoli, Napoli; Italy

68 INFN Sezione di Pavia( ${ }^{(a)}$; Dipartimento di Fisica ${ }^{(b)}$, Università di Pavia, Pavia; Italy

${ }^{69}$ INFN Sezione di Pisa ${ }^{(a)}$; Dipartimento di Fisica E. Fermi ${ }^{(b)}$, Università di Pisa, Pisa; Italy

70 INFN Sezione di Roma ${ }^{(a)}$; Dipartimento di Fisica ${ }^{(b)}$, Sapienza Università di Roma, Roma; Italy

${ }^{71}$ INFN Sezione di Roma Tor Vergata ${ }^{(a)}$; Dipartimento di Fisica ${ }^{(b)}$, Università di Roma Tor Vergata, Roma; Italy

72 INFN Sezione di Roma Tre ${ }^{(a)}$; Dipartimento di Matematica e Fisica ${ }^{(b)}$, Università Roma Tre, Roma; Italy

${ }^{73}$ INFN-TIFPA $^{(a)}$; Università degli Studi di Trento ${ }^{(b)}$, Trento; Italy

${ }^{74}$ Institut für Astro- und Teilchenphysik, Leopold-Franzens-Universität, Innsbruck; Austria

75 University of Iowa, Iowa City IA; United States of America

${ }^{76}$ Department of Physics and Astronomy, Iowa State University, Ames IA; United States of America

77 Joint Institute for Nuclear Research, Dubna; Russia

78 Departamento de Engenharia Elétrica ${ }^{(a)}$, Universidade Federal de Juiz de Fora (UFJF), Juiz de Fora; Universidade Federal do Rio De Janeiro COPPE/EE/IF ${ }^{(b)}$, Rio de Janeiro; Instituto de Física $^{(c)}$, Universidade de São Paulo, São Paulo; Brazil

79 KEK, High Energy Accelerator Research Organization, Tsukuba; Japan

80 Graduate School of Science, Kobe University, Kobe; Japan

81 AGH University of Science and Technology ${ }^{(a)}$, Faculty of Physics and Applied Computer Science, Krakow; Marian Smoluchowski Institute of Physics ${ }^{(b)}$, Jagiellonian University, Krakow; Poland

82 Institute of Nuclear Physics Polish Academy of Sciences, Krakow; Poland

83 Faculty of Science, Kyoto University, Kyoto; Japan

${ }^{84}$ Kyoto University of Education, Kyoto; Japan

85 Research Center for Advanced Particle Physics and Department of Physics, Kyushu University, Fukuoka; Japan 
Instituto de Física La Plata, Universidad Nacional de La Plata and CONICET, La Plata; Argentina Physics Department, Lancaster University, Lancaster; United Kingdom

88 Oliver Lodge Laboratory, University of Liverpool, Liverpool; United Kingdom

89 Department of Experimental Particle Physics, Jožef Stefan Institute and Department of Physics, University of Ljubljana, Ljubljana; Slovenia

90 School of Physics and Astronomy, Queen Mary University of London, London; United Kingdom

91 Department of Physics, Royal Holloway University of London, Egham; United Kingdom

92 Department of Physics and Astronomy, University College London, London; United Kingdom

93 Louisiana Tech University, Ruston LA; United States of America

94 Fysiska institutionen, Lunds universitet, Lund; Sweden

95 Centre de Calcul de l'Institut National de Physique Nucléaire et de Physique des Particules (IN2P3), Villeurbanne; France

96 Departamento de Física Teorica C-15 and CIAFF, Universidad Autónoma de Madrid, Madrid; Spain

97 Institut für Physik, Universität Mainz, Mainz; Germany

98 School of Physics and Astronomy, University of Manchester, Manchester; United Kingdom

99 CPPM, Aix-Marseille Université, CNRS/IN2P3, Marseille; France

100 Department of Physics, University of Massachusetts, Amherst MA; United States of America

101 Department of Physics, McGill University, Montreal QC; Canada

102 School of Physics, University of Melbourne, Victoria; Australia

103 Department of Physics, University of Michigan, Ann Arbor MI; United States of America

104 Department of Physics and Astronomy, Michigan State University, East Lansing MI; United States of America

105 B.I. Stepanov Institute of Physics, National Academy of Sciences of Belarus, Minsk; Belarus

106 Research Institute for Nuclear Problems of Byelorussian State University, Minsk; Belarus

107 Group of Particle Physics, University of Montreal, Montreal QC; Canada

108 P.N. Lebedev Physical Institute of the Russian Academy of Sciences, Moscow; Russia

109 National Research Nuclear University MEPhI, Moscow; Russia

110 D.V. Skobeltsyn Institute of Nuclear Physics, M.V. Lomonosov Moscow State University, Moscow; Russia

111 Fakultät für Physik, Ludwig-Maximilians-Universität München, München; Germany

112 Max-Planck-Institut für Physik (Werner-Heisenberg-Institut), München; Germany

113 Nagasaki Institute of Applied Science, Nagasaki; Japan

114 Graduate School of Science and Kobayashi-Maskawa Institute, Nagoya University, Nagoya; Japan

115 Department of Physics and Astronomy, University of New Mexico, Albuquerque NM; United States of America

116 Institute for Mathematics, Astrophysics and Particle Physics, Radboud University/Nikhef, Nijmegen; Netherlands

117 Nikhef National Institute for Subatomic Physics and University of Amsterdam, Amsterdam; Netherlands

118 Department of Physics, Northern Illinois University, DeKalb IL; United States of America

119 Budker Institute of Nuclear Physics and NSU ${ }^{(a)}, S B$ RAS, Novosibirsk; Novosibirsk State University Novosibirsk ${ }^{(b)}$; Russia

120 Institute for High Energy Physics of the National Research Centre Kurchatov Institute, Protvino; Russia

121 Institute for Theoretical and Experimental Physics named by A.I. Alikhanov of National Research Centre "Kurchatov Institute", Moscow; Russia

123 Department of Physics, New York University, New York NY; United States of America

124 Ochanomizu University, Otsuka, Bunkyo-ku, Tokyo; Japan

125 Ohio State University, Columbus OH; United States of America

126 Homer L. Dodge Department of Physics and Astronomy, University of Oklahoma, Norman OK; United States of America 
St. Petersburg; Russia

136 Department of Physics and Astronomy, University of Pittsburgh, Pittsburgh PA; United States of America

137 Laboratório de Instrumentação e Fúsica Experimental de Partículas - LIP ${ }^{(a)}$, Lisboa; Departamento de Física ${ }^{(b)}$, Faculdade de Ciências, Universidade de Lisboa, Lisboa; Departamento de Física ${ }^{(c)}$, Universidade de Coimbra, Coimbra; Centro de Física Nuclear da Universidade de Lisboa $^{(d)}$, Lisboa; Departamento de Física ${ }^{(e)}$, Universidade do Minho, Braga; Departamento de Física Teórica y del Cosmos ${ }^{(f)}$, Universidad de Granada, Granada (Spain); Dep Física and CEFITEC of Faculdade de Ciências e Tecnologia ${ }^{(g)}$, Universidade Nova de Lisboa, Caparica; Instituto Superior Técnico ${ }^{(h)}$, Universidade de Lisboa, Lisboa; Portugal

138 Institute of Physics of the Czech Academy of Sciences, Prague; Czech Republic

139 Czech Technical University in Prague, Prague; Czech Republic

140 Charles University, Faculty of Mathematics and Physics, Prague; Czech Republic

141 Particle Physics Department, Rutherford Appleton Laboratory, Didcot; United Kingdom

142 IRFU, CEA, Université Paris-Saclay, Gif-sur-Yvette; France

143 Santa Cruz Institute for Particle Physics, University of California Santa Cruz, Santa Cruz CA; United States of America

144 Departamento de Fúsica ${ }^{(a)}$, Pontificia Universidad Católica de Chile, Santiago; Millennium Institute for Subatomic physics at high energy frontier $(\text { SAPHIR })^{(b)}$, Santiago; Universidad Andres Bello $^{(d)}$, Department of Physics, Santiago; Instituto de Alta Investigación ${ }^{(e)}$, Universidad de Tarapacá, Arica; Departamento de Física ${ }^{(f)}$, Universidad Técnica Federico Santa María, Valparaíso; Chile

145 Universidade Federal de São João del Rei (UFSJ), São João del Rei; Brazil

146 Department of Physics, University of Washington, Seattle WA; United States of America

147 Department of Physics and Astronomy, University of Sheffield, Sheffield; United Kingdom

148 Department of Physics, Shinshu University, Nagano; Japan

149 Department Physik, Universität Siegen, Siegen; Germany

150 Department of Physics, Simon Fraser University, Burnaby BC; Canada

151 SLAC National Accelerator Laboratory, Stanford CA; United States of America

152 Department of Physics, Royal Institute of Technology, Stockholm; Sweden

153 Departments of Physics and Astronomy, Stony Brook University, Stony Brook NY; United States of America

154 Department of Physics and Astronomy, University of Sussex, Brighton; United Kingdom

155 School of Physics, University of Sydney, Sydney; Australia

156 Institute of Physics, Academia Sinica, Taipei; Taiwan

157 E. Andronikashvili Institute of Physics ${ }^{(a)}$, Iv. Javakhishvili Tbilisi State University, Tbilisi; High Energy Physics Institute ${ }^{(b)}$, Tbilisi State University, Tbilisi; Georgia

158 Department of Physics, Technion, Israel Institute of Technology, Haifa; Israel

159 Raymond and Beverly Sackler School of Physics and Astronomy, Tel Aviv University, Tel Aviv; Israel

160 Department of Physics, Aristotle University of Thessaloniki, Thessaloniki; Greece

161 International Center for Elementary Particle Physics and Department of Physics, University of Tokyo, Tokyo; Japan 
164 Department of Physics, University of Toronto, Toronto ON; Canada

165 TRIUMF $^{(a)}$, Vancouver BC; Department of Physics and Astronomy ${ }^{(b)}$, York University, Toronto ON; Canada

166 Division of Physics and Tomonaga Center for the History of the Universe, Faculty of Pure and Applied Sciences, University of Tsukuba, Tsukuba; Japan

167 Department of Physics and Astronomy, Tufts University, Medford MA; United States of America

168 Department of Physics and Astronomy, University of California Irvine, Irvine CA; United States of America

169 Department of Physics and Astronomy, University of Uppsala, Uppsala; Sweden

170 Department of Physics, University of Illinois, Urbana IL; United States of America

171 Instituto de Física Corpuscular (IFIC), Centro Mixto Universidad de Valencia - CSIC, Valencia; Spain

172 Department of Physics, University of British Columbia, Vancouver BC; Canada

173 Department of Physics and Astronomy, University of Victoria, Victoria BC; Canada

174 Fakultät für Physik und Astronomie, Julius-Maximilians-Universität Würzburg, Würzburg; Germany

175 Department of Physics, University of Warwick, Coventry; United Kingdom

176 Waseda University, Tokyo; Japan

177 Department of Particle Physics and Astrophysics, Weizmann Institute of Science, Rehovot; Israel

178 Department of Physics, University of Wisconsin, Madison WI; United States of America

179 Fakultät für Mathematik und Naturwissenschaften, Fachgruppe Physik, Bergische Universität Wuppertal, Wuppertal; Germany

180 Department of Physics, Yale University, New Haven CT; United States of America

a Also at Borough of Manhattan Community College, City University of New York, New York NY; United States of America

${ }^{b}$ Also at Bruno Kessler Foundation, Trento; Italy

${ }^{c}$ Also at Center for High Energy Physics, Peking University; China

d Also at Centro Studi e Ricerche Enrico Fermi; Italy

e Also at CERN, Geneva; Switzerland

${ }^{f}$ Also at CPPM, Aix-Marseille Université, CNRS/IN2P3, Marseille; France

$g$ Also at Département de Physique Nucléaire et Corpusculaire, Université de Genève, Genève; Switzerland

${ }^{h}$ Also at Departament de Fisica de la Universitat Autonoma de Barcelona, Barcelona; Spain

${ }^{i}$ Also at Department of Financial and Management Engineering, University of the Aegean, Chios; Greece

${ }^{j}$ Also at Department of Physics and Astronomy, Michigan State University, East Lansing MI; United States of America

${ }^{k}$ Also at Department of Physics and Astronomy, University of Louisville, Louisville, KY; United States of America

${ }^{l}$ Also at Department of Physics, Ben Gurion University of the Negev, Beer Sheva; Israel

$m$ Also at Department of Physics, California State University, East Bay; United States of America

$n$ Also at Department of Physics, California State University, Fresno; United States of America

o Also at Department of Physics, California State University, Sacramento; United States of America

${ }^{p}$ Also at Department of Physics, King's College London, London; United Kingdom

${ }^{q}$ Also at Department of Physics, St. Petersburg State Polytechnical University, St. Petersburg; Russia

${ }^{r}$ Also at Department of Physics, University of Fribourg, Fribourg; Switzerland

$s$ Also at Faculty of Physics, M.V. Lomonosov Moscow State University, Moscow; Russia

${ }^{t}$ Also at Faculty of Physics, Sofia University, 'St. Kliment Ohridski', Sofia; Bulgaria

${ }^{u}$ Also at Giresun University, Faculty of Engineering, Giresun; Turkey 
$v$ Also at Graduate School of Science, Osaka University, Osaka; Japan

w Also at Hellenic Open University, Patras; Greece

$x$ Also at Institucio Catalana de Recerca i Estudis Avancats, ICREA, Barcelona; Spain

$y \quad$ Also at Institut für Experimentalphysik, Universität Hamburg, Hamburg; Germany

$z$ Also at Institute for Particle and Nuclear Physics, Wigner Research Centre for Physics, Budapest; Hungary

aa Also at Institute of Particle Physics (IPP); Canada

ab Also at Institute of Physics, Azerbaijan Academy of Sciences, Baku; Azerbaijan

ac Also at Institute of Theoretical Physics, Ilia State University, Tbilisi; Georgia

ad Also at Instituto de Fisica Teorica, IFT-UAM/CSIC, Madrid; Spain

ae Also at Istanbul University, Dept. of Physics, Istanbul; Turkey

af Also at Joint Institute for Nuclear Research, Dubna; Russia

ag Also at Moscow Institute of Physics and Technology State University, Dolgoprudny; Russia

ah Also at National Research Nuclear University MEPhI, Moscow; Russia

ai Also at Physikalisches Institut, Albert-Ludwigs-Universität Freiburg, Freiburg; Germany

aj Also at The City College of New York, New York NY; United States of America

ak Also at TRIUMF, Vancouver BC; Canada

al Also at Universita di Napoli Parthenope, Napoli; Italy

am Also at University of Chinese Academy of Sciences (UCAS), Beijing; China

an Also at Yeditepe University, Physics Department, Istanbul; Turkey

* Deceased 